\title{
MICROLOCALIZATION OF RATIONAL CHEREDNIK ALGEBRAS
}

\author{
MASAKI KASHIWARA AND RAPHAËL ROUQUIER
}

\begin{abstract}
We construct a microlocalization of the rational Cherednik algebras $H$ of type $S_{n}$. This is achieved by a quantization of the Hilbert scheme Hilb $\mathbb{C}^{2}$ of $n$ points in $\mathbb{C}^{2}$. We then prove the equivalence of the category of $H$-modules and the one of modules over its microlocalization under certain conditions on the parameter.
\end{abstract}

\section{Contents}

1. Introduction 1

2. F-actions on W-algebras 4

2.1. Notations 4

2.2. W-algebras 4

2.3. F-actions 6

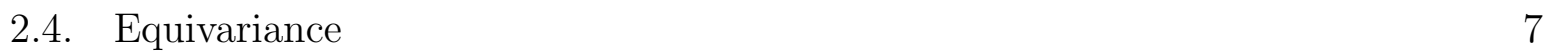

2.5. Symplectic reduction 9

2.6. $\mathscr{W}$-affinity 9

3. Rational Cherednik algebras and $\mathscr{D}$-modules 14

3.1. Definitions, notations and recollections 14

3.2. Construction of some $\mathscr{D}$-modules 14

3.3. Spherical constructions and shift $\quad 17$

4. Cherednik algebras and Hilbert schemes 25

4.1. Geometry of the Hilbert scheme 25

4.2. W-algebras on the Hilbert scheme 27

4.3. Affinity of $\mathscr{A}_{c} \quad 29$

4.4. W-algebras as fractions of $e H_{c} e \quad 32$

4.5. Rank 2 case $\quad 34$

References $\quad 35$

\section{INTRODUCTION}

Let us recall that Hilb $^{n} \mathbb{C}^{2}$, the Hilbert scheme of $n$ points in $\mathbb{C}^{2}$, is a symplectic (in particular crepant) resolution of $\mathbb{C}^{2 n} / S_{n}=S^{n} \mathbb{C}^{2}$. On the other hand, the orbifold $\left[\mathbb{C}^{2 n} / S_{n}\right]$ (or the corresponding algebra $\left.\mathbb{C}\left[\mathbb{C}^{2 n}\right] \rtimes S_{n}\right)$ is a non-commutative crepant resolution of $\mathbb{C}^{2 n} / S_{n}$.

Date: May 8, 2007.

1991 Mathematics Subject Classification. Primary:16G89,53D55; Secondary:14C05.

Key words and phrases. Rational Cherednik algebra, Hilbert scheme, microlocalization.

The first author is partially supported by Grant-in-Aid for Scientific Research (B) 18340007, Japan Society for the Promotion of Science. 
There is an equivalence between derived categories of coherent sheaves on $\operatorname{Hilb}^{n} \mathbb{C}^{2}$ and finitely generated modules over $\mathbb{C}\left[\mathbb{C}^{2 n}\right] \rtimes S_{n}$ (McKay's correspondence, cf. [11]).

The rational Cherednik algebra $H_{c}$ associated with $S_{n}$ is a one-parameter quantization of $\mathbb{C}\left[\mathbb{C}^{2 n}\right] \rtimes S_{n}$. We construct a one-parameter quantization $\widetilde{\mathscr{A}}_{c}$ of $\mathscr{O}_{\mathrm{Hilb}^{n} \mathbb{C}^{2}}$ and an equivalence of categories between a certain category of $\widetilde{\mathscr{A}_{c}}$-modules (good modules with $F$-action) and the category of finitely generated $H_{c}$-modules (under certain conditions on the parameter $c$ ). Note that this is an equivalence of abelian categories, while the non-quantized McKay's correspondence is only an equivalence of derived categories.

The quantization $\widetilde{\mathscr{A}_{c}}$ is a sheaf over $\mathrm{Hilb}^{n} \mathbb{C}^{2}$. Locally on an open subset isomorphic to $T^{*} U$, it is isomorphic to the sheaf of micro-differential operators $\mathscr{W}$ with a homogenizing parameter $\hbar$.

Note that our construction is an analog of the Beilinson-Bernstein localization Theorem for universal enveloping algebras upon flag varieties:

\begin{tabular}{|l|l|}
\hline nilpotent cone $\mathscr{N}$ & $\mathbb{C}^{2 n} / S_{n}$ \\
enveloping algebra quotients $U_{\lambda}(\mathfrak{g})$ & $H_{c}$ \\
$T^{*}(G / B)$ & $\operatorname{Hilb}^{n} \mathbb{C}^{2}$ \\
$\mathscr{D}_{G / B . \lambda}$ & $\widetilde{\mathscr{A}_{c}}$ \\
\hline
\end{tabular}
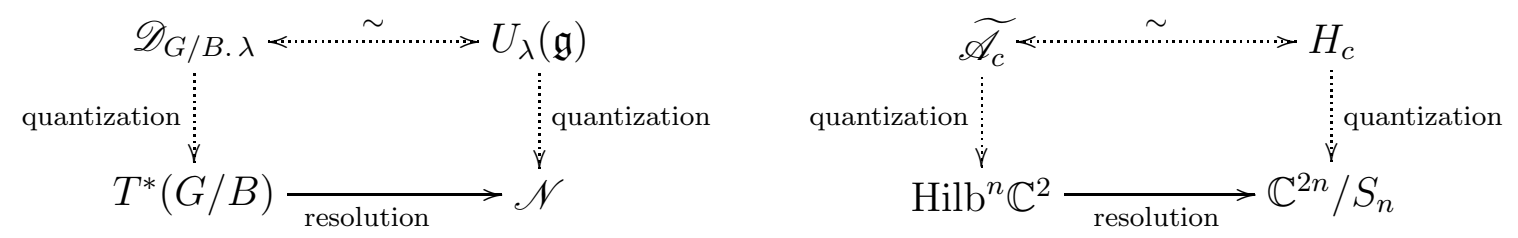

Let us mention that our constructions give rise to the spherical subalgebra $e H_{c} e$ of $H_{c}$ and under certain assumptions on $c$ the two algebras are Morita equivalent. It would be interesting to quantize directly the Procesi bundle to obtain $H_{c}$.

Let us now describe some earlier results related to our work. An important achievement of Etingof and Ginzburg [6] and of Gan and Ginzburg [7] is a construction of a deformation of the Harish-Chandra morphism for $\mathrm{GL}_{n}(\mathbb{C})$, providing a construction of the spherical subalgebra $e H_{c} e$ of $H_{c}$ as a quantum Hamiltonian reduction. This provides a quantization of the Calogero-Moser space, which is itself obtained by classical Hamiltonian reduction (Kazhdan, Kostant and Sternberg [21]).

Gordon and Stafford [8,9] construct a one-parameter family of graded $(\mathbb{Z})$-algebras $\mathcal{B}_{c}$ that quantize (a graded $(\mathbb{Z})$-algebra Morita-equivalent to) the homogeneous coordinate ring of $\mathrm{Hilb}^{n} \mathbb{C}^{2}$.

In positive characteristic, Bezrukavnikov, Finkelberg and Ginzburg [4] construct a sheaf of Azumaya algebras on the Hilbert scheme whose algebra of global sections is isomorphic to $H_{c}$ and obtain an equivalence of derived categories between modules over that Azumaya algebra and representations of $H_{c}$.

Let us explain the type of sheaf of algebras used to quantize $\mathrm{Hilb}^{n} \mathbb{C}^{2}$. On a complex contact manifold, Kashiwara [16] constructed the stack $\mathcal{E}$ of microdifferential operators. Locally, a model for a contact manifold is the projectivized cotangent bundle $P^{*} X$ and 
the stack $\mathcal{E}$ comes from the sheaf $\mathcal{E}_{X}$ of microdifferential operators of Sato, Kawai and Kashiwara.

On a symplectic variety, Kontsevich [22] and Polesello-Schapira [24] defined a stack $\mathscr{W}$ of microdifferential operators with a homogenizing parameter $\hbar$ (making all objects modules over $\mathbb{C}((\hbar)))$. Locally, a model is $T^{*} X$ and $\mathscr{W}$ comes from microdifferential operators on $P^{*}(X \times \mathbb{C})$ which do not depend on the extra variable.

For applications to representation theory, these constructions are unsatisfactory:

- the first construction "forgets about the zero-section"

- the second construction gives "too large" objects (defined over $\mathbb{C}((\hbar))$ instead of $\mathbb{C})$.

To overcome these difficulties, we consider here symplectic manifolds $X$ with a $\mathbb{C}^{\times}$action that stabilizes $\mathbb{C} \omega_{X}$ with a positive weight. We consider the case where the stack $\mathscr{W}$ comes from a sheaf of algebras together with a compatible action of $\mathbb{C}^{\times}$and study the corresponding structure, a "W-algebra with F-action". The category of its modules is defined over $\mathbb{C}$, as the $\mathrm{F}$-action induces a $\mathbb{C}^{\times}$- action on $\mathbb{C}((\hbar))$ whose invariant field is $\mathbb{C}$.

Let us now describe the structure of the paper.

In the first part of this paper $(\S 2)$, we study a general setting for the quantization of symplectic manifolds $X$ with a $\mathbb{C}^{\times}$-action that stabilizes $\mathbb{C} \omega_{X}$ with a positive weight. We first review the theory of $\mathrm{W}$-algebras on symplectic manifolds $(\S 2.2)$. In $\S 2.3$, we introduce the notion of "W-algebra with F-action". An important point of this construction is that the category of $\mathscr{W}$-modules with $\mathrm{F}$-action on a cotangent bundle (for the canonical structure) is equivalent to the category of modules over the sheaf $\mathscr{D}$ of differential operators. We adapt in $\S 2.4$ the study of equivariance and its twisted version for the action of a complex Lie group and we explain how to construct $\mathrm{W}$-algebras with F-action by symplectic reduction in $\S 2.5$. Finally, in $\S 2.6$ we provide sufficient conditions to ensure $\mathscr{W}$-affinity (a counterpart of Beilinson-Bernstein's result for $\mathscr{D}$-modules).

Section 3 is devoted to the construction of $\mathscr{D}$-modules with an action of the rational Cherednik algebra $H_{c}$ of type $A_{n-1}$ or of its spherical subalgebra $e H_{c} e$. This is related to the constructions of $[4,7]$. Let $V=\mathbb{C}^{n}$ and $\mathfrak{g}=\mathfrak{g l}_{n}(\mathbb{C})$. We construct $(\S 3.2)$ a quasi-coherent $\mathscr{D}_{\mathfrak{g} \times V}$-module $\mathscr{M}_{c}$ together with an action of $H_{c}$, building on the explicit description of the $\mathscr{D}$-module arising in Springer's correspondence given in [14]. We con-

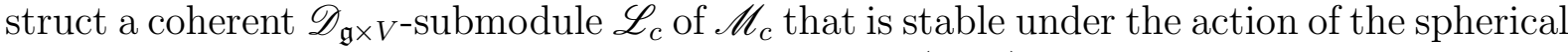
subalgebra of $H_{c}$ and we construct a shift operator ( $\left.\S 3.3\right)$. This is achieved by reduction to rank 2.

In $\S 4$ we construct a $\mathrm{W}$-algebra with F-action on Hilb $\mathbb{C}^{2}$ by symplectic reduction from the previous constructions. After recalling some properties of $\mathrm{Hilb}^{n} \mathbb{C}^{2}$ in $\S 4$.1, we construct in $\S 4.2$ a W-algebra $\widetilde{\mathscr{A}}_{c}$ on $\mathrm{Hilb}^{n} \mathbb{C}^{2}$ by symplectic reduction of $\mathscr{L}_{c}$ for the action of $\mathrm{GL}_{n}(\mathbb{C})$. In $\S 4.3$, we present our main results: $\widetilde{\mathscr{A}}_{c}$-affinity of $\mathrm{Hilb}^{n} \mathbb{C}^{2}$, an isomorphism between global sections of $\widetilde{\mathscr{A}}_{c}$ and the spherical algebra and an equivalence between the category of good $\widetilde{\mathscr{A}_{c}}$-modules with $F$-action and the one of finitely generated modules over the spherical algebra. We also describe similar results for $H_{c}$. So, we have obtained a microlocalization of the rational Cherednik algebras: we have constructed a $\mathrm{W}$-algebra with F-action over the Hilbert scheme whose algebra of global sections is isomorphic 
to $H_{c}$ and whose modules are equivalent to representations of $H_{c}$. Those results are obtained under certain assumptions on $c$. We explain in $\S 4.4$ how to view sections of our $\mathrm{W}$-algebras over open subsets of the Hilbert schemes as appropriate fractions in the Cherednik algebra. Finally, we describe explicitly the constructions for $n=2$ in $\S 4.5$.

We thank Pierre Schapira for some useful discussions. The first author thanks Shigeru Mukai for his comments on Hilbert schemes. The second author thanks Research Institute for Mathematical Sciences, Kyoto University, for its hospitality.

\section{F-ACTIONS ON W-ALGEBRAS}

2.1. Notations. By a manifold $M$, we mean a complex manifold, equipped with the classical topology and $\mathscr{O}_{M}$ is the sheaf of holomorphic functions. We denote by $\mathscr{D}_{M}$ the sheaf of differential operators with holomorphic coefficients and by $\mathcal{E}_{M}$ the sheaf of formal micro-differential operators on the cotangent bundle $T^{*} M$.

We denote by $\mathbb{G}_{\mathrm{m}}$ the multiplicative group $\mathbb{C}^{\times}$.

Given a ring $A$, we denote by $\operatorname{Mod}_{\text {coh }}(A)$ the category of coherent left $A$-modules.

2.2. $\mathbf{W}$-algebras. We shall review some results on $\mathrm{W}$-algebras. We refer the reader to [24] (where the convergent version is studied, while we use the simpler formal version).

2.2.1. Let $\mathbf{k}=\mathbb{C}((\hbar))$ be the field of formal Laurent series in an indeterminate $\hbar$ and let $\mathbf{k}(0)=\mathbb{C}[[\hbar]]$. Given $m \in \mathbb{Z}$, we define $\mathscr{W}_{T^{*} \mathbb{C}^{n}}(m)$ as the sheaf of formal series $\sum_{k \geqslant-m} \hbar^{k} a_{k}$ $\left(a_{k} \in \mathscr{O}_{T^{*} \mathbb{C}^{n}}\right)$ on the cotangent bundle $T^{*} \mathbb{C}^{n}$ of $\mathbb{C}^{n}$ and we set $\mathscr{W}_{T^{*} \mathbb{C}^{n}}=\cup_{m} \mathscr{W}_{T^{*} \mathbb{C}^{n}}(m)$. Then, $\mathscr{W}_{T^{*} \mathbb{C}^{n}}$ has a structure of $\mathbf{k}$-algebra given by

$$
a \circ b=\sum_{\alpha \in \mathbb{Z}_{\geqslant 0}^{n}} \hbar^{|\alpha|} \frac{1}{\alpha !} \partial_{\xi}^{\alpha} a \cdot \partial_{x}^{\alpha} b .
$$

We have a ring homomorphism $\mathscr{D}_{\mathbb{C}^{n}}\left(\mathbb{C}^{n}\right) \rightarrow \mathscr{W}_{T^{*} \mathbb{C}^{n}}\left(T^{*} \mathbb{C}^{n}\right)$ given by $x_{i} \mapsto x_{i}, \frac{\partial}{\partial x_{i}} \mapsto \hbar^{-1} \xi_{i}$.

2.2.2. Let $X$ be a complex symplectic manifold with symplectic form $\omega_{X}$. We denote by $X^{\text {opp }}$ the symplectic manifold $X$ with symplectic form $-\omega_{X}$.

A W-algebra is a k-algebra $\mathscr{W}$ on $X$ such that for any point $x \in X$, there are an open neighbourhood $U$ of $x$, a symplectic map $f: U \rightarrow T^{*} \mathbb{C}^{n}$ and a k-algebra isomorphism $g:\left.\mathscr{W}\right|_{U} \stackrel{\sim}{\longrightarrow} f^{-1} \mathscr{W}_{T^{*} \mathbb{C}^{n}}$.

A $\mathrm{W}$-algebra $\mathscr{W}$ satisfies the following properties.

(i) The algebra $\mathscr{W}$ is a coherent and noetherian algebra.

(ii) $\mathscr{W}$ contains a canonical subalgebra $\mathscr{W}(0)$ which is locally isomorphic to $\mathscr{W}_{T^{*} \mathbb{C}^{n}}(0)$ (via the maps $g$ ). We set $\mathscr{W}(m)=\hbar^{-m} \mathscr{W}(0)$.

(iii) We have a canonical $\mathbb{C}$-algebra isomorphism $\mathscr{W}(0) / \mathscr{W}(-1) \stackrel{\sim}{\sim} \mathscr{O}_{X}$ (coming from the canonical isomorphism via the maps $g$ ). The corresponding morphism $\sigma_{m}: \mathscr{W}(m) \rightarrow$ $\hbar^{-m} \mathscr{O}_{X}$ is called the symbol map.

(iv) We have

$$
\sigma_{0}\left(\hbar^{-1}[a, b]\right)=\left\{\sigma_{0}(a), \sigma_{0}(b)\right\}
$$

for any $a, b \in \mathscr{W}(0)$. Here $\{\bullet, \bullet\}$ is the Poisson bracket.

(v) The canonical map $\mathscr{W}(0) \rightarrow \lim _{m \rightarrow \infty} \mathscr{W}(0) / \mathscr{W}(-m)$ is an isomorphism.

(vi) A section $a$ of $\mathscr{W}(0)$ is invertible in $\mathscr{W}(0)$ if and only if $\sigma_{0}(a)$ is invertible in $\mathscr{O}_{X}$. 
(vii) Given $\phi$ a k-algebra automorphism of $\mathscr{W}$, we can find locally an invertible section $a$ of $\mathscr{W}(0)$ such that $\phi=\operatorname{Ad}(a)$. Moreover $a$ is unique up to a scalar multiple. In other words, we have canonical isomorphisms

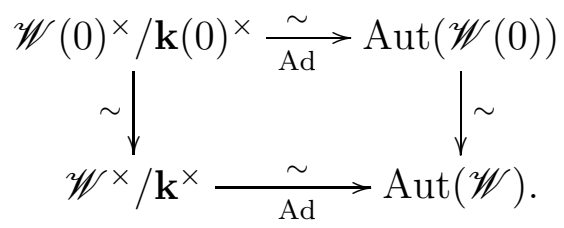

(viii) Let $v$ be a $\mathbf{k}$-linear filtration-preserving derivation of $\mathscr{W}$. Then there exists locally a section $a$ of $\mathscr{W}(1)$ such that $v=\operatorname{ad}(a)$. Moreover $a$ is unique up to a scalar. In other words, we have an isomorphism

$$
\mathscr{W}(1) / \hbar^{-1} \mathbf{k}(0) \underset{\text { ad }}{\sim} \operatorname{Der}_{\text {filtered }}(\mathscr{W}) .
$$

(ix) If $\mathscr{W}$ is a $\mathrm{W}$-algebra, then its opposite ring $\mathscr{W}^{\text {opp }}$ is a $\mathrm{W}$-algebra on $X^{\text {opp }}$.

Conjecturally, (iii), (iv) and (v) characterize $\mathscr{W}(0)$.

Note that two W-algebras on $X$ are locally isomorphic.

2.2.3. Assume there exist $a_{i}, b_{i} \in \mathscr{W}(0)(i=1, \ldots, n)$ such that $\left[a_{i}, a_{j}\right]=\left[b_{i}, b_{j}\right]=0$ and $\left[b_{i}, a_{j}\right]=\hbar \delta_{i j}$. They induce a symplectic map

$$
f=\left(\sigma_{0}\left(a_{1}\right), \ldots, \sigma_{0}\left(a_{n}\right) ; \sigma_{0}\left(b_{1}\right), \ldots, \sigma_{0}\left(b_{n}\right)\right): X \rightarrow T^{*} \mathbb{C}^{n} .
$$

Then, there exists a unique isomorphism

$$
\mathscr{W} \stackrel{\sim}{\longrightarrow} f^{-1} \mathscr{W}_{T^{*} \mathbb{C}^{n}}, a_{i} \mapsto x_{i}, b_{i} \mapsto \xi_{i}
$$

We call $\left(a_{1}, \ldots, a_{n} ; b_{1}, \ldots, b_{n}\right)$ quantized symplectic coordinates of $\mathscr{W}$.

Let $M$ be a complex manifold $M$ and $\pi_{M}: T^{*} M \rightarrow M$ the projection. We can associate canonically a W-algebra $\mathscr{W}_{T^{*} M}$ with a morphism $\pi_{M}^{-1} \mathscr{D}_{M} \rightarrow \mathscr{W}_{T^{*} M}$ such that

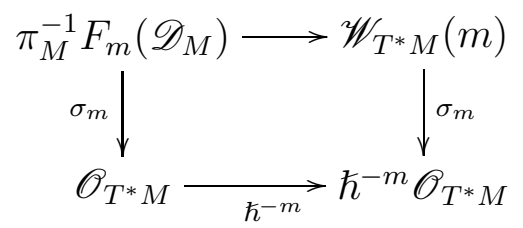

commutes. Here, $F\left(\mathscr{D}_{M}\right)$ is the order filtration of $\mathscr{D}_{M}$. Note that $\pi_{M}^{-1} \mathscr{D}_{M} \rightarrow \mathscr{W}_{T^{*} M}$ decomposes into $\pi_{M}^{-1} \mathscr{D}_{M} \rightarrow \mathcal{E}_{M} \rightarrow \mathscr{W}_{T^{*} M}$. The ring $\mathscr{W}_{T^{*} M}$ is flat over $\pi_{M}^{-1} \mathscr{D}_{M}$ and faithfully flat over $\mathcal{E}_{M}$. In particular, for a coherent $\mathscr{D}_{M}$-module $\mathscr{M}$, the characteristic variety $\operatorname{Ch}(\mathscr{M})$ coincides with $\operatorname{Supp}\left(\mathscr{W}_{T^{*} M} \otimes_{\pi_{M}^{-1} \mathscr{D}_{M}} \pi_{M}^{-1} \mathscr{M}\right)$.

Let $X$ and $Y$ be two symplectic manifolds. The product $X \times Y$ is also a symplectic manifold. For a $\mathrm{W}$-algebra $\mathscr{W}_{X}$ on $X$ and a $\mathrm{W}$-algebra $\mathscr{W}_{Y}$ on $Y$, there is a $\mathrm{W}$-algebra $\mathscr{W}_{X} \otimes \mathscr{W}_{Y}$ on $X \times Y$. Letting $p_{1}: X \times Y \rightarrow X$ and $p_{2}: X \times Y \rightarrow Y$ be the projections, $\mathscr{W}_{X} \otimes \mathscr{W}_{Y}$ contains $p_{1}^{-1} \mathscr{W}_{X} \otimes_{\mathbf{k}} p_{2}^{-1} \mathscr{W}_{Y}$ as a $\mathbf{k}$-subalgebra, and is faithfully flat over it.

For a $\mathscr{W}$-module $\mathscr{M}$, a $\mathscr{W}(0)$-lattice is a coherent $\mathscr{W}(0)$-submodule $\mathscr{N}$ of $\mathscr{M}$ such that the canonical map $\mathscr{W} \otimes_{\mathscr{W}(0)} \mathscr{N} \rightarrow \mathscr{M}$ is an isomorphism.

We say that a $\mathscr{W}$-module $\mathscr{M}$ is good if for any relatively compact open subset $U$ of $X$, there exists a coherent $\left.\mathscr{W}(0)\right|_{U}$-lattice of $\left.\mathscr{M}\right|_{U}$. The full subcategory of good $\mathscr{W}$-modules is an abelian subcategory of the category of $\mathscr{W}$-modules. 
The following fact will be used in this paper (see [20, Theorem 1.2.2], where the convergent version is proved).

Lemma 2.1. Let $r$ be an integer and let $\mathscr{M}$ be a coherent $\mathscr{W}$-module such that $\mathscr{E} x t_{\mathscr{W}}^{j}(\mathscr{M}, \mathscr{W})=$ 0 for any $j>r$. Then $\mathscr{H}_{S}^{j}(\mathscr{M})=0$ for any closed analytic subset $S$ and any $j<$ $\operatorname{codim} S-r$.

Let $\overline{\mathbf{k}}:=\cup_{n>0} \mathbb{C}\left(\left(\hbar^{1 / n}\right)\right)$ be an algebraic closure of $\mathbf{k}$. We will sometimes need to replace $\mathscr{W}$ with $\mathbf{k}^{\prime} \otimes_{\mathbf{k}} \mathscr{W}$ for some field $\mathbf{k}^{\prime}$ with $\mathbf{k} \subset \mathbf{k}^{\prime} \subset \overline{\mathbf{k}}$.

\subsection{F-actions.}

2.3.1. Let $X$ be a symplectic manifold. Consider an action of $\mathbb{G}_{\mathrm{m}}$ on $X$, viewed as a manifold: $\mathbb{C}^{\times} \ni t \mapsto T_{t} \in \operatorname{Aut}(X)$. We assume $\mathbb{G}_{\mathrm{m}}$ stabilizes the line $\mathbb{C} \omega_{X} \subset H^{0}\left(X, \Omega_{X}^{2}\right)$ with a positive weight $m$, i.e., $T_{t}^{*} \omega_{X}=t^{m} \omega_{X}$ for all $t \in \mathbb{C}^{\times}$.

We denote by $v$ the vector field given by the $\mathbb{G}_{\mathrm{m}}$-action: $v(a)(x)=\left.\frac{d}{d t} a\left(T_{t}(x)\right)\right|_{t=1}$. The Poisson bracket $\{\bullet, \bullet\}$ is homogeneous of degree $-m$ :

$$
T_{t}^{*}\{a, b\}=t^{-m}\left\{T_{t}^{*} a, T_{t}^{*} b\right\} \text { and } v\{a, b\}=\{v(a), b\}+\{a, v(b)\}-m\{a, b\} \text { for } a, b \in \mathscr{O}_{X} .
$$

Let $\mathscr{W}$ be a $\mathrm{W}$-algebra.

Definition 2.2. An F-action with exponent $m$ on $\mathscr{W}$ is an action of $\mathbb{G}_{\mathrm{m}}$ on the $\mathbb{C}$ algebra $\mathscr{W}, \mathcal{F}_{t}: T_{t}^{-1} \mathscr{W} \stackrel{\sim}{\longrightarrow}$ for $t \in \mathbb{C}^{\times}$, such that $\mathcal{F}_{t}(\hbar)=t^{m} \hbar$ and $\mathcal{F}_{t}(a)$ depends holomorphically on $t$ for any $a \in \mathscr{W}$.

Let us fix an F-action with exponent $m$ on $\mathscr{W}$. The $\mathbb{G}_{\mathrm{m}}$-action induces an orderpreserving derivation $v_{F}$ of $\mathscr{W}$ given by $v_{F}(a)=\left.\frac{d}{d t} \mathcal{F}_{t}(a)\right|_{t=1}$. It satisfies the following properties:

$$
\begin{aligned}
& v_{F}(\hbar)=m \hbar, \\
& \sigma_{0}\left(v_{F}(a)\right)=v\left(\sigma_{0}(a)\right) \quad \text { for } a \in \mathscr{W}(0) .
\end{aligned}
$$

Remark 2.3. Here, $\mathrm{F}$ stands for "Frobenius". Note that $v_{F}$ determines the F-action on $\mathscr{W}$. However, for a given $v_{F}$ satisfying (2.1), we cannot always find an F-action on $\mathscr{W}$.

The action of $\mathbb{G}_{\mathrm{m}}$ on $\mathscr{W}$ extends to an action on $\mathscr{W}\left[\hbar^{1 / m}\right]=\mathbf{k}\left(\hbar^{1 / m}\right) \otimes_{\mathbf{k}} \mathscr{W}$ given by $\mathcal{F}_{t}\left(\hbar^{1 / m}\right)=t \hbar^{1 / m}$.

Definition 2.4. A $\mathscr{W}\left[\hbar^{1 / m}\right]$-module with an F-action (or simply a $\left(\mathscr{W}\left[\hbar^{1 / m}\right], \mathcal{F}\right)$-module) is a $\mathbb{G}_{\mathrm{m}}$-equivariant $\mathscr{W}\left[\hbar^{1 / m}\right]$-module: we have isomorphisms $\mathcal{F}_{t}: T_{t}^{-1} \mathscr{M} \stackrel{\mathscr{M}}{\sim}$ for $t \in$ $\mathbb{C}^{\times}$and we assume that

(a) $\mathcal{F}_{t}(u)$ depends holomorphically on $t$ for any $u \in \mathscr{M}$ (i.e., there exist locally finitely many $u_{i}$ such that $\mathcal{F}_{t}(u)=\sum_{i} a_{i}(t) u_{i}$ where $a_{i}(t) \in \mathscr{W}\left[\hbar^{1 / m}\right]$ depends holomorphically on $t)$,

(b) $\mathcal{F}_{t}(a u)=\mathcal{F}_{t}(a) \mathcal{F}_{t}(u)$ for $a \in \mathscr{W}\left[\hbar^{1 / m}\right], u \in \mathscr{M}$,

(c) $\mathcal{F}_{t} \circ \mathcal{F}_{t^{\prime}}=\mathcal{F}_{t t^{\prime}}$ for $t, t^{\prime} \in \mathbb{C}^{\times}$.

We denote by $\operatorname{Mod}_{F}\left(\mathscr{W}\left[\hbar^{1 / m}\right]\right)$ the category of $\left(\mathscr{W}\left[\hbar^{1 / m}\right], \mathcal{F}\right)$-modules: morphisms are morphisms of $\mathscr{W}\left[\hbar^{1 / m}\right]$-modules compatible with the $\mathbb{G}_{\mathrm{m}}$-action. We denote by $\operatorname{Mod}_{F}^{\text {good }}\left(\mathscr{W}\left[\hbar^{1 / m}\right]\right)$ its full subcategory of good $\left(\mathscr{W}\left[\hbar^{1 / m}\right], \mathcal{F}\right)$-modules. These are $\mathbb{C}$-linear abelian categories. 
Note that if there is a relatively compact open subset $U$ of $X$ such that $\mathbb{C}^{\times} \cdot U=X$, then a good $\left(\mathscr{W}\left[\hbar^{1 / m}\right], \mathcal{F}\right)$-module admits a coherent $\left(\mathscr{W}(0)\left[\hbar^{1 / m}\right], \mathcal{F}\right)$-lattice.

Assume $X=\{\mathrm{pt}\}$, so that $\mathscr{W}=\mathbf{k}$. We have an equivalence $\operatorname{Mod}_{F}\left(\mathscr{W}\left[\hbar^{1 / m}\right]\right) \stackrel{\sim}{\longrightarrow} \operatorname{Mod}(\mathbb{C})$, $\mathscr{M} \mapsto \mathscr{M}^{\mathbb{G}_{\mathrm{m}}}$, with quasi-inverse $V \mapsto \mathbb{C}\left(\left(\hbar^{1 / m}\right)\right) \otimes_{\mathbb{C}} V$.

Remark 2.5. Kontsevich and Kaledin [15] have also studied quantization for a symplectic variety with a $\mathbb{G}_{\mathrm{m}}$-action that stabilizes $\mathbb{C} \omega_{X}$ with a positive weight.

2.3.2. Let $\mathscr{W}$ be a $\mathrm{W}$-algebra with an F-action with exponent $m$. Let $n$ be a positive integer and consider the restriction of the F-action via $\mathbb{G}_{\mathrm{m}} \rightarrow \mathbb{G}_{\mathrm{m}}, t \mapsto t^{n}$ : we have a new action given by $T_{t}^{\prime}=T_{t^{n}}$ and $\mathcal{F}_{t}^{\prime}=\mathcal{F}_{t^{n}}$. This defines an F-action on $\mathscr{W}$ with exponent $m n$. Then, we have quasi-inverse equivalences of categories

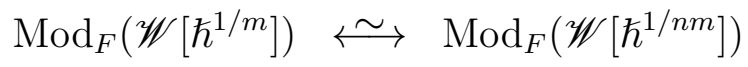

$$
\begin{aligned}
& \mathscr{M} \mapsto \mathscr{W}\left[\hbar^{1 / n m}\right] \otimes_{\mathscr{W}\left[\hbar^{1 / m}\right]} \mathscr{M} \\
& \left\{s \in \mathscr{N} ; \mathcal{F}_{\zeta}^{\prime}(s)=s \text { for any } \zeta \in \mathbb{C} \text { with } \zeta^{n}=1\right\} \quad \longleftrightarrow \quad \mathscr{N}
\end{aligned}
$$

Remark 2.6. The equivalence above shows the category depends only on the 1-parameter subgroup of $\operatorname{Aut}(X, \mathscr{W})$ given by the $\mathbb{G}_{\mathrm{m}}$-action.

Let $\hat{\mathbb{G}}_{\mathrm{m}}={\underset{n}{n}}_{\lim } \mathbb{G}_{\mathrm{m}}$, where the limit is taken over maps $f_{n, n^{\prime}}: \mathbb{G}_{\mathrm{m}} \rightarrow \mathbb{G}_{\mathrm{m}}, t \mapsto t^{n / n^{\prime}}$ for positive integers $n, n^{\prime}$ with $n^{\prime} \mid n$. This is a pro-algebraic group (some sort of universal covering group of $\left.\mathbb{G}_{\mathrm{m}}\right)$. In terms of functions, we have $\hat{\mathbb{G}}_{\mathrm{m}}=\operatorname{Spec}\left(\bigoplus_{a \in \mathbb{Q}} \mathbb{C} t^{a}\right)$ with multiplication coming from the coproduct $t^{a} \mapsto t^{a} \otimes t^{a}$. Instead of considering $\mathbb{G}_{\mathrm{m}}$-actions as above, we could consider $\hat{\mathbb{G}}_{\mathrm{m}}$-actions on $X$ such that $T_{t}^{*} \omega_{X}=t \omega_{X}$. Although theoretically more satisfactory, this more complicated formulation is not used in the present paper.

\subsubsection{Let us now give two examples.}

Let $M$ be a manifold, $X=T^{*} M$ and $\mathscr{W}=\mathscr{W}_{T^{*} M}$. We consider the canonical $\mathbb{G}_{\mathrm{m}^{-}}$ action given by $T_{t}(x, \xi)=(x, t \xi)$. There is a unique $\mathrm{F}$-action with exponent 1 on $\mathscr{W}$ with $\left.\mathcal{F}\right|_{\mathscr{D}_{M}}=\mathrm{id}$. Then, for any $\mathbb{G}_{\mathrm{m}}$-invariant open subset $U$ of $X$, we have an equivalence

$$
\operatorname{Mod}_{F}^{\text {good }}\left(\left.\mathscr{W}\right|_{U}\right) \stackrel{\sim}{\longrightarrow} \operatorname{Mod}_{\text {good }}\left(\left.\mathcal{E}_{M}\right|_{U}\right), \mathscr{M} \mapsto \mathscr{M}^{\mathbb{G}_{\mathrm{m}}}
$$

In particular, we have an equivalence

$$
\operatorname{Mod}_{F}^{\text {good }}(\mathscr{W}) \stackrel{\sim}{\longrightarrow} \operatorname{Mod}_{\text {good }}\left(\mathscr{D}_{M}\right)
$$

Let $X=T^{*} \mathbb{C}^{n}$ and $\mathscr{W}=\mathscr{W}_{T^{*} \mathbb{C}^{n}}$. Fix $m>1$ and $l_{1}, \ldots, l_{n} \in\{1, \ldots, m-1\}$. We define a $\mathbb{G}_{\mathrm{m}}$-action by $T_{t}\left(\left(x_{i}\right),\left(\xi_{i}\right)\right)=\left(\left(t^{l_{i}} x_{i}\right),\left(t^{m-l_{i}} \xi_{i}\right)\right)$. Then $T_{t}^{*}\left(\omega_{X}\right)=t^{m} \omega_{X}$. We define an F-action on $\mathscr{W}$ with exponent $m$ by $\mathcal{F}_{t}\left(x_{i}\right)=t^{l_{i}} x_{i}, \mathcal{F}_{t}\left(\partial_{i}\right)=t^{-l_{i}} \partial_{i}$, and $\mathcal{F}_{t}(\hbar)=t^{m} \hbar$ (note that the relation $\left[\partial_{i}, x_{i}\right]=1$ is preserved by $\left.\mathcal{F}_{t}\right)$. Then,

$$
\operatorname{End}_{\operatorname{Mod}_{F}\left(\mathscr{W}\left[\hbar^{1 / m}\right]\right)}\left(\mathscr{W}\left[\hbar^{1 / m}\right]\right)^{\mathrm{opp}}=\mathbb{C}\left[\hbar^{-l_{i} / m} x_{i}, \hbar^{l_{i} / m} \partial_{i} ; i=1, \ldots, n\right] \subset \mathscr{W}\left[\hbar^{1 / m}\right],
$$

which is isomorphic to $\mathscr{D}\left(\mathbb{C}^{n}\right)$. Moreover, $\operatorname{Mod}_{F}^{\text {good }}\left(\mathscr{W}\left[\hbar^{1 / m}\right]\right)$ is equivalent to $\operatorname{Mod}_{\text {coh }}\left(\mathscr{D}\left(\mathbb{C}^{n}\right)\right)$ (see Theorem 2.10 below).

2.4. Equivariance. We shall discuss $G$-equivariance of $\mathscr{W}$ by adapting [18, 17] where the $\mathscr{D}$-module version is studied. 
2.4.1. Let $G$ be a complex Lie group acting on a symplectic manifold $X$. Given $g \in G$, let $T_{g}$ be the corresponding symplectic automorphism of $X$. Let $\mathfrak{g}$ be the Lie algebra of $G$ and assume that a moment map $\mu_{X}: X \rightarrow \mathfrak{g}^{*}$ is given.

A W-algebra with $G$-action is a W-algebra with an action of $G$ : we have k-algebra isomorphisms $\rho_{g}: \mathscr{W} \stackrel{\sim}{\longrightarrow} T_{g}^{-1} \mathscr{W}$ for $g \in G$ such that for any $a \in \mathscr{W}, \rho_{g}(a)$ depends holomorphically on $g \in G$. Moreover we assume that there is a quantized moment map $\mu_{\mathscr{W}}: \mathfrak{g} \rightarrow \mathscr{W}(1)$ such that

$$
\begin{aligned}
& {\left[\mu_{\mathscr{W}}(A), a\right]=\left.\frac{d}{d t} \rho_{\exp (t A)}(a)\right|_{t=0},} \\
& \sigma_{0}\left(\hbar \mu_{\mathscr{W}}(A)\right)=A \circ \mu_{X}, \\
& \mu_{\mathscr{W}}(\operatorname{Ad}(g) A)=\rho_{g}\left(\mu_{\mathscr{W}}(A)\right)
\end{aligned}
$$

for any $A \in \mathfrak{g}$ and $a \in \mathscr{W}$.

Note that $\mu_{\mathscr{W}}$ is a Lie algebra homomorphism.

2.4.2. A quasi-G-equivariant $\mathscr{W}$-module is a $\mathscr{W}$-module $\mathscr{M}$ with an action of $G$ :

$$
\rho_{g}: \mathscr{M} \stackrel{\sim}{\longrightarrow} T_{g}^{-1} \mathscr{M}
$$

depending holomorphically on $g \in G$ and such that $\rho_{g}(a u)=\rho_{g}(a) \rho_{g}(u)$ for $a \in \mathscr{W}$ and $u \in \mathscr{M}$. Then, we have a Lie algebra homomorphism $\alpha: \mathfrak{g} \rightarrow \operatorname{End}_{\mathbf{k}}(\mathscr{M})$ given by $\alpha(A)(u)=\left.\frac{d}{d t} \rho_{\exp (t A)} u\right|_{t=0}$ for $A \in \mathfrak{g}$ and $u \in \mathscr{M}$. It satisfies

$$
\alpha(A)(a u)=\left[\mu_{\mathscr{W}}(A), a\right] u+a \cdot \alpha(A)(u) .
$$

It follows that we have a Lie algebra homomorphism

$$
\gamma_{\mathscr{M}}: \mathfrak{g} \rightarrow \operatorname{End}_{\mathscr{W}}(\mathscr{M}), A \mapsto \alpha(A)-\mu_{\mathscr{W}}(A) .
$$

The $\mathscr{W}$-module $\mathscr{W}$ is regarded as a quasi- $G$-equivariant $\mathscr{W}$-module. We have $\alpha(A)=$ $\operatorname{ad}\left(\mu_{\mathscr{W}}(A)\right)$ and $\gamma_{\mathscr{W}}(A)(a)=-a \mu_{\mathscr{W}}(A)(a \in \mathscr{W}, A \in \mathfrak{g})$. Given a $G$-module $V$ and a quasi- $G$-equivariant $\mathscr{W}$-module $\mathscr{M}$, the tensor product $\mathscr{M} \otimes V$ has a natural structure of a quasi- $G$-equivariant $\mathscr{W}$-module. The corresponding $\gamma$ is given by

$$
\gamma_{\mathscr{M} \otimes V}(A)(u \otimes v)=\gamma_{\mathscr{M}}(A) u \otimes v+u \otimes A v \quad \text { for } u \in \mathscr{M}, v \in V \text { and } A \in \mathfrak{g} .
$$

Let $\lambda \in\left(\mathfrak{g}^{*}\right)^{G}$. If $\gamma_{\mathscr{M}}$ coincides with the composition $\mathfrak{g} \stackrel{\lambda}{\rightarrow} \mathbb{C} \stackrel{z \mapsto z \cdot \operatorname{Id} \mathscr{M}}{\rightarrow} \operatorname{End}_{\mathscr{W}}(\mathscr{M})$, we say that $\mathscr{M}$ is a twisted $G$-equivariant $\mathscr{W}$-module with twist $\lambda$. For such a coherent module $\mathscr{M}$, we have $\operatorname{Supp}(\mathscr{M}) \subset \mu_{X}^{-1}(0)$.

We denote by $\operatorname{Mod}(\mathscr{W}, G)$ the category of quasi- $G$-equivariant $\mathscr{W}$-modules, and by $\operatorname{Mod}_{\lambda}^{G}(\mathscr{W})$ its full subcategory of twisted $G$-equivariant $\mathscr{W}$-modules with twist $\lambda$. We denote by $\operatorname{Mod}_{\lambda}^{G \text {, good }}(\mathscr{W})$ the category of good twisted $G$-equivariant $\mathscr{W}$-modules with twist $\lambda$.

The embedding $\operatorname{Mod}_{\lambda}^{G}(\mathscr{W}) \rightarrow \operatorname{Mod}(\mathscr{W}, G)$ has a left adjoint

$$
\begin{gathered}
\Phi_{\lambda}: \operatorname{Mod}(\mathscr{W}, G) \rightarrow \operatorname{Mod}_{\lambda}^{G}(\mathscr{W}) \\
\Phi_{\lambda}(\mathscr{M})=\mathscr{M} /\left(\sum_{A \in \mathfrak{g}}\left(\gamma_{\mathscr{M}}(A)-\lambda(A)\right) \mathscr{M}\right) .
\end{gathered}
$$

Let $V$ be a one-dimensional $G$-module and $\chi \in\left(\mathfrak{g}^{*}\right)^{G}$ its infinitesimal character. Then, we have an equivalence

$$
\operatorname{Mod}_{\lambda}^{G}(\mathscr{W}) \stackrel{\sim}{\longrightarrow} \operatorname{Mod}_{\lambda+\chi}^{G}(\mathscr{W}), \mathscr{M} \mapsto \mathscr{M} \otimes V
$$


Let $\mathscr{W}$ be a $\mathrm{W}$-algebra with an $\mathrm{F}$-action with exponent $m$. A $G$-action on $(\mathscr{W}, \mathcal{F})$ is a $G$-action on $\mathscr{W}$ such that $T_{t}$ and $T_{g}$ commute, $\mathcal{F}_{t}$ and $\rho(g)$ commute and $\mu_{\mathscr{W}}(A)$ is $\mathcal{F}_{t}$-invariant, for $t \in \mathbb{C}^{\times}, g \in G$ and $A \in \mathfrak{g}$.

We define similarly the notion of twisted $G$-equivariant $\left(\mathscr{W}\left[\hbar^{1 / m}\right], \mathcal{F}\right)$-modules. We denote by $\operatorname{Mod}_{F, \lambda}^{G \text {, good }}\left(\mathscr{W}\left[\hbar^{1 / m}\right]\right)$ the category of good twisted $G$-equivariant $\left(\mathscr{W}\left[\hbar^{1 / m}\right], \mathcal{F}\right)$ modules with twist $\lambda \in\left(\mathfrak{g}^{*}\right)^{G}$.

2.5. Symplectic reduction. Let $X$ be a symplectic manifold with a symplectic action of $G$ and a moment map $\mu_{X}: X \rightarrow \mathfrak{g}^{*}$. Assume that $G$ acts properly and freely on $X$ (i.e., the map $G \times X \rightarrow X \times X$ defined by $(g, x) \mapsto(g x, x)$ is a closed embedding $)$. Then, $\mu_{X}^{-1}(0)$ is an involutive submanifold. Let $Z=\mu_{X}^{-1}(0) / G$, and let $p: \mu_{X}^{-1}(0) \rightarrow Z$ be the projection. Then $Z$ carries a natural symplectic structure such that $p$ preserves the symplectic form (i.e., denoting by $\omega_{Z}$ the symplectic form of $Z$, we have $\left.p^{*} \omega_{Z}=\left.\omega_{X}\right|_{\mu_{X}^{-1}(0)}\right)$. The local form of $X$ is given by the following Lemma [10, $\S 41$.

Lemma 2.7. Locally on $Z$, the manifold $X$ is isomorphic to $T^{*} G \times Z$. More precisely, for any point $x \in \mu_{X}^{-1}(0)$, there exist a $G$-invariant open neighbourhood $U$ of $x$ in $X$ and $a$ $G$-equivariant open symplectic embedding $U \rightarrow T^{*} G \times T^{*} \mathbb{C}^{n}$ compatible with the moment maps.

Let $\mathscr{W}$ be a $\mathrm{W}$-algebra on $X$ with a $G$-action. Let $\lambda \in\left(\mathfrak{g}^{*}\right)^{G}$. Set

$$
\mathscr{L}_{\lambda}:=\Phi_{\lambda}(\mathscr{W})=\mathscr{W} / \sum_{A \in \mathfrak{g}} \mathscr{W}\left(\mu_{\mathscr{W}}(A)+\lambda(A)\right) .
$$

Then, $\mathscr{L}_{\lambda}$ is a coherent twisted $G$-equivariant $\mathscr{W}$-module with twist $\lambda$. The support of $\mathscr{L}_{\lambda}$ coincides with $\mu_{X}^{-1}(0)$. Let $\mathscr{L}_{\lambda}(0)$ be the $\mathscr{W}(0)$-lattice $\mathscr{W}(0) / \sum_{A \in \mathfrak{g}} \mathscr{W}(-1)\left(\mu_{\mathscr{W}}(A)+\lambda(A)\right)$ of $\mathscr{L}_{\lambda}$.

Let $\mathscr{W}_{Z}=\left(\left(p_{*} \mathscr{E} n d_{\mathscr{W}}\left(\mathscr{L}_{\lambda}\right)\right)^{G}\right)^{\text {opp }}$, a sheaf of k-algebras on $Z$.

Proposition 2.8. (i) $\mathscr{W}_{Z}$ is a W-algebra on $Z$, and $\mathscr{W}_{Z}(0) \simeq\left(\left(p_{*} \mathscr{E} n d_{\mathscr{W}(0)}\left(\mathscr{L}_{\lambda}(0)\right)\right)^{G}\right)^{\text {opp }}$.

(ii) We have quasi-inverse equivalences of categories

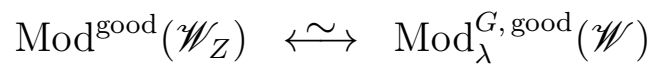

$$
\begin{aligned}
& \mathscr{N} \mapsto \mathscr{L}_{\lambda} \otimes_{p^{-1} \mathscr{W}_{Z}} p^{-1} \mathscr{N} \\
& \left(p_{*} \mathscr{H}_{0} m_{\mathscr{W}}\left(\mathscr{L}_{\lambda}, \mathscr{M}\right)\right)^{G} \longleftrightarrow \quad \mathscr{M} .
\end{aligned}
$$

(iii) Let $V$ be a one-dimensional representation with infinitesimal character $\chi$. Then $\mathscr{N}_{\lambda, \chi}(0):=\left(p_{*} \mathscr{H}_{o m_{\mathscr{W}}(0)}\left(\mathscr{L}_{\lambda}(0), \mathscr{L}_{\lambda-\chi}(0) \otimes V\right)\right)^{G}$ is a $\mathscr{W}_{Z}(0)$-lattice of a coherent $\mathscr{W}_{Z}$ module $\mathscr{N}_{\lambda, \chi}:=\left(p_{*} \mathscr{H}_{0 m}\left(\mathscr{L}_{\lambda}, \mathscr{L}_{\lambda-\chi} \otimes V\right)\right)^{G}$ and $\mathscr{N}_{\lambda, \chi}(0) / \hbar \mathscr{N}_{\lambda, \chi}(0)$ is isomorphic to $\left(p_{*}\left(\mathscr{O}_{\mu_{X}^{-1}(0)} \otimes V\right)\right)^{G}$, the line bundle on $Z$ associated with $V$.

(iv) Assume that $\mathscr{W}$ has an F-action with exponent $m$ compatible with the $G$-action. Then $\mathscr{W}_{Z}$ has a natural F-action with exponent $m$ and we have an equivalence of categories:

$$
\operatorname{Mod}_{F}^{\text {good }}\left(\mathscr{W}_{Z}\left[\hbar^{1 / m}\right]\right) \simeq \operatorname{Mod}_{F, \lambda}^{G, \operatorname{good}}\left(\mathscr{W}\left[\hbar^{1 / m}\right]\right)
$$

Note that $\mathscr{H}_{\mathscr{H}} m_{\mathscr{W}}\left(\mathscr{L}_{\lambda}, \mathscr{M}\right) \simeq p^{-1}\left(\left(p_{*} \mathscr{H}_{o} m_{\mathscr{W}}\left(\mathscr{L}_{\lambda}, \mathscr{M}\right)\right)^{G}\right)$. Hence, if $G$ is connected, we have $p_{*} \mathscr{H}_{0} m_{\mathscr{W}}\left(\mathscr{L}_{\lambda}, \mathscr{M}\right) \simeq\left(p_{*} \mathscr{H}_{0} m_{\mathscr{W}}\left(\mathscr{L}_{\lambda}, \mathscr{M}\right)\right)^{G}$.

\section{6. $\mathscr{W}$-affinity.}


2.6.1. Let $X$ be a symplectic manifold. Let $S$ be a variety, let $f: X \rightarrow S$ be a projective morphism, and let $L$ be a relatively ample line bundle on $X$. Let $\mathscr{W}$ be a $W$-algebra on $X$. The following theorem is an analogue of the result of Beilinson-Bernstein [1] on $\mathscr{D}$-modules on flag manifolds. We follow the formulation of [17].

Theorem 2.9. For $n>0$, let $\mathscr{L}_{n}(0)$ be a locally free $\mathscr{W}(0)$-module of rank 1 such that $\mathscr{L}_{n}(0) / \hbar \mathscr{L}_{n}(0)=L^{\otimes(-n)}$. Set $\mathscr{L}_{n}=\mathscr{W} \otimes \mathscr{W}(0) \mathscr{L}_{n}(0)$.

Consider the conditions:

for $n \gg 0$, there exists a vector space $V_{n}$ and a split epimorphism $\mathscr{L}_{n} \otimes V_{n} \rightarrow \mathscr{W}$, i.e., $\mathscr{W}$ is a direct summand of the direct sum of finitely many copies of $\mathscr{L}_{n}$;

for $n \gg 0$, there exists a vector space $V_{n}$ and an epimorphism $\mathscr{W} \otimes V_{n} \rightarrow$ $\mathscr{L}_{n}$.

(i) Assume (2.5). Then, for every good $\mathscr{W}$-module $\mathscr{M}$, we have $R^{i} f_{*}(\mathscr{M})=0$ for $i \neq 0$.

(ii) Assume (2.6). Then, every good $\mathscr{W}$-module is generated by its global sections (locally on $S$ ).

The proof will be given in the next two subsections.

Assume that $\mathscr{W}$ has an F-action with exponent $m$ and that $S$ has a $\mathbb{G}_{\mathrm{m}}$-action such that $f$ is $\mathbb{G}_{\mathrm{m}}$-equivariant. Assume moreover that there exists o $\in S$ such that every point of $S$ shrinks to o (i.e., $\lim _{t \rightarrow 0} t x=$ o for any $x \in S$ ).

Let $\widetilde{\mathscr{W}}=\mathscr{W}\left[\hbar^{1 / m}\right]$ and $A=\operatorname{End}_{\operatorname{Mod}_{F}(\widetilde{\mathscr{W}})}(\widetilde{\mathscr{W}})^{\text {opp }}$.

Theorem 2.10. Assume Conditions (2.5) and (2.6) hold. Then, A is a left noetherian ring and we have quasi-inverse equivalences of categories between $\operatorname{Mod}_{F}^{\text {good }}(\widetilde{\mathscr{W}})$ and $\operatorname{Mod}_{\text {coh }}(A)$

$$
\begin{aligned}
\operatorname{Mod}_{F}^{\text {good }}(\widetilde{\mathscr{W}}) & \stackrel{\leftrightarrow}{\sim} \operatorname{Mod}_{\mathrm{coh}}(A) \\
\mathscr{M} & \mapsto \operatorname{Hom}_{\operatorname{Mod}_{F}^{\text {good }}(\widetilde{\mathscr{W}})}(\widetilde{\mathscr{W}}, \mathscr{M}) \\
\widetilde{\mathscr{W}} \otimes_{A} M & \hookleftarrow M .
\end{aligned}
$$

The proof will be given in $\S 2.6 .4$.

2.6.2. Vanishing theorem. Let $\mathscr{W}$ be a $\mathrm{W}$-algebra on a symplectic manifold $X$. Let $\mathscr{M}$ be a coherent $\mathscr{W}$-module. Recall that $\mathscr{M}(0)$ is a $\mathscr{W}(0)$-lattice of $\mathscr{M}$ if $\mathscr{M}(0)$ is a coherent $\mathscr{W}(0)$-submodule of $\mathscr{M}$ such that $\mathscr{W} \otimes \mathscr{W}(0) \mathscr{M}(0) \stackrel{\sim}{\longrightarrow}$.

We start with the following lemma.

Lemma 2.11. For any coherent $\mathscr{W}(0)$-module $\mathscr{N}$, the canonical map is an isomorphism

$$
\mathscr{N} \stackrel{\sim}{\longrightarrow} \lim _{m} \mathscr{N} / \hbar^{m} \mathscr{N} \text {. }
$$

Proof. Let us first show that $\mathscr{N} \rightarrow \underset{m}{\lim _{m}} \mathscr{N} / \hbar^{m} \mathscr{N}$ is a monomorphism. For any $x \in X$, we have morphisms of $\mathscr{W}(0)_{x}$-modules:

$$
\mathscr{N}_{x} \rightarrow\left(\varliminf_{m}^{\lim } \mathscr{N} / \hbar^{m} \mathscr{N}\right)_{x} \rightarrow \underset{m}{\lim }\left(\mathscr{N}_{x} / \hbar^{m} \mathscr{N}_{x}\right) \text {. }
$$


Since the composition is injective (Artin-Rees argument, see e.g. [25]), the map $\mathscr{N}_{x} \rightarrow$ $\left(\lim _{m} \mathscr{N} / \hbar^{m} \mathscr{N}\right)_{x}$ is injective.

Let us show now that $\mathscr{N} \rightarrow \underset{m}{\lim } \mathscr{N} / \hbar^{m} \mathscr{N}$ is surjective. The question being local, we can take an exact sequence of coherent $\mathscr{W}(0)$-modules

$$
0 \rightarrow \mathscr{M} \rightarrow \mathscr{L} \rightarrow \mathscr{N} \rightarrow 0
$$

where $\mathscr{L}$ is a free $\mathscr{W}(0)$-module of finite rank. For any Stein open subset $U$ and $m>0$, we have

$$
H^{1}\left(U, \mathscr{M} /\left(\hbar^{m} \mathscr{L} \cap \mathscr{M}\right)\right)=0
$$

and

$$
\Gamma\left(U, \mathscr{M} /\left(\hbar^{m} \mathscr{L} \cap \mathscr{M}\right)\right) \rightarrow \Gamma\left(U, \mathscr{M} /\left(\hbar^{m-1} \mathscr{L} \cap \mathscr{M}\right)\right) \text { is surjective. }
$$

Indeed, in the exact sequence

$$
\begin{aligned}
\Gamma\left(U ; \mathscr{M} /\left(\hbar^{m} \mathscr{L} \cap \mathscr{M}\right)\right) \rightarrow \Gamma\left(U ; \mathscr{M} /\left(\hbar^{m-1} \mathscr{L} \cap \mathscr{M}\right)\right) \\
\rightarrow H^{1}\left(U ;\left(\hbar^{m-1} \mathscr{L} \cap \mathscr{M}\right) /\left(\hbar^{m} \mathscr{L} \cap \mathscr{M}\right)\right) \\
\quad \rightarrow H^{1}\left(U ; \mathscr{M} /\left(\hbar^{m} \mathscr{L} \cap \mathscr{M}\right)\right) \rightarrow H^{1}\left(U ; \mathscr{M} /\left(\hbar^{m-1} \mathscr{L} \cap \mathscr{M}\right)\right),
\end{aligned}
$$

$H^{1}\left(U ;\left(\hbar^{m-1} \mathscr{L} \cap \mathscr{M}\right) /\left(\hbar^{m} \mathscr{L} \cap \mathscr{M}\right)\right)$ vanishes because $\left(\hbar^{m-1} \mathscr{L} \cap \mathscr{M}\right) /\left(\hbar^{m} \mathscr{L} \cap \mathscr{M}\right)$ is a coherent $\mathscr{O}_{X}$-module.

It follows that the following sequence is exact

$$
0 \rightarrow \Gamma\left(U, \mathscr{M} /\left(\hbar^{m} \mathscr{L} \cap \mathscr{M}\right)\right) \rightarrow \Gamma\left(U, \mathscr{L} / \hbar^{m} \mathscr{L}\right) \rightarrow \Gamma\left(U, \mathscr{N} / \hbar^{m} \mathscr{N}\right) \rightarrow 0 .
$$

Since $\left\{\Gamma\left(U, \mathscr{M} /\left(\hbar^{m} \mathscr{L} \cap \mathscr{M}\right)\right)\right\}_{m}$ satisfies the ML condition, the bottom row of the following commutative diagram is exact

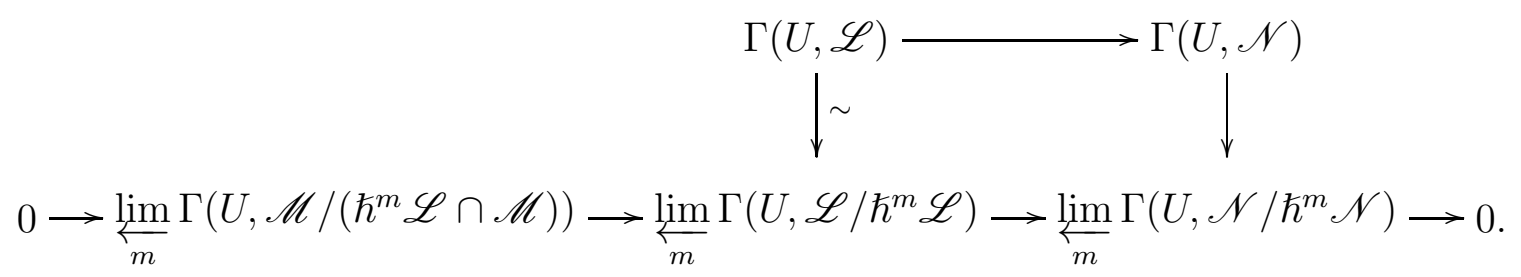

It follows that $\Gamma(U, \mathscr{N}) \rightarrow \underset{\lim _{m}}{\lim } \Gamma\left(U, \mathscr{N} / \hbar^{m} \mathscr{N}\right) \simeq \Gamma\left(U, \underset{m}{\lim _{m}} \mathscr{N} / \hbar^{m} \mathscr{N}\right)$ is surjective.

Lemma 2.12. Let $\mathscr{M}$ be a coherent $\mathscr{W}$-module and let $\mathscr{M}(0)$ be a $\mathscr{W}(0)$-lattice of $\mathscr{M}$. Set $\mathscr{M}(m)=\hbar^{-m} \mathscr{M}(0)$ and $\overline{\mathscr{M}}=\mathscr{M}(0) / \mathscr{M}(-1)$. Assume that

$$
H^{i}(X, \overline{\mathscr{M}})=0 \text { for } i \neq 0 \text {. }
$$

Then,

(i) the canonical morphism

$$
\Gamma(X, \mathscr{M}(0)) / \Gamma(X, \mathscr{M}(-m)) \longrightarrow \Gamma(X, \mathscr{M}(0) / \mathscr{M}(-m))
$$

is an isomorphism for any $m \geqslant 0$,

(ii) $H^{i}(X, \mathscr{M}(0))=0$ for any $i \neq 0$. 
Proof. Given $m \geqslant 0$, the exact sequence

$$
0 \rightarrow \overline{\mathscr{M}} \stackrel{\hbar^{m}}{\longrightarrow} \mathscr{M}(0) / \mathscr{M}(-m-1) \rightarrow \mathscr{M}(0) / \mathscr{M}(-m) \rightarrow 0
$$

induces exact sequences

$$
\Gamma(X, \mathscr{M}(0) / \mathscr{M}(-m-1)) \rightarrow \Gamma(X, \mathscr{M}(0) / \mathscr{M}(-m)) \rightarrow H^{1}(X, \overline{\mathscr{M}})
$$

and

$$
H^{i}(X, \overline{\mathscr{M}}) \rightarrow H^{i}(X, \mathscr{M}(0) / \mathscr{M}(-m-1)) \rightarrow H^{i}(X, \mathscr{M}(0) / \mathscr{M}(-m)) .
$$

It follows that $\Gamma(X, \mathscr{M}(0) / \mathscr{M}(-m-1)) \rightarrow \Gamma(X, \mathscr{M}(0) / \mathscr{M}(-m))$ is surjective for any $m \geqslant$ 0 and $H^{i}(X, \mathscr{M}(0) / \mathscr{M}(-m))=0$ for any $i>0$. Since $\Gamma(X, \mathscr{M}(0))={\underset{\lim }{m}} \Gamma(X, \mathscr{M}(0) / \mathscr{M}(-m))$

by Lemma 2.11, we obtain (i).

For $i>0$, we have

$$
H^{i}(X, \mathscr{M}(0))={\underset{\lim }{m}} H^{i}(X, \mathscr{M}(0) / \mathscr{M}(-m))=0
$$

because $\left\{H^{i-1}(X, \mathscr{M}(0) / \mathscr{M}(-m))\right\}_{m}$ satisfies the ML condition.

2.6.3. Proof of Theorem 2.9. Let us prove (i). The question being local on $S$, we may assume that there exists a $\mathscr{W}(0)$-lattice $\mathscr{M}(0)$ of $\mathscr{M}$. Set $\overline{\mathscr{M}}=\mathscr{M}(0) / \hbar \mathscr{M}(0)$. Then, for $m \gg 0$, we have $R^{i} f_{*}\left(L^{\otimes m} \otimes_{\mathscr{O}_{X}} \overline{\mathscr{M}}\right)=0$ for $i \neq 0$. It follows that

$$
H^{i}\left(f^{-1} U, L^{\otimes m} \otimes_{\mathscr{O}_{X}} \overline{\mathscr{M}}\right)=0
$$

for any $i \neq 0$ and any Stein open subset $U$ of $S$. From now on, we assume that $m$ is large enough so that the vanishing above holds.

Let $\mathscr{A}_{m}=\mathscr{E} n d_{\mathscr{W}}\left(\mathscr{L}_{m}\right)^{\text {opp}}$, a W-algebra on $X$. We have $\mathscr{A}_{m}(0)=\mathscr{E} n d_{\mathscr{W}(0)}\left(\mathscr{L}_{m}(0)\right)^{\text {opp }}$. Let $\mathscr{L}_{m}(0)^{*}=\mathscr{H}_{o m_{\mathscr{W}}(0)}\left(\mathscr{L}_{m}(0), \mathscr{W}(0)\right)$, an $\left(\mathscr{A}_{m}(0), \mathscr{W}(0)\right)$-bimodule, and let $\mathscr{L}_{m}^{*}=$ $\mathscr{H}_{o} m_{\mathscr{W}}\left(\mathscr{L}_{m}, \mathscr{W}\right)$, an $\left(\mathscr{A}_{m}, \mathscr{W}\right)$-bimodule. We have

$$
\mathscr{L}_{m}^{*} \simeq \mathscr{A}_{m} \otimes_{\mathscr{A}_{m}(0)} \mathscr{L}_{m}(0)^{*} \simeq \mathscr{L}_{m}(0)^{*} \otimes_{\mathscr{W}(0)} \mathscr{W} .
$$

Note that the bimodules $\mathscr{L}_{m}$ and $\mathscr{L}_{m}^{*}$ give inverse Morita equivalences between $\mathscr{A}_{m}$ and W.

Let $\mathscr{M}_{m}(0)=\mathscr{L}_{m}^{*}(0) \otimes_{\mathscr{W}(0)} \mathscr{M}(0)$, an $\mathscr{A}_{m}(0)$-lattice in the $\mathscr{A}_{m}$-module $\mathscr{M}_{m}=\mathscr{L}_{m}^{*} \otimes \mathscr{W} \mathscr{M}$. We have $\mathscr{M}_{m}(0) / \hbar \mathscr{M}_{m}(0) \simeq L^{\otimes m} \otimes_{\mathscr{O}_{X}} \overline{\mathscr{M}}$, hence $H^{i}\left(f^{-1} U, \mathscr{M}_{m}(0) / \hbar \mathscr{M}_{m}(0)\right)=0$ for $i \neq 0$. Lemma 2.12 (ii) implies that $H^{i}\left(f^{-1} U, \mathscr{M}_{m}(0)\right)=0$ for $i \neq 0$. Taking the inductive limit with respect to Stein open neighbourhoods $U$ of $s \in S$, we obtain $H^{i}\left(f^{-1}(s), \mathscr{M}_{m}(0)\right)=0$, hence

$$
H^{i}\left(f^{-1}(s), \mathscr{M}_{m}\right) \simeq \mathbf{k} \otimes_{\mathbf{k}(0)} H^{i}\left(f^{-1}(s), \mathscr{M}_{m}(0)\right)=0 .
$$

By Condition (2.5), $\mathscr{W}$ is a direct summand of a direct sum of finitely many copies of the left $\mathscr{W}$-module $\mathscr{L}_{m}$. So, $\mathscr{W}$ is a direct summand of a direct sum of finitely many copies of the right $\mathscr{W}$-module $\mathscr{L}_{m}^{*}$ and $\mathscr{M}$ is a direct summand of a direct sum of finitely many copies of $\mathscr{M}_{m}$ (as a sheaf). Then, (2.8) implies that $H^{i}\left(f^{-1}(s), \mathscr{M}\right)=0$. This completes the proof of (i).

We now prove (ii). We shall keep the same notations as in the proof of (i). Since $L$ is relatively ample, given $s \in S$, there exists a surjective map $\left(\left.\mathscr{O}_{X}\right|_{f^{-1}(s)}\right)^{\oplus N} \rightarrow$ $\left.\left(L^{\otimes m} \otimes \overline{\mathscr{M}}\right)\right|_{f^{-1}(s)}$ for some $N$. On the other hand, Lemma 2.12 (i) implies that 
$\Gamma\left(f^{-1}(s), \mathscr{M}_{m}(0)\right) \rightarrow \Gamma\left(f^{-1}(s), \mathscr{M}_{m}(0) / \hbar \mathscr{M}_{m}(0)\right)$ is surjective. Hence we have a morphism $\phi_{m}:\left.\left.\mathscr{A}_{m}(0)^{\oplus N}\right|_{f^{-1}(s)} \rightarrow \mathscr{M}_{m}(0)\right|_{f^{-1}(s)}$ such that the composition $\left.\mathscr{A}_{m}(0)^{\oplus N}\right|_{f^{-1}(s)} \rightarrow$ $\left.\left(\mathscr{M}_{m}(0) / \hbar \mathscr{M}_{m}(0)\right)\right|_{f^{-1}(s)}$ is an epimorphism. It follows that $\phi_{m}$ is an epimorphism. Thus, there exists an epimorphism $\left.\left.\mathscr{A}_{m}^{\oplus N}\right|_{f^{-1}(s)} \rightarrow \mathscr{M}_{m}\right|_{f^{-1}(s)}$. By applying the exact functor $\mathscr{L}_{m} \otimes_{\mathscr{A}_{m}} \cdot: \operatorname{Mod}\left(\mathscr{A}_{m}\right) \rightarrow \operatorname{Mod}(\mathscr{W})$, we obtain an epimorphism $\left.\mathscr{L}_{m}^{\oplus N}\right|_{f^{-1}(s)} \rightarrow \mathscr{M}_{f^{-1}(s)}$. The assertion follows now from Condition (2.6).

2.6.4. Proof of Theorem 2.10. By Theorem 2.9, $\operatorname{Mod}_{F}^{\text {good }}(\widetilde{\mathscr{W}}) \ni \mathscr{M} \mapsto f_{*}(\mathscr{M}) \in \operatorname{Mod}\left(f_{*}(\widetilde{\mathscr{W}})\right)$ is an exact functor.

By the assumption, o has a neighbourhood system consisting of relatively compact Stein open neighbourhoods $U$ such that $U$ is stable by $T_{t}(0<|t| \leqslant 1)$. For such an $U$, we have $S=\bigcup_{t \in \mathbb{C}^{*}} T_{t} U$. For any $\mathscr{M} \in \operatorname{Mod}_{F}^{\text {good }}(\widetilde{\mathscr{W}})$, we have

$$
\operatorname{Hom}_{\operatorname{Mod}_{F}(\widetilde{\mathscr{W}})}(\widetilde{\mathscr{W}}, \mathscr{M})=\left\{s \in \mathscr{M}\left(f^{-1} U\right) ; s \text { is F-invariant }\right\} .
$$

Here $s \in \mathscr{M}\left(f^{-1} U\right)$ is F-invariant if $\mathcal{F}_{t}(s)=s$ for any $t \in \mathbb{C}^{\times}$with $|t|=1$.

For $s \in \mathscr{M}\left(f^{-1} U\right)$, let

$$
p_{n}(s)=\frac{1}{2 \pi \sqrt{-1}} \int_{|t|=1} t^{-n} \mathcal{F}_{t}(s) \frac{d t}{t} .
$$

We have $s=\sum_{n} p_{n}(s)$ and $\hbar^{-n / m} p_{n}(s)=p_{0}\left(\hbar^{-n / m} s\right)$ is F-invariant.

Lemma 2.13. $\operatorname{Hom}_{\operatorname{Mod}_{F}^{\operatorname{good}}(\widetilde{\mathscr{W}})}(\widetilde{\mathscr{W}}, \bullet)$ is an exact functor.

Proof. Let $\varphi: \mathscr{M} \rightarrow \mathscr{M}^{\prime} \rightarrow 0$ be an epimorphism in $\operatorname{Mod}_{F}^{\text {good }}(\widetilde{\mathscr{W}})$ and let $s^{\prime} \in \mathscr{M}^{\prime}\left(f^{-1} U\right)$ such that $\mathcal{F}_{t}\left(s^{\prime}\right)=s^{\prime}$ for any $t$ with $|t|=1$. By Theorem 2.9, there exists $s \in \mathscr{M}\left(f^{-1} U\right)$ such that $\varphi(s)=s^{\prime}$. We have $\varphi\left(p_{0}(s)\right)=s^{\prime}$ and $p_{0}(s)$ is F-invariant.

Lemma 2.14. Any $\mathscr{M} \in \operatorname{Mod}_{F}^{\text {good }}(\widetilde{\mathscr{W}})$ is generated by F-invariant global sections.

Proof. By Theorem 2.9, $\mathscr{M}$ is generated by global sections $s_{i} \in \mathscr{M}\left(f^{-1} U\right)$. Then, $\mathscr{M}$ is generated by the $\hbar^{-n / m} p_{n}\left(s_{i}\right)$ 's. Indeed, let $\mathscr{N}$ be the submodule of $\mathscr{M}$ generated by the $p_{n}\left(s_{i}\right)^{\prime}$ s. This is a coherent submodule of $\mathscr{M}$. Let $\psi: \mathscr{M} \rightarrow \mathscr{M} / \mathscr{N}$ be the quotient morphism. Then $p_{n} \psi\left(s_{i}\right)=\psi\left(p_{n}\left(s_{i}\right)\right)=0$ for any $n$, and hence $\psi\left(s_{i}\right)=0$. It follows that $\mathscr{N}=\mathscr{M}$.

We deduce that $\operatorname{Hom}_{\operatorname{Mod}_{F}^{\text {good }}(\widetilde{\mathscr{W}})}(\widetilde{\mathscr{W}}, \mathscr{M})$ is an $A$-module of finite presentation for any $\mathscr{M} \in \operatorname{Mod}_{F}^{\operatorname{good}}(\widetilde{\mathscr{W}})$.

Lemma 2.15. A is left noetherian.

Proof. Let $I$ be a left ideal of $A$. Let $\mathscr{I} \subset \widetilde{\mathscr{W}}$ be the image of $\widetilde{\mathscr{W}} \otimes_{A} I \rightarrow \widetilde{\mathscr{W}}$. Note that $\mathscr{I}$ belongs to $\operatorname{Mod}_{F}^{\text {good }}(\widetilde{\mathscr{W}})$. Since $\widetilde{\mathscr{W}}$ is coherent, there exist finitely many $a_{i} \in I$ such that $\mathscr{I}=\sum \widetilde{\mathscr{W}} a_{i}$. We have $\operatorname{Hom}_{\operatorname{Mod}_{F}^{\text {good }}(\widetilde{\mathscr{W}})}(\widetilde{\mathscr{W}}, \mathscr{I})=\sum_{i} A a_{i} \subset I$ by Lemma 2.13. Since we have injective maps $I \rightarrow \operatorname{Hom}_{\operatorname{Mod}_{F}^{\operatorname{good}}(\widetilde{\mathscr{W}})}(\widetilde{\mathscr{W}}, \mathscr{I}) \hookrightarrow \operatorname{Hom}_{\operatorname{Mod}_{F}^{\operatorname{good}}(\widetilde{\mathscr{W}})}(\widetilde{\mathscr{W}}, \widetilde{\mathscr{W}})=A$, we obtain $I=\sum_{i} A a_{i}$.

Since $\operatorname{good}(\widetilde{\mathscr{W}}, F)$-modules are generated by F-invariant sections, $\operatorname{Hom}_{\operatorname{Mod}_{F}(\widetilde{\mathscr{W}})}(\widetilde{\mathscr{W}}, \bullet)$ $\operatorname{sends} \operatorname{Mod}_{F}^{\text {good }}(\widetilde{\mathscr{W}})$ to $\operatorname{Mod}_{\text {coh }}(A)$. 
Given $M \in \operatorname{Mod}_{\text {coh }}(A)$, the canonical morphism

$$
M \rightarrow \operatorname{Hom}_{\operatorname{Mod}_{F}^{\operatorname{good}}(\widetilde{\mathscr{W}})}\left(\widetilde{\mathscr{W}}, \widetilde{\mathscr{W}} \otimes_{A} M\right)
$$

is an isomorphism because both sides are right exact functors of $M$ and the morphism is an isomorphism for $M=A$.

Given $\mathscr{M} \in \operatorname{Mod}_{F}^{\text {good }}(\widetilde{\mathscr{W}})$, the canonical map $\widetilde{\mathscr{W}} \otimes_{A} \operatorname{Hom}_{\operatorname{Mod}_{F}^{\text {good }}(\widetilde{\mathscr{W}})}(\widetilde{\mathscr{W}}, \mathscr{M}) \rightarrow \mathscr{M}$ is an isomorphism, because both sides are right exact functors of $\mathscr{M}$ and $\mathscr{M}$ has a resolution $\widetilde{\mathscr{W}} \oplus m_{1} \rightarrow \widetilde{\mathscr{W}} \oplus m_{0} \rightarrow \mathscr{M} \rightarrow 0$ in $\operatorname{Mod}_{F}^{\text {good }}(\widetilde{\mathscr{W}})$ by Lemma 2.14

This completes the proof of Theorem 2.10.

\section{Rational Cherednik algebras and $\mathscr{D}$-modules}

\subsection{Definitions, notations and recollections.}

3.1.1. Let $V=\mathbb{C}^{n}$, let $G=\mathrm{GL}(V)=\mathrm{GL}_{n}(\mathbb{C})$ and let $\mathfrak{g}=\mathfrak{g l}(V)=\mathfrak{g l}_{n}(\mathbb{C})$. We denote by $e_{r s} \in \mathfrak{g}$ the elementary matrix with 0 coefficients everywhere except in row $r$ and column $s$ where the coefficient is 1 . We denote by $A_{r s} \in \mathbb{C}[\mathfrak{g}]$ the corresponding coordinate function.

We denote by $\mathfrak{t}=\mathbb{C}^{n}$ the Cartan subalgebra of diagonal matrices of $\mathfrak{g}$ and by $W=S_{n}$ the Weyl group. We denote by $s_{i j}$ the transposition $(i j)$ for $1 \leqslant i \neq j \leqslant n$. We have $\mathbb{C}[\mathfrak{t}]=\mathbb{C}\left[x_{1}, \ldots, x_{n}\right]$ and $\mathbb{C}\left[\mathfrak{t}^{*}\right]=\mathbb{C}\left[y_{1}, \ldots, y_{n}\right]$.

We put $\mathfrak{d}(x)=\prod_{i<j}\left(x_{i}-x_{j}\right) \in \mathbb{C}[\mathfrak{t}]$. We denote by $\mathfrak{g}_{\text {reg }}$ the open subset of regular semisimple elements of $\mathfrak{g}$ and we put $\mathfrak{t}_{\text {reg }}=\mathfrak{t} \cap \mathfrak{g}_{\text {reg }}=\{x \in \mathfrak{t} ; \mathfrak{d}(x) \neq 0\}$.

We will identify $\mathbb{C}[\mathfrak{t}]^{W}$ and $\mathbb{C}[\mathfrak{g}]^{G}$ via the restriction map.

Given $M$ a graded vector space, we denote by $M_{k}$ its component of degree $k$.

3.1.2. Let $X$ be a manifold, $i: Y \hookrightarrow X$ a submanifold, and let $f: \mathscr{M} \rightarrow \mathscr{N}$ be a morphism of coherent $\mathscr{D}_{X}$-modules. Assume $Y$ is non-characteristic for $\mathscr{M}$ and $\mathscr{N}$ (i.e., for $Z=$ $\mathrm{Ch}(\mathscr{M})$ or $Z=\mathrm{Ch}(\mathscr{N})$, we have $\left.Z \cap T_{Y}^{*} X \subset T_{X}^{*} X\right)$. If $i^{*}(f): i^{*} \mathscr{M} \rightarrow i^{*} \mathscr{N}$ is an isomorphism (resp. monomorphism, epimorphism), then so is $f$ on a neighbourhood of $Y$ (see e.g. [19, Theorem 4.7]).

3.1.3. Let $f \in H^{0}\left(X ; \mathscr{O}_{X}\right)$ be non zero. We denote by $\delta(f)$ the element $f^{-1}$ of the $\mathscr{D}_{X^{-}}$ module $\mathscr{O}_{X}\left[f^{-1}\right] / \mathscr{O}_{X}$. So, $\mathscr{D}_{X} \delta(f) \subset \mathscr{O}_{X}\left[f^{-1}\right] / \mathscr{O}_{X}$. More generally, let $S$ be a closed subvariety of complete intersection of codimension $r$ given by $f_{1}=\cdots=f_{r}=0$ for $f_{1}, \ldots, f_{r} \in H^{0}\left(X ; \mathscr{O}_{X}\right)$. Then

$$
\mathscr{H}_{S}^{j}\left(\mathscr{O}_{X}\right)=0 \text { for } j \neq r \text { and } \mathscr{H}_{S}^{r}\left(\mathscr{O}_{X}\right) \simeq \mathscr{O}\left[\left(f_{1} \cdots f_{r}\right)^{-1}\right] / \sum_{1 \leqslant i \leqslant r} \mathscr{O}\left[\left(f_{1} \cdots \hat{f}_{i} \cdots f_{r}\right)^{-1}\right]
$$

We denote the last $\mathscr{D}_{X}$-module by $\mathscr{B}_{S \mid X}$. We denote by $\delta\left(f_{1}\right) \cdots \delta\left(f_{r}\right)$ the section $1 /\left(f_{1} \cdots f_{r}\right)$ of $\mathscr{B}_{S \mid X}$.

\subsection{Construction of some $\mathscr{D}$-modules.}


3.2.1. Given $c \in \mathbb{C}$, we denote by $H_{c}$ the rational Cherednik algebra of $(\mathfrak{t}, W)$ with parameter $c$ : this is the $\mathbb{C}$-algebra quotient of $T\left(\mathfrak{t}^{*} \oplus \mathfrak{t}\right) \rtimes W$ by the relations

$$
\begin{aligned}
& {\left[x_{i}, x_{j}\right]=0, \quad\left[y_{i}, y_{j}\right]=0,} \\
& {\left[y_{i}, x_{j}\right]=c s_{i j} \quad \text { for } i \neq j,} \\
& {\left[y_{i}, x_{i}\right]=1-c \sum_{k \neq i} s_{i k} .}
\end{aligned}
$$

We have a vector space decomposition ("PBW-property") [6, Theorem 1.3]

$$
H_{c}=\mathbb{C}[\mathfrak{t}] \otimes \mathbb{C}\left[\mathfrak{t}^{*}\right] \otimes \mathbb{C}[W] .
$$

There is an injective algebra morphism (given by Dunkl operators) [6, Proposition 4.5]

$$
\theta_{c}: H_{c} \hookrightarrow \mathscr{D}\left(\mathfrak{t}_{\text {reg }}\right) \rtimes W \subset \operatorname{End}_{\mathbb{C}}\left(\mathbb{C}\left[\mathfrak{t}_{\text {reg }}\right]\right)
$$

given by the canonical map on $\mathbb{C}[\mathfrak{t}] \rtimes W$ and by

$$
\theta_{c}\left(y_{i}\right)=\partial_{x_{i}}-c \sum_{k \neq i} \frac{1}{x_{i}-x_{k}}\left(1-s_{i k}\right) .
$$

It induces an isomorphism of algebras after localization

$$
\mathbb{C}\left[\mathfrak{t}_{\mathrm{reg}}\right] \otimes_{\mathbb{C}[\mathfrak{t}]} H_{c} \stackrel{\sim}{\longrightarrow} \mathscr{D}\left(\mathfrak{t}_{\mathrm{reg}}\right) \rtimes W .
$$

We denote by $e:=\frac{1}{n !} \sum_{w \in W} w \in \mathbb{C}[W] \subset H_{c}$ and $e_{\mathrm{det}}:=\frac{1}{n !} \sum_{w \in W} \operatorname{det}(w) w \in \mathbb{C}[W] \subset H_{c}$ the idempotents corresponding to the trivial representation and the sign representation of $W$.

We have an injective morphism $\mathbb{C}[\mathfrak{t}]^{W} \rightarrow e H_{c} e, a \mapsto a e$, and we identify $\mathbb{C}[\mathfrak{t}]^{W}$ with its image. We put $\mathbf{y}^{2}=\sum_{i=1}^{n} y_{i}^{2} \in H_{c}$. Recall that $e H_{c} e$ is generated by $\mathbb{C}[\mathfrak{t}]^{W} e$ and $\mathbb{C}\left[\mathfrak{t}^{*}\right]^{W} e$ (cf. e.g. [4, proof of Proposition 5.4.4]). On the other hand, we have an isomorphism of $\mathbb{C}[W]$-modules (cf. e.g. $[2$, Corollary 4.9])

$$
\left(\operatorname{ad}\left(\mathbf{y}^{2}\right)\right)^{k}: \mathbb{C}[\mathfrak{t}]_{k} \stackrel{\sim}{\longrightarrow}\left[\mathfrak{t}^{*}\right]_{k} .
$$

It sends $a\left(x_{1}, \ldots, x_{n}\right)$ to $2^{k} k ! a\left(y_{1}, \ldots, y_{n}\right)$. Hence $e H_{c} e$ is generated by $\mathbb{C}[\mathfrak{t}]^{W} e$ and $\mathbf{y}^{2} e$.

We denote by $h \mapsto h^{*}$ the anti-involution of $H_{c}$ given by $x_{i} \mapsto x_{i}, y_{i} \mapsto-y_{i}, w \mapsto w^{-1}$ $(w \in W)$.

3.2.2. We will identify $\mathfrak{g}$ and $\mathfrak{g}^{*}$ via the $G$-invariant bilinear symmetric form $\mathfrak{g} \times \mathfrak{g} \ni$ $\left(A, A^{\prime}\right) \mapsto \operatorname{tr}\left(A A^{\prime}\right)$.

A pair $(A, z)$ will denote a point of $\mathfrak{g} \times V$. We identify $T^{*}(\mathfrak{g} \times V)$ with $\mathfrak{g} \times \mathfrak{g} \times V \times V^{*}$, and denote accordingly a point in $T^{*}(\mathfrak{g} \times V)$ by $(A, B, z, \zeta)$. Let $\mu: T^{*}(\mathfrak{g} \times V) \rightarrow \mathfrak{g}^{*}$ be the moment map. It is given by $\mu(A, B, z, \zeta)=-[A, B]-z \circ \zeta$.

Let us denote by

$$
\mu_{D}: \mathfrak{g} \rightarrow \mathscr{D}_{\mathfrak{g} \times V}(\mathfrak{g} \times V)
$$

the Lie algebra homomorphism associated with the diagonal action of $G$ on $\mathfrak{g} \times V$. Let us consider the $\mathscr{D}_{\mathfrak{g} \times V}$-module $\mathscr{L}_{c}=\mathscr{D}_{\mathfrak{g} \times V} u_{c}$ given by the defining equation:

$$
\left(\mu_{D}(C)+c \operatorname{tr}(C)\right) u_{c}=0 \quad(C \in \mathfrak{g}) .
$$

More formally, we have $\mathscr{L}_{c}=\mathscr{D}_{\mathfrak{g} \times V} /\left(\mathscr{D}_{\mathfrak{g} \times V}\left(\mu_{D}+c \operatorname{tr}\right)(\mathfrak{g})\right)$ and $u_{c}$ is the image of 1 in $\mathscr{L}_{c}$. 
We consider $\mathscr{L}_{c}$ as a twisted $G$-equivariant $\mathscr{D}_{\mathfrak{g} \times V}$-module with twist $c$ tr, where $u_{c}$ is a $G$-invariant section of $\mathscr{L}_{c}$. Since any $a \in \mathbb{C}[\mathfrak{g}]^{G}$ commutes with $\mu_{D}(C)(C \in \mathfrak{g})$, the map $u_{c} \mapsto a u_{c}$ extends to a $\mathscr{D}_{\mathfrak{g} \times V}$-linear endomorphism of $\mathscr{L}_{c}$. Hence, $\mathscr{L}_{c}$ has a $\left(\mathbb{C}[\mathfrak{t}]^{W} \otimes \mathscr{D}_{\mathfrak{g} \times V}\right)$ module structure.

The characteristic variety $\mathrm{Ch}\left(\mathscr{L}_{c}\right)$ of $\mathscr{L}_{c}$ is the almost commuting variety:

$$
\operatorname{Ch}\left(\mathscr{L}_{c}\right)=\mu^{-1}(0)=\{(A, B, z, \zeta) ;[A, B]+z \circ \zeta=0\} .
$$

This is a complete intersection in $T^{*}(\mathfrak{g} \times V)$ [7, Theorem 1.1].

Lemma 3.1. Let $\mathfrak{g}_{1}$ be the open subset of $\mathfrak{g}$ of elements which have at least $(n-1)$ distinct eigenvalues. We have

$$
\mathscr{H}_{(\mathfrak{g} \backslash \mathfrak{g} \text { reg }) \times V}^{0}\left(\mathscr{L}_{c}\right)=0 \text { and } \mathscr{H}_{\left(\mathfrak{g} \backslash \mathfrak{g}_{1}\right) \times V}^{1}\left(\mathscr{L}_{c}\right)=0
$$

Proof. Since $\mathrm{Ch}\left(\mathscr{L}_{c}\right)$ is a complete intersection, we have $([19,(2.23)])$

$$
\mathscr{E} x t_{\mathscr{D}_{\mathfrak{g} \times V}}^{j}\left(\mathscr{L}_{c}, \mathscr{D}_{\mathfrak{g} \times V}\right)=0 \text { for } j \neq \operatorname{codim}_{T^{*}(\mathfrak{g} \times V)} \mu^{-1}(0)=n^{2} .
$$

Let $\gamma: \mathfrak{g} \rightarrow \mathfrak{t} / W$ be the canonical map associating to $A \in \mathfrak{g}$ the eigenvalues of $A$. Let $\tilde{\gamma}: \mu^{-1}(0) \rightarrow \mathfrak{t} / W$ be given by $(A, B, i, j) \mapsto \gamma(A)$. Then, $\tilde{\gamma}$ is a flat morphism [7, Corollary 2.7].

Let $S$ be a closed subset of $\mathfrak{t} / W$. Since $\tilde{\gamma}$ is flat, we have

$$
\operatorname{codim}_{T^{*}(\mathfrak{g} \times V)}\left(\gamma^{-1}(S) \times_{\mathfrak{g}} \operatorname{Ch}\left(\mathscr{L}_{c}\right)\right)-\operatorname{codim}_{T^{*}(\mathfrak{g} \times V)} \operatorname{Ch}\left(\mathscr{L}_{c}\right)=\operatorname{codim}_{\mathfrak{t} / W} S .
$$

Lemma 2.1 applied to $\gamma^{-1}(S) \times_{\mathfrak{g}} \mathrm{Ch}\left(\mathscr{L}_{c}\right)$ implies

$$
\mathscr{H}_{\gamma^{-1}(S) \times V}^{j}\left(\mathscr{L}_{c}\right)=0 \quad \text { for } j<\operatorname{codim}_{\mathfrak{t} / W} S
$$

and the lemma follows.

3.2.3. Let us recall some constructions and results of [14]. Let $\mu_{0}: \mathfrak{g} \rightarrow \mathscr{D}_{\mathfrak{t} \times \mathfrak{g}}(\mathfrak{t} \times \mathfrak{g})$ be the morphism given by the action of $G$ on $\mathfrak{t} \times \mathfrak{g}: g \cdot(x, A)=(x, \operatorname{Ad}(g) A)$. We consider the $\mathscr{D}_{\mathfrak{t} \times \mathfrak{g}}$-module generated by $\delta_{0}(x, A)$ with the defining equations:

$$
\begin{aligned}
\mu_{0}(C) \delta_{0}(x, A)=0 & \text { for any } C \in \mathfrak{g}, \\
(P(A)-P(x)) \delta_{0}(x, A) & =0 \\
\left(P\left(\partial_{A}\right)-P\left(-\partial_{x}\right)\right) \delta_{0}(x, A) & =0
\end{aligned} \quad \text { for any } P \in \mathbb{C}[\mathfrak{g}]^{G} .
$$

Then, $\mathscr{D}_{\mathfrak{t} \times \mathfrak{g}} \delta_{0}(x, A)$ is a simple holonomic $\mathscr{D}_{\mathfrak{t} \times \mathfrak{g}}$-module with support $\mathfrak{t} \times_{\mathfrak{t}} / W \mathfrak{g}$. Its characteristic variety is the set of $(x, y, A, B)$ such that $[A, B]=0$ and there exists $g \in$ $G$ such that $\operatorname{Ad}(g) A$ and $\operatorname{Ad}(g) B$ are upper triangular and $x$ and $y$ are the diagonal components of $\operatorname{Ad}(g) A$ and $\operatorname{Ad}(g) B$. Note that $\mathscr{D}_{\mathfrak{t} \times \mathfrak{g}} \delta_{0}(x, A) \subset \mathscr{B}_{\mathfrak{t} \times \mathfrak{t} / W \mathfrak{g} \mid \mathfrak{t} \times \mathfrak{g}}$ by $\delta_{0}(x, A) \mapsto$ $\prod_{i=1}^{n} \delta\left(P_{i}(x)-P_{i}(A)\right)$ (see $\left.\S 3.1 .3\right)$, where $P_{i} \in \mathbb{C}[\mathfrak{g}]^{G}(i=1, \ldots, n)$ are the fundamental invariants given by $\operatorname{det}(1+t A)=\sum_{i=0}^{n} P_{i}(A) t^{i}$.

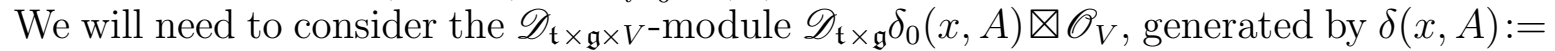
$\delta_{0}(x, A) \otimes 1$ which satisfies the same equations as $\delta_{0}(x, A)$ and $\partial_{z_{i}} \delta(x, A)=0$. In particular, $\mu_{D}(C) \delta(x, A)=0$ for any $C \in \mathfrak{g}$. 
3.2.4. Let us set

$$
q(A, z)=\operatorname{det}\left(A^{n-1} z, A^{n-2} z, \ldots, A z, z\right) .
$$

We have $q(\operatorname{Ad}(g) A, g z)=\operatorname{det}(g) q(A, z)$ for $g \in G$ and $\left[\mu_{D}(C), q(A, z)\right]=-\operatorname{tr}(C) q(A, z)$ for $C \in \mathfrak{g}$.

Consider the $\mathscr{D}_{\mathfrak{t} \times \mathfrak{g} \times V}$-module $\mathscr{D}_{\mathfrak{t} \times \mathfrak{g} \times V} q(A, z)^{c} \delta(x, A)$. A precise definition is as follows. Let us consider the left ideal $\mathscr{I}$ of $\mathscr{D}_{\mathfrak{t} \times \mathfrak{g} \times V} \otimes \mathbb{C}[s]$ ( $s$ being an indeterminate) consisting of those $P(s)$ such that $P(m) q(A, z)^{m} \delta(x, A)=0$ for any $m \in \mathbb{Z}_{\geqslant 0}$. We now define $\mathscr{D}_{\mathfrak{t} \times \mathfrak{g} \times V} q(A, z)^{c} \delta(x, A)$ as $\left(\mathscr{D}_{\mathfrak{t} \times \mathfrak{g} \times V} \otimes \mathbb{C}[s]\right) /\left(\mathscr{I}+\mathscr{D}_{\mathfrak{t} \times \mathfrak{g} \times V} \otimes \mathbb{C}[s](s-c)\right)$. It is a holonomic

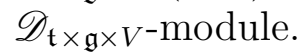

The element $q(A, z)^{c} \delta(x, A)$ satisfies

$$
\begin{aligned}
& \left(\mu_{D}(C)+c \operatorname{tr}(C)\right) q(A, z)^{c} \delta(x, A)=0 \quad \text { for any } C \in \mathfrak{g}, \\
& (P(A)-P(x)) q(A, z)^{c} \delta(x, A)=0 \quad \text { for any } P \in \mathbb{C}[\mathfrak{g}]^{G} .
\end{aligned}
$$

We put $v_{c}=q(A, z)^{c} \delta(x, A)$. Let $p_{0}: \mathfrak{t}_{\text {reg }} \times \mathfrak{g} \times V \rightarrow \mathfrak{g} \times V$ be the projection. Let us consider the $\mathscr{D}_{\mathfrak{g} \times V^{-m o d u l e}}$

$$
\mathscr{M}_{c}=\left(p_{0}\right)_{*}\left(\mathscr{D}_{\mathfrak{t}_{\mathrm{reg}} \times \mathfrak{g} \times V} v_{c}\right)=\left(p_{0}\right)_{*}\left(\mathscr{D}_{\mathrm{t}_{\mathrm{reg}} \times \mathfrak{g} \times V} q(A, z)^{c} \delta(x, A)\right) .
$$

By the definition, we have an isomorphism $\mathscr{M}_{c} \stackrel{\sim}{\longrightarrow} j_{*} j^{-1} \mathscr{M}_{c}$ where $j: \mathfrak{g}_{\text {reg }} \times V \hookrightarrow \mathfrak{g} \times V$ is the open embedding. This is a quasi-coherent $\mathscr{D}_{\mathfrak{g} \times V}$-module whose characteristic variety is contained in the almost commuting variety $\mu^{-1}(0)$.

The action of $W$ on $\mathfrak{t}_{\text {reg }}$ induces a $W$-action on $\mathscr{M}_{c}$. Here, $W$ acts trivially on $v_{c}$. Hence, the $\mathscr{D}_{\mathfrak{g} \times V}$-module $\mathscr{M}_{c}$ has a module structure over $\mathscr{D}\left(\mathfrak{t}_{\text {reg }}\right) \rtimes W$. Therefore, $H_{c}$ acts on $\mathscr{M}_{c}$ via the canonical embedding $\theta_{c}: H_{c} \hookrightarrow \mathscr{D}\left(\mathfrak{t}_{\text {reg }}\right) \rtimes W$.

\subsection{Spherical constructions and shift.}

3.3.1. There is a $\mathscr{D}_{\mathfrak{g} \times V^{-}}$-linear homomorphism

$$
\iota: \mathscr{L}_{c} \rightarrow \mathscr{M}_{c}, u_{c} \mapsto v_{c}
$$

We regard $\mathscr{M}_{c}$ as a twisted $G$-equivariant $\mathscr{D}_{\mathfrak{g} \times V}$-module with twist $c$ tr, where sections in $\mathscr{D}\left(\mathfrak{t}_{\text {reg }}\right) v_{c}$ are $G$-invariant. Then, the morphism above is $G$-equivariant. Moreover, it is $\mathbb{C}[\mathfrak{t}]^{W}$-linear. Hence $\iota$ induces an epimorphism of $\left(\mathscr{D}\left(\mathfrak{t}_{\text {reg }}\right) \rtimes W\right) \otimes \mathscr{D}_{\mathfrak{g} \times V}$-modules:

$$
\mathscr{D}\left(\mathfrak{t}_{\text {reg }}\right) \otimes_{\mathbb{C}[\mathfrak{t}]^{W}} \mathscr{L}_{c} \rightarrow \mathscr{M}_{c}
$$

Lemma 3.2. The morphism of $\mathbb{C}[W] \otimes \mathscr{D}_{\mathfrak{g} \times V}$-modules

$$
1 \otimes \iota: \mathbb{C}[\mathfrak{t}] \otimes_{\mathbb{C}[\mathfrak{t}]^{W}} \mathscr{L}_{c} \rightarrow \mathscr{M}_{c}
$$

is an isomorphism on $\mathfrak{g}_{\mathrm{reg}} \times V$.

In particular, the induced morphisms $\mathscr{L}_{c} \stackrel{u_{c} \mapsto v_{c}}{\longrightarrow} e \mathscr{M}_{c}$ and $\mathscr{L}_{c} \stackrel{u_{c} \mapsto \mathfrak{o}(x) v_{c}}{\longrightarrow} e_{\operatorname{det}} \mathscr{M}_{c}$ are isomorphisms on $\mathfrak{g}_{\mathrm{reg}} \times V$.

Proof. Let $i: \mathfrak{t}_{\text {reg }} \times V \hookrightarrow \mathfrak{g} \times V$ be the embedding. Note that $i$ is non-characteristic for $\mathscr{L}_{c}$ and $\mathscr{M}_{c}$. Since $G \cdot \mathfrak{t}_{\text {reg }}=\mathfrak{g}_{\text {reg }}$, it is enough to prove that the canonical map $\mathbb{C}\left[\mathfrak{t}_{\text {reg }}\right] \otimes_{\mathbb{C}\left[\mathfrak{t}_{\text {reg }}\right]^{W}} i^{*} \mathscr{L}_{c} \rightarrow i^{*} \mathscr{M}_{c}$ is an isomorphism (cf. $\S 3.1 .2$ ).

We have $i^{*} \mu_{D}\left(e_{r s}\right)=\left(A_{r r}-A_{s s}\right) \partial_{A_{r s}}-z_{s} \partial_{z_{r}}$. It follows that we have an isomorphism

$$
\mathscr{D}_{\mathfrak{t}_{\text {reg }} \times V} /\left(\sum_{i} \mathscr{D}_{\mathfrak{t}_{\text {reg }} \times V}\left(z_{i} \partial_{z_{i}}-c\right)\right) \stackrel{\sim}{\sim} i^{*} \mathscr{L}_{c}, 1 \mapsto i^{*} u_{c}
$$


Let $i^{\prime \prime}: \mathfrak{t}_{\text {reg }} \times \mathfrak{t}_{\text {reg }} \hookrightarrow \mathfrak{t} \times \mathfrak{g}$ be the embedding. Since the Jacobian

$$
\partial\left(P_{1}(x), \ldots, P_{n}(x)\right) / \partial\left(x_{1}, \ldots, x_{n}\right)
$$

is equal to $\mathfrak{d}(x)$ (e.g. [5, Ch. V, $\S 5.4$, Proposition 5]), we have an isomorphism

$$
i^{\prime \prime *} \mathscr{D}_{\mathfrak{t} \times \mathfrak{g}} \delta_{0}(x, A) \frac{\delta_{0}(x, A) \mapsto \sum_{w} \mathfrak{d}(a)^{-1} \delta\left(w^{-1} x-a\right)}{\sim} \bigoplus_{w \in W} \mathscr{D}_{\mathfrak{t}_{\mathrm{reg}} \times \mathfrak{t}_{\mathrm{reg}}} \delta\left(w^{-1} x-a\right)
$$

where $\delta\left(w^{-1} x-a\right)=\delta\left(x_{w(1)}-a_{1}\right) \cdots \delta\left(x_{w(n)}-a_{n}\right)$.

Let us denote by $i^{\prime}: \mathfrak{t}_{\text {reg }} \times \mathfrak{t}_{\text {reg }} \times V \hookrightarrow \mathfrak{t}_{\text {reg }} \times \mathfrak{g} \times V$ the embedding. We have an isomorphism

$$
i^{\prime *} \mathscr{D}_{\mathfrak{t}_{\text {reg }} \times \mathfrak{g} \times V} v_{c} \stackrel{v_{c} \mapsto \sum_{w} v_{w}^{\prime}}{\sim} \bigoplus_{w \in W} \mathscr{D}_{\mathfrak{t}_{\text {reg }} \times \mathfrak{t}_{\text {reg }} \times V} v_{w}^{\prime} .
$$

where $v_{w}^{\prime}=\mathfrak{d}(a)^{c-1}\left(z_{1} \cdots z_{n}\right)^{c} \delta\left(w^{-1} x-a\right)$ has the defining equations

$$
\begin{aligned}
& \left(\partial_{x_{w(i)}}+\partial_{a_{i}}-(c-1) \sum_{j \neq i} \frac{1}{a_{i}-a_{j}}\right) v_{w}^{\prime}=0 \\
& \left(x_{w(i)}-a_{i}\right) v_{w}^{\prime}=0 \\
& \left(z_{i} \partial_{z_{i}}-c\right) v_{w}^{\prime}=0
\end{aligned}
$$$$
\text { for any } i=1, \ldots, n \text {. }
$$

In particular, we have

$$
f(x) v_{w}^{\prime}=\left(w^{-1} f\right)(a) v_{w}^{\prime} \quad \text { for any } f \in \mathbb{C}[\mathfrak{t}] .
$$

We obtain finally an isomorphism

$$
i^{*} \mathscr{M}_{c} \stackrel{v_{c} \mapsto \sum_{w} v_{w}^{\prime}}{\sim} \bigoplus_{w \in W} \mathscr{D}_{\mathfrak{t}_{\mathrm{reg}} \times V} v_{w}^{\prime} .
$$

This is compatible with the action of $W$, where $w^{\prime}\left(v_{w}^{\prime}\right)=v_{w^{\prime} w}^{\prime}$. Moreover, each $\mathscr{D}_{\mathfrak{t}_{\mathrm{reg} \times V} v_{w}^{\prime}}$ is isomorphic to $i^{*} \mathscr{L}_{c}$ by $v_{w}^{\prime} \mapsto u_{c}$. Hence we obtain an isomorphism of $\left(\mathscr{D}_{\mathfrak{t}_{\text {reg }} \times V} \otimes \mathbb{C}[W]\right)$ modules

$$
i^{*} \mathscr{M}_{c} \stackrel{\sim}{\longrightarrow}[W] \otimes i^{*} \mathscr{L}_{c}
$$

The composition $i^{*}\left(\mathbb{C}[\mathfrak{t}] \otimes_{\mathbb{C}[\mathfrak{t}]^{W}} \mathscr{L}_{c}\right) \rightarrow i^{*} \mathscr{M}_{c} \stackrel{\sim}{\longrightarrow} \mathbb{C}[W] \otimes i^{*} \mathscr{L}_{c}$ is given by $a \otimes u_{c} \mapsto$ $\sum_{w \in W} w \otimes\left(w^{-1} a\right) u_{c}$ in virtue of (3.6). Then the lemma follows from the fact that $\mathbb{C}[\mathfrak{t}] \otimes_{\mathbb{C}[\mathfrak{t}]^{W}} \mathbb{C}\left[\mathfrak{t}_{\text {reg }}\right] \rightarrow \mathbb{C}[W] \otimes \mathbb{C}\left[\mathfrak{t}_{\text {reg }}\right]$ given by $a \otimes b \mapsto \sum_{w \in W} w \otimes\left(w^{-1} a\right) b$ is an isomorphism.

Lemma 3.3. The morphism $\iota: \mathscr{L}_{c} \rightarrow \mathscr{M}_{c}$ is injective and its image is stable by e $H_{c} e$. Furthermore, e $H_{c}$ e acts faithfully on $\mathscr{L}_{c}$.

Proof. The injectivity of $\iota$ follows from Lemma 3.2, because $\mathscr{L}_{c}$ does not have a non-zero submodule supported in $\left(\mathfrak{g} \backslash \mathfrak{g}_{\text {reg }}\right) \times V$ by Lemma 3.1 .

Since $e H_{c} e$ is generated by $\mathbb{C}[\mathfrak{t}]^{W}$ and $\mathbf{y}^{2} e(\mathrm{cf} . \S 3.2 .1$ ), the stability result follows from the following result (cf. [4, Proposition 5.4.1] and [6, Proposition 6.2]):

$$
\mathbf{y}^{2} v_{c}=\Delta_{\mathfrak{g}} v_{c} \text {. }
$$

Here $\Delta_{\mathfrak{g}}=\sum_{i, j=1, \ldots, n} \frac{\partial^{2}}{\partial A_{i j} \partial A_{j i}}$ is the Laplacian on $\mathfrak{g}$. 
Finally, the faithfulness of the action of $e H_{c} e$ follows from the faithfulness of the action of $H_{c}$ on $H_{c} v_{c} \subset \mathscr{M}_{c}$. With the notations of the proof of Lemma 3.2, we have an isomorphism $i^{*} \mathscr{M}_{c} \simeq \mathscr{D}_{\mathfrak{t}_{\text {reg }} \times V} \rtimes W$ compatible with the action of $\mathscr{D}_{\mathfrak{t}_{\text {reg }}} \rtimes W$, and the faithfulness follows from that of $\theta_{c}$.

Remark 3.4. (i) In other words, the subalgebra of End $\mathscr{\mathscr { D }}_{\mathfrak{g} \times V}\left(\mathscr{L}_{c}\right)$ generated by $\mathbb{C}[\mathfrak{t}]^{W}$ and by the endomorphism $u_{c} \mapsto \Delta_{\mathfrak{g}} u_{c}$ is isomorphic to $e H_{c} e$.

(ii) The action of $e H_{c} e$ on $\mathscr{L}_{c}$ can be described as follows. Let $\kappa_{0}: \mathbb{C}[\mathfrak{t}]^{W} \stackrel{\sim}{\sim}[\mathfrak{g}]^{G} \hookrightarrow$ $\mathscr{D}(\mathfrak{g})$ and $\kappa_{1}: \mathbb{C}\left[\mathfrak{t}^{*}\right]^{W} \stackrel{\sim}{\sim}\left[\mathfrak{g}^{*}\right]^{G} \hookrightarrow \mathscr{D}(\mathfrak{g})$ be the canonical morphisms. We have

$$
\begin{aligned}
& \text { (ae) } u_{c}=\kappa_{0}(a) u_{c} \quad \text { for } a \in \mathbb{C}[\mathfrak{t}]^{W}, \\
& \text { (be) } u_{c}=\kappa_{1}\left(b^{*}\right) u_{c} \quad \text { for } b \in \mathbb{C}\left[\mathfrak{t}^{*}\right]^{W} .
\end{aligned}
$$

The first equality is clear. We have a commutative diagram

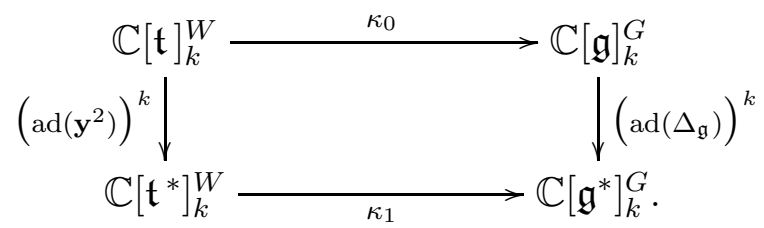

From (3.7) and the first equality, we deduce that

$$
\left(\operatorname{ad}\left(\Delta_{\mathfrak{g}}\right)\right)^{k}\left(\kappa_{0}(a)\right) v_{c}=(-1)^{k}\left(\operatorname{ad}\left(\mathbf{y}^{2}\right)\right)^{k}(a) v_{c}
$$

for $a \in \mathbb{C}[\mathfrak{t}]_{k}^{W}$. This gives the second equality.

3.3.2. The morphism $\iota$ gives rise to an $\left(H_{c} \otimes \mathscr{D}_{\mathfrak{g} \times V}\right)$-linear morphism

$$
H_{c} e \otimes_{e H_{c} e} \mathscr{L}_{c} \rightarrow \mathscr{M}_{c} .
$$

Consider the conditions:

$$
\begin{aligned}
& H_{c} e H_{c}=H_{c} \\
& e H_{c} e_{\operatorname{det}} H_{c} e=e H_{c} e \text { and } e_{\operatorname{det}} H_{c} e H_{c} e_{\operatorname{det}}=e_{\operatorname{det}} H_{c} e_{\operatorname{det}} .
\end{aligned}
$$

Lemma 3.5. If (3.11) is satisfied, then the morphism (3.10) is injective.

Proof. Since $H_{c} e$ is a projective $e H_{c} e$-module, any coherent submodule of $H_{c} e \otimes_{e H_{c} e} \mathscr{L}_{c}$ vanishes as soon as it is zero on $\mathfrak{g}_{\mathrm{reg}} \times V$ by Lemma 3.1. Hence it is enough to show that the morphism (3.10) is injective on $\mathfrak{g}_{\mathrm{reg}} \times V$. Then the result follows from Lemma 3.2 and the fact that the multiplication map gives an isomorphism of right $\left(e H_{c} e \otimes_{\mathbb{C}[\mathfrak{t}]^{W}} \mathbb{C}\left[\mathfrak{t}_{\text {reg }}\right]^{W}\right)$ modules

$$
\mathbb{C}[\mathfrak{t}] \otimes_{\mathbb{C}[\mathfrak{t}]^{W}} e H_{c} e \otimes_{\mathbb{C}[\mathfrak{t}]^{W}} \mathbb{C}\left[\mathfrak{t}_{\mathrm{reg}}\right]^{W} \stackrel{\sim}{\sim} H_{c} e \otimes_{\mathbb{C}[\mathfrak{t}]^{W}} \mathbb{C}\left[\mathfrak{t}_{\mathrm{reg}}\right]^{W}
$$

Proposition 3.6. Condition (3.11) holds if and only if e $H_{c}$ gives a Morita equivalence between $H_{c}$ and $e H_{c}$ e. Similarly, Condition (3.12) holds if and only if $e H_{c} e_{\operatorname{det}}$ gives a Morita equivalence between $e_{\operatorname{det}} H_{c} e_{\operatorname{det}}$ and $\mathrm{eH}_{c} e$.

This follows from the following Lemma:

Lemma 3.7. Let $A$ be a ring, and let $e_{1}$ and $e_{2}$ be idempotents in $A$. Assume that

$$
e_{1} A e_{2} A e_{1}=e_{1} A e_{1} \quad \text { and } e_{2} A e_{1} A e_{2}=e_{2} A e_{2} .
$$


(i) For any A-module $M$, we have

$$
e_{2} A e_{1} \otimes_{e_{1} A e_{1}} e_{1} M \stackrel{\sim}{\sim} e_{2} M .
$$

(ii) $e_{1} A e_{2}$ and $e_{2} A e_{1}$ give a Morita equivalence between $\operatorname{Mod}\left(e_{1} A e_{1}\right)$ and $\operatorname{Mod}\left(e_{2} A e_{2}\right)$.

Proof. (i) The surjectivity follows from $e_{2} M=e_{2} A e_{2} M=e_{2} A e_{1} A e_{2} M \subset\left(e_{2} A e_{1}\right)\left(e_{1} M\right)$.

Let us show its injectivity. By the assumption, there exists finitely many elements $a_{i} \in e_{2} A e_{1}$ and $b_{i} \in e_{1} A e_{2}$ such that $e_{2}=\sum_{i} a_{i} b_{i}$. Consider now $u=\sum_{j} x_{j} \otimes v_{j} \in$ $e_{2} A e_{1} \otimes_{e_{1} A e_{1}} e_{1} M$ (where $x_{j} \in e_{2} A e_{1}, v_{j} \in e_{1} M$ ). Assume $\sum_{j} x_{j} v_{j}=0$. Then

$$
u=\sum_{j, i} a_{i} b_{i} x_{j} \otimes v_{j}=\sum_{j, i} a_{i} \otimes b_{i} x_{j} v_{j}=0 .
$$

(ii) It is enough to show that the multiplication maps $e_{2} A e_{1} \otimes_{e_{1} A e_{1}} e_{1} A e_{2} \rightarrow e_{2} A e_{2}$ and $e_{1} A e_{2} \otimes_{e_{2} A e_{2}} e_{2} A e_{1} \rightarrow e_{1} A e_{1}$ are isomorphisms. For the first one, we apply (i) to $M=A e_{2}$. The second one can be handled similarly.

The previous result can be expressed in terms of bimodules:

Proposition 3.8. Let $A$ and $B$ be rings, and let $P$ be an $(A, B)$-bimodule, $Q$ a $(B, A)$ bimodule and let $\varphi: P \otimes_{B} Q \rightarrow A$ be a morphism of $(A, A)$-bimodules, and $\psi: Q \otimes_{A} P \rightarrow$ $B$ a morphism of $(B, B)$-bimodules. Assume that $\varphi$ and $\psi$ are surjective and that the following diagrams commute:

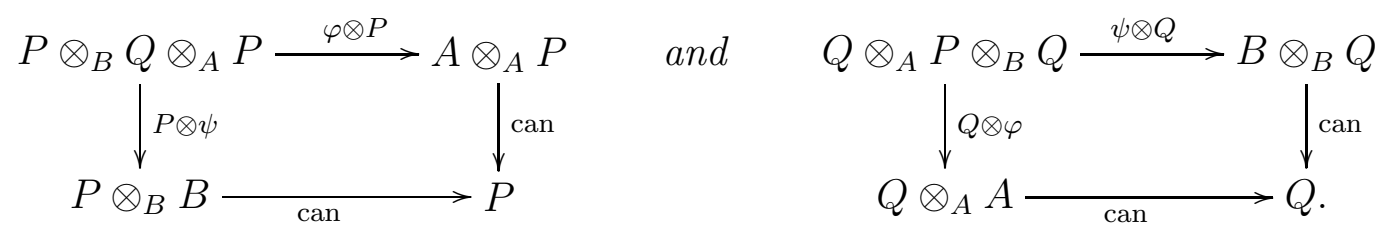

(i) Then $\varphi$ and $\psi$ are isomorphisms, and $P$ and $Q$ give a Morita equivalence between $\operatorname{Mod}(A)$ and $\operatorname{Mod}(B)$.

(ii) Let $M$ be an $A$-module and $N$ a $B$-module, and let $f: Q \otimes_{A} M \rightarrow N$ and $g: P \otimes_{B}$ $N \rightarrow M$ be morphisms such that the diagrams

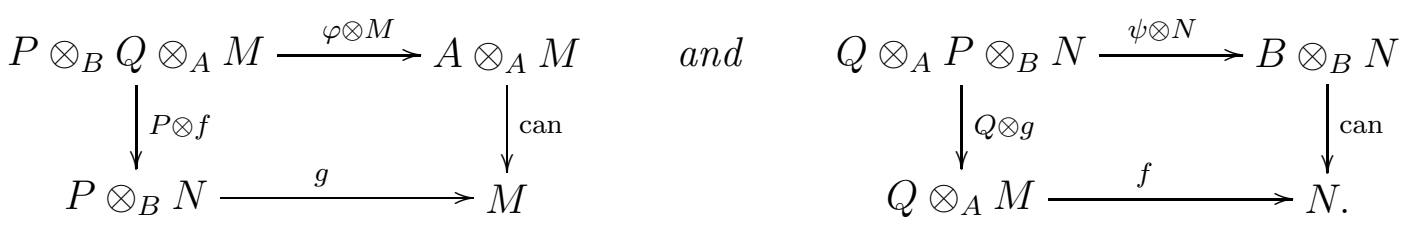

are commutative. Then $f$ and $g$ are isomorphisms.

Proof. Apply Lemma 3.7 to the $\operatorname{ring}\left(\begin{array}{ll}A & P \\ Q & B\end{array}\right)$, its module $\left(\begin{array}{c}M \\ N\end{array}\right)$ and $e_{1}=\left(\begin{array}{ll}1 & 0 \\ 0 & 0\end{array}\right)$ and $e_{2}=\left(\begin{array}{ll}0 & 0 \\ 0 & 1\end{array}\right)$.

Remark 3.9. (i) It would be interesting to describe the image of the morphism $(3.10)$.

(ii) Let

$$
\mathscr{Y}=\left\{\frac{m}{d} \mid m, d \in \mathbb{Z}, 2 \leqslant d \leqslant n,(m, d)=1, m<0\right\} .
$$


It is known that Condition (3.11) holds for $c \notin \mathscr{Y}$, while Condition (3.12) holds when $c-1 \notin \mathscr{Y}$, cf. [8, Theorem 3.3], [2, Theorem 8.1] and [3].

3.3.3. Let us consider the $\mathscr{D}\left(\mathfrak{t}_{\text {reg }}\right) \otimes \mathscr{D}_{\mathfrak{g} \times V}$-linear morphism

$$
\begin{aligned}
\sigma: \mathscr{M}_{c} & \rightarrow \mathscr{M}_{c-1} \otimes \operatorname{det}(V) \\
v_{c}=q(A, z)^{c} \delta(x, A) & \mapsto q(A, z) \cdot q(A, z)^{c-1} \delta(x, A) \otimes l=q(A, z) v_{c-1} \otimes l .
\end{aligned}
$$

Here $l \in \operatorname{det}(V):=\bigwedge^{n} V$ is the element such that $q(A, z) l=A^{n-1} z \wedge A^{n-2} z \wedge \cdots \wedge A z \wedge z$. In particular, $q(A, z) \otimes l$ is a $G$-invariant section of $\mathscr{O}_{\mathfrak{g} \times V} \otimes \operatorname{det}(V)$.

So, the morphism $\sigma$ is $G$-equivariant. We endow $\mathscr{M}_{c-1}$ with an $H_{c}$-module structure via the embedding $\theta_{c}: H_{c} \hookrightarrow \mathscr{D}\left(\mathfrak{t}_{\text {reg }}\right) \rtimes W$. Then $\sigma$ is $H_{c}$-linear.

Remark 3.10. Note that $\mathscr{M}_{c} \rightarrow \mathscr{M}_{c-1} \otimes \operatorname{det}(V)$ is an isomorphism on $\{q(A, z) \neq 0\}$. However, with our definition of $\mathscr{M}_{c}$, the morphism $\mathscr{M}_{c} \rightarrow \mathscr{M}_{c-1} \otimes \operatorname{det}(V)$ is not a monomorphism for certain $c$, e.g. $c=0$. Let us show this after restriction to $\mathfrak{t}_{\text {reg }} \times V$. We have $q\left({ }^{t} A, \partial_{z}\right) q(A, z) v_{c-1}=0$ for $c=0$ by (3.5), while the support of $q\left({ }^{t} A, \partial_{z}\right) v_{c}$ is the subvariety $\{q(A, z)=0\}$.

Let $\mathscr{D}_{\mathfrak{g} \times V}\left(\mathfrak{d}(x) v_{c-1}\right)$ be the $\mathscr{D}_{\mathfrak{g} \times V}$-submodule of $\mathscr{M}_{c-1}$ generated by $\mathfrak{d}(x) v_{c-1}$.

Lemma 3.11. (i) $\mathscr{D}_{\mathfrak{g} \times V}\left(\mathfrak{d}(x) v_{c-1}\right)$ is invariant by $e_{\operatorname{det}} H_{c} e_{\operatorname{det}}$.

(ii) The morphism $\mathscr{L}_{c-1} \rightarrow \mathscr{D}_{\mathfrak{g} \times V}\left(\mathfrak{d}(x) v_{c-1}\right)$ given by $u_{c-1} \mapsto \mathfrak{d}(x) v_{c-1}$ is an isomorphism.

Proof. Note that $e_{\operatorname{det}} \mathfrak{d}(x) v_{c-1}=\mathfrak{d}(x) v_{c-1}$. The proof is similar to that of Lemma 3.3: the key point is the following (cf. e.g. [13, Theorem 3.1])

$$
\mathbf{y}^{2}\left(\mathfrak{d}(x) v_{c-1}\right)=\Delta_{\mathfrak{g}}\left(\mathfrak{d}(x) v_{c-1}\right) .
$$

By [2, Proposition 4.1], there is a (unique) isomorphism

$$
f: e_{\mathrm{det}} H_{c} e_{\mathrm{det}} \stackrel{\sim}{\sim} e H_{c-1} e
$$

such that $\theta_{c-1}(f(a))=\mathfrak{d}(x)^{-1} \theta_{c}(a) \mathfrak{d}(x)$ for $a \in e_{\mathrm{det}} H_{c} e_{\mathrm{det}}$.

The isomorphism $\mathscr{L}_{c-1} \stackrel{\sim}{\longrightarrow} \mathscr{D}_{\mathfrak{g} \times V}\left(\mathfrak{d}(x) v_{c-1}\right)$ of Lemma 3.11 is compatible with $f$ and we will sometimes view $\mathscr{L}_{c-1}$ as an $\left(e_{\operatorname{det}} H_{c} e_{\operatorname{det}} \otimes \mathscr{D}_{\mathfrak{g} \times V}\right)$-module.

By Lemma 3.2, the image of the morphism

$$
\left.\left.e_{\mathrm{det}} H_{c} e \otimes_{e H_{c} e} \mathscr{L}_{c}\right|_{\mathfrak{g}_{\mathrm{reg} \times V}} \rightarrow \mathscr{M}_{c}\right|_{\mathfrak{g}_{\mathrm{reg} \times V}}, a \otimes u_{c} \mapsto a v_{c}
$$

is contained in $\mathscr{D}_{\mathfrak{g}_{\mathrm{reg}} \times V}\left(\mathfrak{d}(x) v_{c}\right)$. It follows from Lemma 3.11 that over $\mathfrak{g}_{\text {reg }} \times V$, the composite morphism $e_{\operatorname{det}} H_{c} e \otimes_{e H_{c} e} \mathscr{L}_{c} \rightarrow \mathscr{M}_{c} \rightarrow \mathscr{M}_{c-1} \otimes \operatorname{det}(V)$ factors through a morphism

$$
\varphi:\left.\left.e_{\mathrm{det}} H_{c} e \otimes_{e H_{c} e} \mathscr{L}_{c}\right|_{\mathfrak{g}_{\mathrm{reg}} \times V} \longrightarrow \mathscr{L}_{c-1} \otimes \operatorname{det}(V)\right|_{\mathfrak{g}_{\mathrm{reg}} \times V} .
$$

Similarly, we have the morphism

$$
\begin{aligned}
\psi:\left.e H_{c} e_{\mathrm{det}} \otimes_{e_{\mathrm{det}} H_{c} e_{\mathrm{det}}} \mathscr{L}_{c-1} \otimes \operatorname{det}(V)\right|_{\{q(A, z) \neq 0\}} & \left.\rightarrow \mathscr{L}_{c}\right|_{\{q(A, z) \neq 0\}} \\
a \otimes u_{c-1} \otimes l & \mapsto(a \mathfrak{d}(x)) q(A, z)^{-1} u_{c} .
\end{aligned}
$$

The morphism $\varphi$ is linear over $e_{\mathrm{det}} H_{c} e_{\mathrm{det}} \simeq e H_{c-1} e$ and the morphism $\psi$ is linear over $e H_{c} e$. We have

$$
\varphi\left(\mathfrak{d}(x) e \otimes u_{c}\right)=q(A, z) u_{c-1} \otimes l
$$


and

$$
q(A, z) \psi\left(\mathfrak{d}(x) e_{\operatorname{det}} \otimes u_{c-1} \otimes l\right)=\mathfrak{d}^{2}(A) u_{c}
$$

where $\mathfrak{d}^{2}(A)$ is the discriminant of the characteristic polynomial of $A$.

Note that the following diagrams commute on $\mathfrak{g}_{\mathrm{reg}} \times V \cap\{q(A, z) \neq 0\}$ :

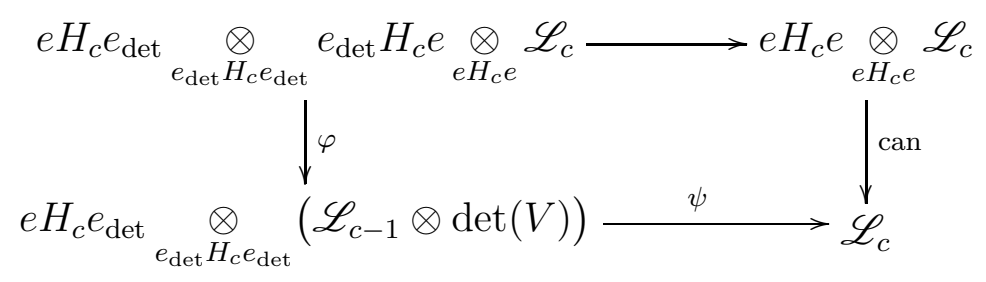

and

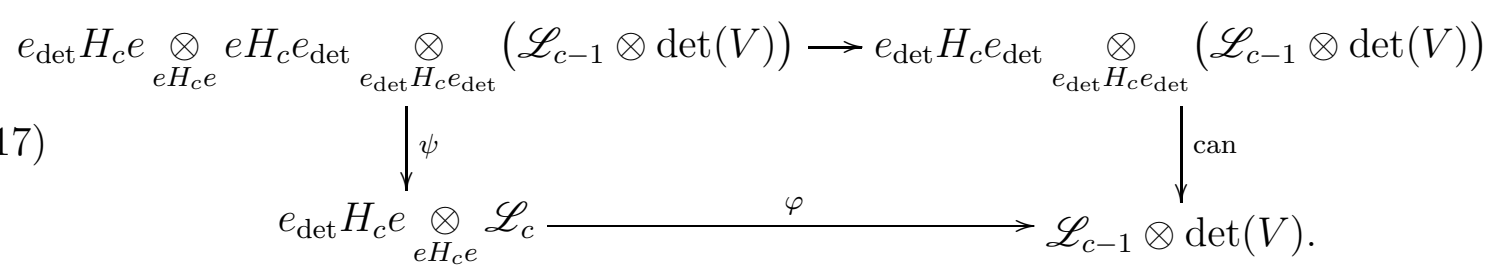

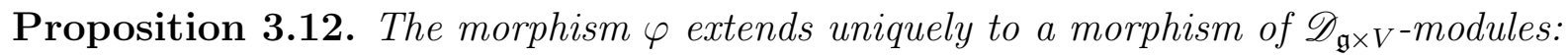

$$
\varphi: e_{\operatorname{det}} H_{c} e \otimes_{e H_{c} e} \mathscr{L}_{c} \longrightarrow \mathscr{L}_{c-1} \otimes \operatorname{det}(V) .
$$

The proof will proceed by reduction to rank two. Recall that $\mathfrak{g}_{1}$ denotes the open subset of $\mathfrak{g}$ of matrices with at least $(n-1)$ distinct eigenvalues. Then $\mathfrak{g} \backslash \mathfrak{g}_{1}$ is a closed subset of $\mathfrak{g}$ of codimension 2 .

We shall prove first the following lemma.

Lemma 3.13. After restriction to $\mathfrak{g}_{1} \times V$, we have an inclusion of submodules of $\mathscr{M}_{c-1}$

$$
H_{c} \mathscr{D}_{\mathfrak{g} \times V} \bar{v}_{c} \subset \mathbb{C}[\mathfrak{t}] \mathscr{D}_{\mathfrak{g} \times V} \bar{v}_{c}+\mathbb{C}[\mathfrak{t}] \mathscr{D}_{\mathfrak{g} \times V} \mathfrak{d}(x) v_{c-1}
$$

where $\bar{v}_{c}=q(A, z) v_{c-1}$.

Proof. Since $H_{c}=\mathbb{C}[\mathfrak{t}] \mathbb{C}\left[\mathfrak{t}^{*}\right] \mathbb{C}[W]$, it is enough to show that

$$
\mathbb{C}\left[\mathfrak{t}^{*}\right] \mathscr{D}_{\mathfrak{g} \times V} \bar{v}_{c} \in \mathbb{C}[\mathfrak{t}] \mathscr{D}_{\mathfrak{g} \times V} \bar{v}_{c}+\mathbb{C}[\mathfrak{t}] \mathscr{D}_{\mathfrak{g} \times V} \mathfrak{d}(x) v_{c-1} \quad \text { on } \mathfrak{g}_{1} \times V .
$$

Here the action of $\mathbb{C}\left[\mathfrak{t}^{*}\right]$ is through $\mathbb{C}\left[\mathfrak{t}^{*}\right] \hookrightarrow H_{c} \stackrel{\theta_{c}}{\longrightarrow} \mathscr{D}\left(\mathfrak{t}_{\text {reg }}\right) \rtimes W$.

Let us assume first that $n=2$. We have

$$
q(A, z)=-A_{21} z_{1}^{2}+\left(A_{11}-A_{22}\right) z_{1} z_{2}+A_{12} z_{2}^{2} .
$$

We put

$$
q\left(\partial_{A}, z\right)=-z_{1}^{2} \partial_{A_{12}}+z_{1} z_{2}\left(\partial_{A_{11}}-\partial_{A_{22}}\right)+z_{2}^{2} \partial_{A_{21}}
$$

We will show that

$$
\left(\partial_{x_{1}}-\partial_{x_{2}}\right) q(A, z) v_{c-1}=-q\left(\partial_{A}, z\right)\left(x_{1}-x_{2}\right) v_{c-1} .
$$

This is an equality in the $\mathscr{D}_{\mathfrak{g} \times V^{-}}$submodule $\iota\left(\mathscr{L}_{c-1}\right)$ of $\mathscr{M}_{c-1}$. Note that $\left(y_{1}-y_{2}\right) v_{c-1}=$ $\left(\partial_{x_{1}}-\partial_{x_{2}}\right) v_{c-1}$.

By $\S 3.2 .3$, we have

$$
v_{c-1}=q(A, z)^{c-1} \delta\left(x_{1}+x_{2}-\operatorname{tr}(A)\right) \delta\left(x_{1} x_{2}-\operatorname{det}(A)\right) .
$$


Since $q\left(\partial_{A}, z\right) q(A, z)=q\left(\partial_{A}, z\right) \operatorname{tr}(A)=0$ and $q\left(\partial_{A}, z\right) \operatorname{det}(A)=-q(A, z)$, we obtain

$$
q\left(\partial_{A}, z\right) v_{c-1}=q(A, z)^{c} \delta\left(x_{1}+x_{2}-\operatorname{tr}(A)\right) \delta^{\prime}\left(x_{1} x_{2}-\operatorname{det}(A)\right) .
$$

On the other hand, we have

$$
\left(\partial_{x_{1}}-\partial_{x_{2}}\right) q(A, z) v_{c-1}=\left(x_{2}-x_{1}\right) q(A, z)^{c} \delta\left(x_{1}+x_{2}-\operatorname{tr}(A)\right) \delta^{\prime}\left(x_{1} x_{2}-\operatorname{det}(A)\right) .
$$

The equality (3.20) then follows.

We assume now $n \geqslant 2$. Let $S$ be the locally closed subset of $\mathfrak{g}$ of matrices

$$
\left(\begin{array}{ccccc}
A^{\prime} & 0 & 0 & \ldots & \\
0 & a_{3} & 0 & \ldots & \\
0 & 0 & a_{4} & & \\
\vdots & \vdots & & \ddots & \\
& & & & a_{n}
\end{array}\right)
$$

where $A^{\prime}$ is a $2 \times 2$ matrix and $a_{i} \neq a_{j}(3 \leqslant i<j \leqslant n)$ and $a_{i}$ is not an eigenvalue of $A^{\prime}$ for $3 \leqslant i \leqslant n$. Let $\mathfrak{t}_{1}=\mathfrak{t} \cap S=\left\{x \in \mathfrak{t} ; x_{i} \neq x_{j}\right.$ for $i<j$ and $\left.3 \leqslant j\right\}$. Let $x^{\prime}=\left(x_{1}, x_{2}\right)$, $x^{\prime \prime}=\left(x_{3}, \ldots, x_{n}\right)$ and $a^{\prime \prime}=\left(a_{3}, \ldots, a_{n}\right)$.

We have $G \cdot S=\mathfrak{g}_{1}$. Let $i: S \times V \hookrightarrow \mathfrak{g} \times V$ be the inclusion map. Then, $i$ is noncharacteristic for $\mathscr{L}_{c}$ and $\mathscr{M}_{c-1}$, because we have $T_{x} S+T_{x}(G \cdot x)=T_{x} \mathfrak{g}$ for any $x \in S$.

Denote by $\mathfrak{g}^{\prime}$ the subalgebra of $\mathfrak{g}$ of matrices $\left(A_{i j}\right)$ with $A_{i j}=0$ whenever $i>2$ or $j>2$. We identify $\mathfrak{g}^{\prime}$ with $\mathfrak{g l}_{2}(\mathbb{C})$. Given an object $\mathcal{X}$ defined earlier for $\mathfrak{g}$, we denote by $\mathcal{X}^{\prime}$ the corresponding objects for $\mathfrak{g}^{\prime}$ (i.e., case $n=2$ ). For example, $W^{\prime}$ is the subgroup of $W$ generated by $s_{12}$.

Let $i^{\prime \prime}: \mathfrak{t} \times S \rightarrow \mathfrak{t} \times \mathfrak{g}$ be the embedding. We have an isomorphism of $\mathscr{D}_{\mathfrak{t} \times S^{- \text {modules }}}$ compatible with the action of $W$ (cf. Proof of Lemma 3.2):

$$
i^{\prime \prime *} \mathscr{D}_{\mathfrak{t} \times \mathfrak{g}} \delta(x, A) \frac{\delta(x, A) \mapsto \sum_{w} T_{w}^{*} \mathfrak{d}_{1}\left(A^{\prime}, a^{\prime \prime}\right)^{-1} \delta\left(x^{\prime}, A^{\prime}\right) \delta\left(x^{\prime \prime}-a^{\prime \prime}\right)}{\sim} \bigoplus_{w \in W^{\prime} \backslash W} T_{w}^{*} \mathscr{D}_{\mathfrak{t} \times S} \delta\left(x^{\prime}, A^{\prime}\right) \delta\left(x^{\prime \prime}-a^{\prime \prime}\right) .
$$

Here, $T_{w}$ is the automorphism of $\mathfrak{t}$ given by $w$, and $\mathfrak{d}_{1}\left(A^{\prime}, a^{\prime \prime}\right)=\mathfrak{d}\left(a^{\prime \prime}\right) \prod_{i=3}^{n} \operatorname{det}\left(a_{i} I_{2}-A^{\prime}\right)$, $\delta\left(x^{\prime}, A^{\prime}\right)=\delta\left(x_{1}+x_{2}-\operatorname{tr}\left(A^{\prime}\right)\right) \delta\left(x_{1} x_{2}-\operatorname{det}\left(A^{\prime}\right)\right)$.

Let $A \in S$. We have

where

$$
q(A, z)=q^{\prime}\left(A^{\prime}, z^{\prime}\right) \cdot q_{1}(A, z)
$$

$$
q_{1}(A, z)=\left(z_{3} \cdots z_{n}\right) \mathfrak{d}_{1}\left(A^{\prime}, a^{\prime \prime}\right)
$$

Note that $\mathfrak{d}_{1}\left(A^{\prime}, a^{\prime \prime}\right)$ is invertible on $S$.

Let $p: \mathfrak{t}_{\text {reg }} \times S \times V \rightarrow S \times V$ be the projection. We have a $\mathscr{D}\left(\mathfrak{t}_{\text {reg }}\right) \otimes \mathscr{D}_{S \times V^{-}}$-linear isomorphism compatible with the action of $W$ :

$$
i^{*} \mathscr{M}_{c} \underset{\sim}{\stackrel{v_{c} \mapsto e \otimes \widetilde{v}_{c}}{\longrightarrow}} \mathbb{C}[W] \otimes_{\mathbb{C}\left[W^{\prime}\right]} p_{*}\left(\mathscr{D}_{\mathrm{t}_{\mathrm{reg}} \times S \times V} \widetilde{v}_{c}\right)
$$

where $\widetilde{v}_{c}=v_{c}^{\prime} q_{1}(A, z)^{c} \mathfrak{d}_{1}\left(A^{\prime}, a^{\prime \prime}\right)^{-1} \delta\left(x^{\prime \prime}-a^{\prime \prime}\right)$ with $v_{c}^{\prime}=q^{\prime}\left(A^{\prime}, z^{\prime}\right)^{c} \delta\left(x^{\prime}, A^{\prime}\right)$. Note that $s_{12}$ acts trivially on $\widetilde{v}_{c}$. The action of $\mathscr{D}\left(\mathfrak{t}_{\text {reg }}\right) \rtimes W$ on $\mathbb{C}[W] \otimes_{\mathbb{C}\left[W^{\prime}\right]} p_{*}\left(\mathscr{D}_{\mathfrak{t}_{\text {reg }} \times S \times V} \widetilde{v}_{c}\right)$ is given by:

$(a \otimes w)\left(w^{\prime} \otimes s\right)=\left(w w^{\prime}\right) \otimes\left(\left(\left(w w^{\prime}\right)^{-1} a\right) s\right) \quad$ for $w, w^{\prime} \in W, a \in \mathscr{D}\left(\mathfrak{t}_{\mathrm{reg}}\right), s \in p_{*}\left(\mathscr{D}_{\mathbf{t}_{\mathrm{reg} \times S \times V}} \widetilde{v}_{c}\right)$. Note that $\mathscr{D}_{S \times V} \widetilde{v}_{c}$ is stable by $\mathbb{C}\left[\mathfrak{t}_{1}\right]^{W^{\prime}}$ as a submodule of $p_{*}\left(\mathscr{D}_{\mathfrak{t}_{\mathrm{reg}} \times S \times V} \widetilde{v}_{c}\right)$. Since $\mathbb{C}\left[\mathfrak{t}_{1}\right]=$ $\mathbb{C}[\mathfrak{t}] \mathbb{C}\left[\mathfrak{t}_{1}\right]^{W^{\prime}}, \mathbb{C}[\mathfrak{t}] \mathscr{D}_{S \times V} \widetilde{v}_{c}$ is stable by $\mathbb{C}\left[\mathfrak{t}_{1}\right]$. 
Let us still denote by $\widetilde{v}_{c}=q(A, z) \widetilde{v}_{c-1}$, the image of $\widetilde{v}_{c}$.

Let us set $\widetilde{y}_{1}=\partial_{x_{1}}-c\left(x_{1}-x_{2}\right)^{-1}\left(1-s_{12}\right)$ and $\widetilde{y}_{2}=\partial_{x_{2}}-c\left(x_{2}-x_{1}\right)^{-1}\left(1-s_{12}\right)$, partial Dunkl operators, and let $R$ be the algebra generated by $\widetilde{y}_{1}, \widetilde{y}_{2}$ and $\partial_{x_{i}}(i=3, \ldots, n)$. Then $s_{12}$ acts on $R$ by the permutation of $\widetilde{y}_{1}$ and $\widetilde{y}_{2}$. We have $R=R^{W^{\prime}} \oplus\left(\widetilde{y}_{1}-\widetilde{y}_{2}\right) R^{W^{\prime}}$.

Let

$$
\begin{aligned}
\tilde{\mathscr{N}} & =\mathbb{C}[\mathfrak{t}] \mathscr{D}_{S \times V} \widetilde{v}_{c}+\left(\widetilde{y}_{1}-\widetilde{y}_{2}\right) \mathbb{C}[\mathfrak{t}] \mathscr{D}_{S \times V} \widetilde{v}_{c} \\
& =\mathbb{C}[\mathfrak{t}] \mathscr{D}_{S \times V} \widetilde{v}_{c}+\mathbb{C}[\mathfrak{t}] \mathscr{D}_{S \times V}\left(\widetilde{y}_{1}-\widetilde{y}_{2}\right) \widetilde{v}_{c} \\
& =\mathbb{C}[\mathfrak{t}] \mathscr{D}_{S \times V} \widetilde{v}_{c}+\mathbb{C}[\mathfrak{t}] \mathscr{D}_{S \times V}\left(\partial_{x_{1}}-\partial_{x_{2}}\right) \widetilde{v}_{c}
\end{aligned}
$$

be a submodule of $p_{*}\left(\mathscr{D}_{\mathfrak{t}_{\text {reg }} \times S \times V} \widetilde{v}_{c-1}\right)$. Since $\left(\widetilde{y}_{1}+\widetilde{y}_{2}\right) \widetilde{v}_{c}, \widetilde{y}_{1} \widetilde{y}_{2} \widetilde{v}_{c}$, and $\partial_{x_{i}} \widetilde{v}_{c}(i=3, \ldots, n)$ belong to $\mathbb{C}[\mathfrak{t}] \mathscr{D}_{S \times V} \widetilde{v}_{c}$ (cf. Lemma 3.3), $\tilde{\mathscr{N}}$ is invariant by $R$.

Set $\mathscr{N}=\mathbb{C}[W] \otimes_{\mathbb{C}\left[W^{\prime}\right]} \tilde{\mathscr{N}}$. Let us show that $\mathscr{N}$ is invariant by the action of $\mathbb{C}\left[\mathfrak{t}^{*}\right] \subset$ $H_{c} \subset \mathscr{D}\left(\mathfrak{t}_{\text {reg }}\right) \rtimes W$. For any $i$, we have

$$
y_{i}(w \otimes t)=w \otimes \partial_{x_{w^{-1}(i)}} t-c \sum_{k \neq i} w\left(1+s_{w^{-1}(i), w^{-1}(k)}\right) \otimes\left(x_{w^{-1}(i)}-x_{w^{-1}(k)}\right)^{-1} t
$$

for any $w \in W$ and $t \in \tilde{\mathscr{N}}$. Since $\left(x_{a}-x_{b}\right)^{-1} \in \mathbb{C}\left[\mathfrak{t}_{1}\right]$ when $a$ or $b$ is in $\{3, \ldots, n\}$, we have $y_{i}(w \otimes t) \in \mathscr{N}$ when $w^{-1}(i) \neq 1,2$. If $w^{-1}(i)=1$, then

$$
\begin{aligned}
y_{i}(w \otimes t) & \equiv w \otimes \partial_{x_{1}} t-c w\left(1+s_{12}\right) \otimes\left(x_{1}-x_{2}\right)^{-1} t \quad \bmod \mathscr{N} \\
& =w \otimes \widetilde{y}_{1} t \in \mathscr{N} .
\end{aligned}
$$

The case $w^{-1}(i)=2$ is similar. Hence we have shown that $\mathscr{N}$ is invariant by $\mathbb{C}\left[\mathfrak{t}^{*}\right]$. Thus, we obtain

$$
\mathbb{C}\left[\mathfrak{t}^{*}\right]\left(e \otimes \widetilde{v}_{c}\right) \subset \mathscr{N}
$$

The study of rank 2 above, i.e. (3.20), shows that

$$
\left(\widetilde{y}_{1}-\widetilde{y}_{2}\right) \widetilde{v}_{c} \subset \mathbb{C}[\mathfrak{t}] \mathscr{D}_{S \times V} \widetilde{v}_{c}+\mathbb{C}[\mathfrak{t}] \mathscr{D}_{S \times V}\left(x_{1}-x_{2}\right) \widetilde{v}_{c-1} .
$$

Hence we obtain

$$
\tilde{\mathscr{N}} \subset \tilde{\mathscr{N}}^{\prime}:=\mathbb{C}[\mathfrak{t}] \mathscr{D}_{S \times V} \widetilde{v}_{c}+\mathbb{C}[\mathfrak{t}] \mathscr{D}_{S \times V} \mathfrak{d}(x) \widetilde{v}_{c-1},
$$

which implies

$$
\mathbb{C}\left[\mathfrak{t}^{*}\right]\left(e \otimes \widetilde{v}_{c}\right) \subset \mathscr{N}^{\prime}:=\mathbb{C}[W] \otimes \tilde{\mathscr{N}}^{\prime} .
$$

We have a commutative diagram, where the horizontal map is an isomorphism

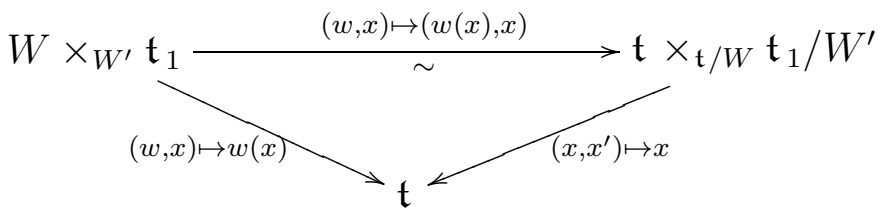

The diagram above is $W$-equivariant, for the action of $g \in W$ given by

$$
\begin{gathered}
g \cdot(w, x)=(g w, x) \text { for }(w, x) \in W \times_{W^{\prime}} \mathfrak{t}_{1} \\
g \cdot\left(x, x^{\prime}\right)=\left(g(x), x^{\prime}\right) \text { for }\left(x, x^{\prime}\right) \in \mathfrak{t} \times_{\mathfrak{t} / W} \mathfrak{t}_{1} / W^{\prime} .
\end{gathered}
$$


It follows that we have an isomorphism of $\mathbb{C}[\mathfrak{t}]$-modules

$$
\begin{aligned}
\mathbb{C}[\mathfrak{t}] \otimes_{\mathbb{C}[\mathfrak{t}]^{W}} \mathbb{C}\left[\mathfrak{t}_{1}\right]^{W^{\prime}} & \stackrel{\sim}{\sim}[W] \otimes_{\mathbb{C}\left[W^{\prime}\right]} \mathbb{C}\left[\mathfrak{t}_{1}\right] \\
a \otimes a^{\prime} & \mapsto \sum_{w \in W / W^{\prime}} w \otimes w^{-1}(a) a^{\prime} .
\end{aligned}
$$

In particular, we have $\mathbb{C}[W] \otimes_{\mathbb{C}\left[W^{\prime}\right]} \mathbb{C}\left[\mathfrak{t}_{1}\right]=\mathbb{C}[\mathfrak{t}] \cdot\left(e \otimes \mathbb{C}\left[\mathfrak{t}_{1}\right]^{W^{\prime}}\right)$. Since $\mathbb{C}\left[\mathfrak{t}_{1}\right]^{W^{\prime}} \widetilde{v}_{c} \subset \mathscr{D}_{S \times V} \widetilde{v}_{c}$ and $\mathbb{C}\left[\mathfrak{t}_{1}\right]^{W^{\prime}} \mathfrak{d}(x) \widetilde{v}_{c-1} \subset \mathscr{D}_{S \times V} \mathfrak{d}(x) \widetilde{v}_{c-1}$, we deduce that

$$
\mathscr{N}^{\prime}=\mathbb{C}[\mathfrak{t}]\left(e \otimes \mathscr{D}_{S \times V} \widetilde{v}_{c}+e \otimes \mathscr{D}_{S \times V} \mathfrak{d}(x) \widetilde{v}_{c-1}\right) .
$$

Together with (3.22), we obtain

$$
\mathbb{C}\left[\mathfrak{t}^{*}\right] \mathscr{D}_{S \times V}\left(e \otimes \widetilde{v}_{c}\right) \subset \mathbb{C}[\mathfrak{t}]\left(\mathscr{D}_{S \times V}\left(e \otimes \widetilde{v}_{c}\right)+\mathscr{D}_{S \times V}\left(e \otimes \mathfrak{d}(x) \widetilde{v}_{c-1}\right)\right)
$$

Via the isomorphism (3.21), this shows that

$$
i^{*}\left(\mathbb{C}\left[\mathfrak{t}^{*}\right] \mathscr{D}_{\mathfrak{g} \times V} \bar{v}_{c}\right) \subset i^{*}\left(\mathbb{C}[\mathfrak{t}] \mathscr{D}_{\mathfrak{g} \times V} \bar{v}_{c}+\mathbb{C}[\mathfrak{t}] \mathscr{D}_{\mathfrak{g} \times V} \mathfrak{d}(x) v_{c-1}\right)
$$

Since $\mu^{-1}(0) \cap T_{S \times V}^{*}(\mathfrak{g} \times V) \subset T_{\mathfrak{g} \times V}^{*}(\mathfrak{g} \times V)$, the non-characteristic condition implies the desired result (3.19) (cf. $\S 3.1 .2$ ).

Proof of Proposition 3.12. By Lemma 3.13, we have, on $\mathfrak{g}_{1} \times V$,

$$
\begin{aligned}
e_{\operatorname{det}} H_{c} \mathscr{D}_{\mathfrak{g} \times V} \bar{v}_{c} & \subset e_{\operatorname{det}} \mathbb{C}[\mathfrak{t}] \mathscr{D}_{\mathfrak{g} \times V} \bar{v}_{c}+e_{\operatorname{det}} \mathbb{C}[\mathfrak{t}] \mathscr{D}_{\mathfrak{g} \times V} \mathfrak{d}(x) v_{c-1} \\
& \subset \mathbb{C}[\mathfrak{t}]^{W} \mathfrak{d}(x) \mathscr{D}_{\mathfrak{g} \times V} \bar{v}_{c}+\mathbb{C}[\mathfrak{t}]^{W} \mathscr{D}_{\mathfrak{g} \times V} \mathfrak{d}(x) v_{c-1}=\mathscr{D}_{\mathfrak{g} \times V} \mathfrak{d}(x) v_{c-1},
\end{aligned}
$$

since $e_{\operatorname{det}} \mathbb{C}[\mathfrak{t}] e=\mathbb{C}[\mathfrak{t}]^{W} \mathfrak{d}(x) e$ and $e_{\operatorname{det}} \mathbb{C}[\mathfrak{t}] e_{\operatorname{det}}=\mathbb{C}[\mathfrak{t}]^{W} e_{\operatorname{det}}$. Hence $\varphi$ extends to a mor-

phism defined on $\mathfrak{g}_{1} \times V$. Then the desired result follows from $\mathscr{H}_{\left(\mathfrak{g} \backslash \mathfrak{g}_{1}\right) \times V}^{1}\left(\mathscr{L}_{c-1}\right)=0$ (Lemma 3.1).

\section{Cherednik algebras And Hilbert schemes}

\subsection{Geometry of the Hilbert scheme.}

4.1.1. We refer to $[23,12]$ for basic results on Hilbert schemes of points on $\mathbb{C}^{2}$.

Let us recall that

$$
\mathfrak{X}=\left\{(A, B, z, \zeta) \in \mathfrak{g} \times \mathfrak{g} \times V \times V^{*} ; \mathbb{C}\langle A, B\rangle z=V\right\}
$$

is the set of stable points for the action of $G$ on $T^{*}(\mathfrak{g} \times V)$, relative to the character det of $G$. The group $G$ acts freely on $\mathfrak{X}$. Let $\mu_{\mathfrak{X}}: \mathfrak{X} \rightarrow \mathfrak{g}$ be the moment map:

$$
\mu_{\mathfrak{X}}(A, B, z, \zeta)=-[A, B]-z \circ \zeta .
$$

It is a smooth morphism. Let $\operatorname{Hilb}^{n}\left(\mathbb{C}^{2}\right)$ be the Hilbert scheme classifying closed subschemes of $\mathbb{C}^{2}$ with length $n$. Then we have an isomorphism $\operatorname{Hilb}^{n}\left(\mathbb{C}^{2}\right) \stackrel{\sim}{\longrightarrow} \mu_{\mathfrak{X}}^{-1}(0) / G$. Note that we have $\zeta=0$ on $\mu_{\mathfrak{X}}^{-1}(0)$ (cf. [7, Lemma 2.3]).

We shall write Hilb instead of $\operatorname{Hilb}^{n}\left(\mathbb{C}^{2}\right)$ for short. Let us denote by $p: \mu_{\mathfrak{X}}^{-1}(0) \rightarrow$ Hilb the quotient map.

Let us recall the construction of $p$. For $(A, B, z, \zeta) \in \mu_{\mathfrak{X}}^{-1}(0)$, we regard $V$ as a $\mathbb{C}[X, Y]$ module by $X \mapsto A$ and $Y \mapsto B$. Then $z$ gives an epimorphism $\mathbb{C}[X, Y] \rightarrow V$ of $\mathbb{C}[X, Y]$ modules. Hence $V$ gives a closed subscheme of $\mathbb{C}^{2}=\operatorname{Spec}(\mathbb{C}[X, Y])$ of length $n$, which is the corresponding point of Hilb. 
Let $\pi:$ Hilb $\rightarrow\left(\mathfrak{t} \times \mathfrak{t}^{*}\right) / W$ be the Hilbert-Chow morphism. Then Hilb is a resolution of singularities of $\left(\mathfrak{t} \times \mathfrak{t}^{*}\right) / W \simeq\left(\mathbb{C}^{2}\right)^{n} / S_{n}$, the scheme of $n$ unordered points in $\mathbb{C}^{2}$. We have canonical isomorphisms

$$
\Gamma\left(\mu_{\mathfrak{X}}^{-1}(0), \mathscr{O}_{\mu_{\mathfrak{X}}^{-1}(0)}\right)^{G} \stackrel{\sim}{\longrightarrow} \Gamma\left(\text { Hilb }, \mathscr{O}_{\text {Hilb }}\right) \stackrel{\sim}{\longrightarrow} \Gamma\left(\left(\mathfrak{t} \times \mathfrak{t}^{*}\right) / W, \mathscr{O}_{\left(\mathfrak{t} \times \mathfrak{t}^{*}\right) / W}\right) \stackrel{\sim}{\longrightarrow}\left[\mathfrak{C} \times \mathfrak{t}^{*}\right]^{W} .
$$

Let $\left(\mathfrak{t} \times \mathfrak{t}^{*}\right)_{\text {reg }}$ be the open subset of $\mathfrak{t} \times \mathfrak{t}^{*}$ where the action of $W$ is free. The Hilbert-Chow morphism $\pi$ is an isomorphism over $\left(\mathfrak{t} \times \mathfrak{t}^{*}\right)_{\text {reg }} / W$. Let $E:=\pi^{-1}\left(\left(\left(\mathfrak{t} \times \mathfrak{t}^{*}\right) \backslash\left(\mathfrak{t} \times \mathfrak{t}^{*}\right)_{\mathrm{reg}}\right) / W\right)$ be the exceptional divisor. It is a closed irreducible hypersurface of Hilb. The line bundle $L$ on Hilb associated with the $G$-equivariant line bundle $\mathscr{O}_{\mathfrak{X}} \otimes \operatorname{det}(V)$ on $\mathfrak{X}$ is a very ample line bundle on Hilb.

Let us set

$$
\mathbb{C}\left[\mu_{\mathfrak{X}}^{-1}(0)\right]^{G, \operatorname{det}}=\left\{\phi(p) \in \mathbb{C}\left[\mu_{\mathfrak{X}}^{-1}(0)\right] ; \phi(g p)=\operatorname{det}(g) \phi(p) \text { for any } g \in G\right\} .
$$

It is isomorphic to $\Gamma($ Hilb,$L) \simeq\left(\mathbb{C}\left[\mu_{\mathfrak{X}}^{-1}(0)\right] \otimes \operatorname{det}(V)\right)^{G}$. Let $i: \mathfrak{t} \times \mathfrak{t}^{*} \times V \hookrightarrow \mathfrak{g} \times \mathfrak{g} \times V \times V^{*}$ be the embedding with the last component $\zeta=0$. Then $i^{-1}\left(\mu_{\mathfrak{X}}^{-1}(0)\right)$ contains $\left(\mathfrak{t}_{\text {reg }} \times \mathfrak{t}^{*} \cup\right.$ $\left.\mathfrak{t} \times \mathfrak{t}_{\text {reg }}^{*}\right) \times\left(\mathbb{C}^{*}\right)^{n}$. For any $\phi \in \mathbb{C}\left[\mu_{\mathfrak{X}}^{-1}(0)\right]^{G \text {,det }}$, we have $\left(i^{*} \phi\right)(x, y, g z)=\operatorname{det}(g)\left(i^{*} \phi\right)(x, y, z)$ for any invertible diagonal matrix $g$. Hence we have

$$
\left(i^{*} \phi\right)(x, y, z)=a(x, y)\left(z_{1} \cdots z_{n}\right)
$$

for some rational function $a(x, y)$ which is regular on $\left(\mathfrak{t}_{\text {reg }} \times \mathfrak{t}^{*}\right) \cup\left(\mathfrak{t} \times \mathfrak{t}_{\text {reg }}^{*}\right)$, an open subset of $\mathfrak{t} \times \mathfrak{t}^{*}$ with complement of codimension 2. Hence we have

$$
a(x, y) \in \mathbb{C}\left[\mathfrak{t} \times \mathfrak{t}^{*}\right]^{W, \operatorname{det}}=\left\{a \in \mathbb{C}\left[\mathfrak{t} \times \mathfrak{t}^{*}\right] ; w a=\operatorname{det}(w) a \text { for any } w \in W\right\} .
$$

Thus we obtain a map which is known to be an isomorphism (cf. e.g. [7, Proposition 8.2.1]) and we denote its inverse by $i_{d}$ :

$$
\begin{aligned}
\mathbb{C}\left[\mu_{\mathfrak{X}}^{-1}(0)\right]^{G, \operatorname{det}} \otimes \operatorname{det}(V) & \stackrel{\sim}{\longrightarrow}\left[\mathfrak{t} \times \mathfrak{t}^{*}\right]^{W, \operatorname{det}} \\
\phi \otimes l & \mapsto\left\langle l, z_{1} \wedge \cdots \wedge z_{n}\right\rangle a .
\end{aligned}
$$

Similarly, we have an isomorphism (cf. e.g. [7, Lemma 2.7.3]) whose inverse we denote by $i_{s}$ :

$$
\mathbb{C}\left[\mu_{\mathfrak{X}}^{-1}(0)\right]^{G} \stackrel{\sim}{\longrightarrow}\left[\mathfrak{t} \times \mathfrak{t}^{*}\right]^{W}
$$

Summarizing, we have the following isomorphisms

$$
\begin{aligned}
\left.i_{d}: \mathbb{C}_{\mathfrak{t}} \times \mathfrak{t}^{*}\right]^{W, \operatorname{det}} \stackrel{\sim}{\sim} \mathbb{C}\left[\mu_{\mathfrak{X}}^{-1}(0)\right]^{G, \operatorname{det}} \otimes \operatorname{det}(V) & \simeq \Gamma(\text { Hilb }, L), \\
i_{s}: \mathbb{C}\left[\mathfrak{t} \times \mathfrak{t}^{*}\right]^{W} \stackrel{\sim}{\longrightarrow} \mathbb{C}\left[\mu_{\mathfrak{X}}^{-1}(0)\right]^{G} & \simeq \mathscr{O}_{\text {Hilb }}(\text { Hilb }) .
\end{aligned}
$$

4.1.2. For a subset $Y$ of $\mathbb{Z}_{\geqslant 0} \times \mathbb{Z}_{\geqslant 0}$ with cardinality $n$, set $p_{Y}=\operatorname{det}\left(x_{k}^{i} y_{k}^{j}\right)_{(i, j) \in Y, k=1, \ldots n} \in$ $\mathbb{C}\left[\mathfrak{t} \times \mathfrak{t}^{*}\right]^{W \text {,det }}$ and $s_{Y}(A, B, z, \zeta)=\operatorname{det}\left(A^{i} B^{j} z\right)_{(i, j) \in Y} \in \mathbb{C}\left[\mu_{\mathfrak{X}}^{-1}(0)\right]^{G \text {,det }}=L($ Hilb $)$. Then $\left\{p_{Y}\right\}_{Y}$ is a basis of $\mathbb{C}\left[\mathfrak{t} \times \mathfrak{t}^{*}\right]^{W \text {,det }}$ as a vector space and $i_{d}\left(p_{Y}\right)=s_{Y}$. The $\mathscr{O}_{\text {Hilb-module }}$ $L$ is generated by $\left\{s_{Y}\right\}_{Y}$, where $Y$ ranges over the set of Young diagrams of size $n$. Here we regard a Young diagram $Y$ as a subset of $\mathbb{Z}_{\geqslant 0} \times \mathbb{Z}_{\geqslant 0}$ such that $(i, j) \in Y$ as soon as $(i, j+1)$ or $(i+1, j)$ belongs to $Y$.

There is a canonical global section $\tau \in \Gamma\left(\mathrm{Hilb} ; L^{\otimes-2}\right)$ satisfying the following property:

$$
i_{d}\left(a_{1}\right) i_{d}\left(a_{2}\right) \tau=i_{s}\left(a_{1} a_{2}\right) \quad \text { for any } a_{1}, a_{2} \in \mathbb{C}\left[\mathfrak{t} \times \mathfrak{t}^{*}\right]^{W, \operatorname{det}} .
$$


Note that $\tau$ is identified with a function on $\mu_{\mathfrak{X}}^{-1}(0)$ such that $\tau(g p)=\operatorname{det}(g)^{-2} \tau(p)(p \in$ $\mu_{\mathfrak{X}}^{-1}(0)$ and $\left.g \in G\right)$.

The exceptional divisor $E$ coincides with the set of zeroes of $\tau$, and we obtain an isomorphism

$$
L^{\otimes 2} \stackrel{\sim}{\longrightarrow} \mathscr{O}_{\mathrm{Hilb}}(-E) .
$$

Let us denote by $\mathfrak{d}^{2}(A)$ the discriminant of the characteristic polynomial of $A$, and similarly for $\mathfrak{d}^{2}(B)$. Then we have

$$
i_{d}(\mathfrak{d}(x))=q(A, z), \quad i_{d}(\mathfrak{d}(y))=q(B, z), \quad i_{s}\left(\mathfrak{d}(x)^{2}\right)=\mathfrak{d}^{2}(A), \quad i_{s}\left(\mathfrak{d}(y)^{2}\right)=\mathfrak{d}^{2}(B) .
$$

Hence we have

$$
\mathfrak{d}^{2}(A)=q(A, z)^{2} \tau \text { and } \mathfrak{d}^{2}(B)=q(B, z)^{2} \tau .
$$

Lemma 4.1. (i) The hypersurface of $\mu_{\mathfrak{X}}^{-1}(0)$ defined by $q(A, z)=0$ is irreducible, and $p^{-1} E \cap\{q(A, z)=0\}$ is of codimension 2 in $\mu_{\mathfrak{X}}^{-1}(0)$.

(ii) The hypersurface of $\mu_{\mathfrak{X}}^{-1}(0)$ defined by $\mathfrak{d}^{2}(A)=0$ is $p^{-1} E \cup\{q(A, z)=0\}$.

(iii) $\mu_{\mathfrak{X}}^{-1}(0) \cap\{q(A, z)=q(B, z)=0\}$ is of codimension 2 in $\mu_{\mathfrak{X}}^{-1}(0)$.

Note that (i) follows from the fact that $q(A, z)$ does not vanish on the irreducible hypersurface $p^{-1} E$ of $\mu_{\mathfrak{X}}^{-1}(0)$, and $q(A, z)$ is irreducible on $\mu_{\mathfrak{X}}^{-1}(0) \backslash p^{-1} E$. Statement (iii) follows from [12, Lemma 3.6.2].

\subsection{W-algebras on the Hilbert scheme.}

4.2.1. In the preceding sections, we have regarded $\mathfrak{X}$, Hilb, etc. as schemes. Hereafter, we regard them as complex manifolds. Note that the previous constructions and results would remain valid in the analytic category. Let $\mathscr{W}_{\mathfrak{X}}$ be the $\mathscr{W}$-algebra on $\mathfrak{X}$ associated with $\mathscr{D}_{\mathfrak{g} \times V}$. Denoting by $\pi: \mathfrak{X} \rightarrow \mathfrak{g} \times V$ the projection, we have a ring homomorphism $\pi^{-1} \mathscr{D}_{\mathfrak{g} \times V} \rightarrow \mathscr{W}_{\mathfrak{X}}$ respecting the order filtration. The ring $\mathscr{W}_{\mathfrak{X}}$ is flat over $\pi^{-1} \mathscr{D}_{\mathfrak{g} \times V}$. The action of $G$ on $\mathfrak{g} \times V$ induces an action of $G$ on $\mathscr{W}_{\mathfrak{X}}$ and there is a quantized moment map $\mu_{\mathscr{W}}: \mathfrak{g} \rightarrow \mathscr{W}_{\mathfrak{X}}$.

We have morphisms

$$
\kappa_{0}: \mathbb{C}[\mathfrak{t}]^{W} \stackrel{\sim}{\longrightarrow}[\mathfrak{g}]^{G} \rightarrow \mathscr{W}_{\mathfrak{X}}(\mathfrak{X})
$$

and

$$
\kappa_{1}: \mathbb{C}\left[\mathfrak{t}^{*}\right]^{W} \stackrel{\sim}{\longrightarrow}\left[\mathfrak{g}^{*}\right]^{G} \hookrightarrow \mathscr{D}_{\mathfrak{g}}(\mathfrak{g}) \rightarrow \mathscr{W}_{\mathfrak{X}}(\mathfrak{X}) .
$$

Note that $\kappa_{1}\left(\mathbf{y}^{2}\right)=\Delta_{\mathfrak{g}}$.

For $k \in \mathbb{Z}_{\geqslant 0}$, let $\mathbb{C}\left[\mathfrak{t}^{*}\right]_{k}^{W}$ be the homogeneous part of $\mathbb{C}\left[\mathfrak{t}^{*}\right]^{W}$ of degree $k$. Then $\kappa_{0}$ sends $\mathbb{C}[\mathfrak{t}]^{W}$ to $\mathscr{W}_{\mathfrak{X}}(0)$ and $\kappa_{1}$ sends $\mathbb{C}\left[\mathfrak{t}^{*}\right]_{k}^{W}$ to $\mathscr{W}_{\mathfrak{X}}(k)$ and we have the commutative diagrams:

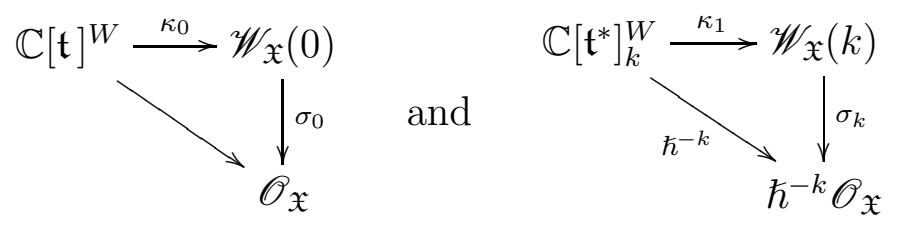

Let us consider $\mathscr{W}_{\mathfrak{X}} \otimes_{\mathscr{D}_{\mathfrak{g} \times V}} \mathscr{L}_{c}$, which we denote by the same letter $\mathscr{L}_{c}$. With the notation of $\S 2.4 .2$, we have $\mathscr{L}_{c}=\Phi_{c \text { tr }}\left(\mathscr{W}_{\mathfrak{X}}\right)$. Hence $\mathscr{L}_{c}$ is a twisted $G$-equivariant $\mathscr{W}_{\mathfrak{X}}$-module with twist $c$ tr. Let $u_{c}$ be the canonical section of $\mathscr{L}_{c}$, and set $\mathscr{L}_{c}(m)=\mathscr{W}_{\mathfrak{X}}(m) u_{c}$. Then we have an isomorphism

$$
\mathscr{L}_{c}(0) / \mathscr{L}_{c}(-1) \stackrel{\sim}{\longrightarrow} \mathscr{O}_{\mu_{\mathfrak{X}}^{-1}(0)} .
$$


The support of $\mathscr{L}_{c}$ is $\mu_{\mathfrak{X}}^{-1}(0)$. The $\mathscr{W}_{\mathfrak{X}}$-module $\mathscr{L}_{c}$ has a left action of $e H_{c} e$ by Lemma 3.3. Via the anti-involution $h \mapsto h^{*}$ of $H_{c}$, we regard $\mathscr{L}_{c}$ as a $\left(\mathscr{W}_{\mathfrak{X}}, e H_{c} e\right)$-bimodule. Similarly, $\mathscr{L}_{c-1}$ has a structure of $\left(\mathscr{W}_{\mathfrak{X}}, e_{\text {det }} H_{c} e_{\text {det }}\right)$-bimodule (Lemma 3.11). These actions are explicitly given by:

$$
\begin{aligned}
& u_{c} e a=\kappa_{0}(a) u_{c} \quad \text { for } a \in \mathbb{C}[\mathfrak{t}]^{W} \subset H_{c} \\
& u_{c} e b=\kappa_{1}(b) u_{c} \quad \text { for } b \in \mathbb{C}\left[\mathfrak{t}^{*}\right]^{W} \subset H_{c} . \\
& u_{c-1} e_{\operatorname{det}} a=\kappa_{0}(a) u_{c-1} \quad \text { for } a \in \mathbb{C}[\mathfrak{t}]^{W} \subset H_{c}, \\
& u_{c-1} e_{\operatorname{det}} b=\kappa_{1}(b) u_{c-1} \quad \text { for } b \in \mathbb{C}\left[\mathfrak{t}^{*}\right]^{W} \subset H_{c} .
\end{aligned}
$$

Since $\mu_{\mathfrak{X}}^{-1}(0)$ is smooth, we have

$$
\mathscr{E} x t_{\mathscr{W}_{\mathfrak{X}}}^{j}\left(\mathscr{L}_{c}, \mathscr{W}_{\mathfrak{X}}\right)=0 \text { for } j \neq \operatorname{codim}_{\mathfrak{X}}\left(\mu_{\mathfrak{X}}^{-1}(0)\right) .
$$

Hence, for any closed subset $S \subset \mu_{\mathfrak{X}}^{-1}(0)$, we have by Lemma 2.1:

$$
\mathscr{H}_{S}^{j}\left(\mathscr{L}_{c}\right)=0 \text { for } j<\operatorname{codim}_{\mu_{\mathfrak{X}}^{-1}(0)} S .
$$

In (3.18) and (3.15), we defined the morphisms:

$$
\varphi: \mathscr{L}_{c} \underset{e H_{c} e}{\otimes} e H_{c} e_{\operatorname{det}} \longrightarrow \mathscr{L}_{c-1} \otimes \operatorname{det}(V)
$$

and

$$
\psi:\left.\left.\left(\mathscr{L}_{c-1} \otimes \operatorname{det}(V)\right) \underset{e_{\mathrm{det}} H_{c} e_{\operatorname{det}}}{\otimes} e_{\operatorname{det}} H_{c} e\right|_{\{q(A, z) \neq 0\}} \longrightarrow \mathscr{L}_{c}\right|_{\{q(A, z) \neq 0\}} .
$$

Proposition 4.2. The morphism $\psi$ extends uniquely to a morphism defined on $\mathfrak{X}$.

Proof. We have

for any $a \in e_{\operatorname{det}} H_{c} e$.

$$
q(A, z) \psi\left(u_{c-1} \otimes a\right)=u_{c} \cdot(\mathfrak{d}(x) a)
$$

Now let us show that

$$
\left(\operatorname{ad}\left(\Delta_{\mathfrak{g}}\right)^{k} q(A, z)\right) \psi\left(u_{c-1} \otimes a\right)=u_{c} \cdot\left(\left(\operatorname{ad}\left(\mathbf{y}^{2}\right)^{k} \mathfrak{d}(x)\right) a\right)
$$

holds on $\{q(A, z) \neq 0\}$ by the induction on $k$.

We have

$$
\begin{aligned}
& \left(\operatorname{ad}\left(\Delta_{\mathfrak{g}}\right)^{k} q(A, z)\right) \psi\left(u_{c-1} \otimes a\right) \\
& \quad=\Delta_{\mathfrak{g}}\left(\operatorname{ad}\left(\Delta_{\mathfrak{g}}\right)^{k-1} q(A, z)\right) \psi\left(u_{c-1} \otimes a\right)-\left(\operatorname{ad}\left(\Delta_{\mathfrak{g}}\right)^{k-1} q(A, z)\right) \Delta_{\mathfrak{g}} \psi\left(u_{c-1} \otimes a\right) .
\end{aligned}
$$

The first term is calculated as

$$
\begin{aligned}
\Delta_{\mathfrak{g}}\left(\operatorname{ad}\left(\Delta_{\mathfrak{g}}\right)^{k-1} q(A, z)\right) \psi\left(u_{c-1} \otimes a\right) & =\Delta_{\mathfrak{g}} u_{c} \cdot\left(\left(\operatorname{ad}\left(\mathbf{y}^{2}\right)^{k-1} \mathfrak{d}(x)\right) a\right) \\
& =u_{c} \mathbf{y}^{2} \cdot\left(\left(\operatorname{ad}\left(\mathbf{y}^{2}\right)^{k-1} \mathfrak{d}(x)\right) a\right) \\
& =u_{c} \cdot\left(\mathbf{y}^{2}\left(\operatorname{ad}\left(\mathbf{y}^{2}\right)^{k-1} \mathfrak{d}(x)\right) a\right)
\end{aligned}
$$

The second term is calculated as

$$
\begin{aligned}
\left(\operatorname{ad}\left(\Delta_{\mathfrak{g}}\right)^{k-1} q(A, z)\right) \Delta_{\mathfrak{g}} \psi\left(u_{c-1} \otimes a\right) & =\left(\operatorname{ad}\left(\Delta_{\mathfrak{g}}\right)^{k-1} q(A, z)\right) \psi\left(\Delta_{\mathfrak{g}} u_{c-1} \otimes a\right) \\
& =\left(\operatorname{ad}\left(\Delta_{\mathfrak{g}}\right)^{k-1} q(A, z)\right) \psi\left(u_{c-1} \mathbf{y}^{2} \otimes a\right) \\
& =\left(\operatorname{ad}\left(\Delta_{\mathfrak{g}}\right)^{k-1} q(A, z)\right) \psi\left(u_{c-1} \otimes \mathbf{y}^{2} a\right) \\
& =u_{c} \cdot\left(\left(\operatorname{ad}\left(\mathbf{y}^{2}\right)^{k-1} \mathfrak{d}(x)\right) \mathbf{y}^{2} a\right)
\end{aligned}
$$


Hence we obtain (4.10). In particular, letting $k$ to be $n(n-1) / 2$, the degree of $\mathfrak{d}(x)$, and using the fact that $\operatorname{ad}\left(\Delta_{\mathfrak{g}}\right)^{n(n-1) / 2} q(A, z)$ is equal to $q\left(\partial_{A}, z\right)$ up to a constant multiple (see e.g. (3.2) and the sentence below), we obtain

$$
q\left(\partial_{A}, z\right) \psi\left(u_{c-1} \otimes a\right)=u_{c} \cdot(\mathfrak{d}(y) a)
$$

Hence $\psi\left(u_{c-1} \otimes a\right)$ extends to a section of $\mathscr{L}_{c}$ outside $q(B, z)=0$.

Thus we have shown that $\psi\left(u_{c-1} \otimes a\right)$ is a section defined outside $\{q(A, z)=0\} \cap$ $\{q(B, z)=0\}$. Since $\{q(A, z)=0\} \cap\{q(B, z)=0\} \cap \mu_{\mathfrak{X}}^{-1}(0)$ is of codimension 2 in $\mu_{\mathfrak{X}}^{-1}(0)$ (Lemma 4.1), it follows that $\psi\left(u_{c-1} \otimes a\right)$ extends to a global section of $\mathscr{L}_{c}$ by $(4.8)$.

Remark 4.3. (i) So, we have obtained a structure of $\left(e+e_{\text {det }}\right) H_{c}\left(e+e_{\text {det }}\right)$-module on $\mathscr{L}_{c} \oplus \mathscr{L}_{c-1} \otimes \operatorname{det}(V)$.

(ii) We have

$$
\begin{aligned}
& \varphi\left(u_{c} \otimes e \mathfrak{d}(x)\right)=q(A, z) u_{c-1}, \\
& \varphi\left(u_{c} \otimes e \mathfrak{d}(y)\right)=q\left(\partial_{A}, z\right) u_{c-1}, \\
& q(A, z) \psi\left(u_{c-1} \otimes a\right)=u_{c} \cdot(\mathfrak{d}(x) a) \quad \text { for } a \in e_{\operatorname{det}} H_{c} e . \\
& q\left(\partial_{A}, z\right) \psi\left(u_{c-1} \otimes a\right)=u_{c} \cdot(\mathfrak{d}(y) a) \quad
\end{aligned}
$$

(iii) The diagrams (3.16) and (3.17) commute on $\mathfrak{X}$.

By Propositions 3.12, 4.2 and Remark 4.3 (iii), we obtain the following proposition (see Proposition 3.8).

Proposition 4.4. Assume Condition (3.12) holds. Then we have isomorphisms of twisted G-equivariant $\mathscr{W}_{\mathfrak{X}}$-modules with twist $\mathrm{ctr}$ :

$$
\varphi: \mathscr{L}_{c} \otimes_{e H_{c} e} e H_{c} e_{\operatorname{det}} \stackrel{\sim}{\longrightarrow} \mathscr{L}_{c-1} \otimes \operatorname{det}(V)
$$

and

$$
\psi:\left(\mathscr{L}_{c-1} \otimes \operatorname{det}(V)\right) \underset{e_{\operatorname{det}} H_{c} e_{\operatorname{det}}}{\otimes} e_{\operatorname{det}} H_{c} e \stackrel{\sim}{\sim} \mathscr{L}_{c} .
$$

\subsubsection{Let us consider}

$$
\mathscr{A}_{c}=\left(p_{*}\left(\mathscr{E} n d_{\mathscr{W}_{\mathfrak{x}}}\left(\mathscr{L}_{c}\right)\right)^{G}\right)^{\mathrm{opp}}
$$

It is a W-algebra on Hilb by Proposition 2.8. Let $\mathscr{A}_{c}(0)$ be the subring of sections of order at most 0 . For $m \in \mathbb{Z}, \mathscr{L}_{c+m} \otimes \operatorname{det}(V)^{\otimes-m}$ belongs to $\operatorname{Mod}_{c \operatorname{tr}}^{G}\left(\mathscr{W}_{\mathfrak{X}}\right)(\operatorname{cf} .(2.4))$. Set

$$
\mathscr{A}_{c, c+m}=\left(p_{*} \mathscr{H}_{o m_{\mathscr{W}_{\mathfrak{x}}}}\left(\mathscr{L}_{c}, \mathscr{L}_{c+m} \otimes \operatorname{det}(V)^{\otimes-m}\right)\right)^{G} .
$$

Then $\mathscr{A}_{c, c+m}$ is an $\left(\mathscr{A}_{c}, \mathscr{A}_{c+m}\right)$-bimodule. Let $\mathscr{A}_{c, c+m}(0)=\left(p_{*} \mathscr{H}_{o m_{W_{x}(0)}}\left(\mathscr{L}_{c}(0), \mathscr{L}_{c+m}(0) \otimes\right.\right.$ $\left.\left.\operatorname{det}(V)^{\otimes-m}\right)\right)^{G}$. Then $\mathscr{A}_{c, c+m}(0)$ is an $\mathscr{A}_{c}(0)$-lattice of $\mathscr{A}_{c, c+m}$ and $\mathscr{A}_{c, c+m}(0) / \mathscr{A}_{c, c+m}(-1) \simeq$ $L^{\otimes-m}$, the associated line bundle on Hilb to $\mathscr{O}_{\mu_{\mathfrak{X}}^{-1}(0)} \otimes \operatorname{det}(V)^{\otimes-m}(\mathrm{cf}$. Proposition 2.8 (iii)).

\subsection{Affinity of $\mathscr{A}_{c}$.}

4.3.1. As an application of Theorem 2.9, we obtain the following vanishing theorem.

Theorem 4.5. Assume Condition (3.12) holds for $c+m$ (for all $m \in \mathbb{Z}_{>0}$ ).

(i) For any good $\mathscr{A}_{c}$-module $\mathscr{M},{\underset{K}{K}}_{\lim } H^{i}(K, \mathscr{M})=0$ for $i>0$. Here $K$ ranges over compact subsets of Hilb. 
(ii) Any good $\mathscr{A}_{c}$-module $\mathscr{M}$ is generated by global sections on any compact subset of Hilb.

Proof. By Proposition 4.4, for any $m>0, \mathscr{L}_{c+m}$ is a direct summand of a direct sum of copies of $\mathscr{L}_{c+m-1} \otimes \operatorname{det}(V)$ and $\mathscr{L}_{c+m-1} \otimes \operatorname{det}(V)$ is a direct summand of a direct sum of copies of $\mathscr{L}_{c+m}$ in the category $\operatorname{Mod}_{(c+m) \operatorname{tr}}^{G}\left(\mathscr{W}_{\mathfrak{X}}\right)$. Hence $\mathscr{L}_{c+m} \otimes \operatorname{det}(V)^{\otimes-m}$ is a direct summand of a direct sum of copies of $\mathscr{L}_{c}$ and $\mathscr{L}_{c}$ is a direct summand of a direct sum of copies of $\mathscr{L}_{c+m} \otimes \operatorname{det}(V)^{\otimes-m}$ in the category $\operatorname{Mod}_{c \text { tr }}^{G}\left(\mathscr{W}_{\mathfrak{X}}\right)$ for any $m>0$. It follows that $\mathscr{A}_{c, c+m}$ is a direct summand of a direct sum of copies of $\mathscr{A}_{c}$ and $\mathscr{A}_{c}$ is a direct summand of a direct sum of copies of $\mathscr{A}_{c, c+m}$ for any $m>0$. Moreover $\mathscr{A}_{c, c+m}$ is a good $\mathscr{A}_{c}$-module whose symbol is $L^{\otimes-m}$.

Theorem 2.9 now gives the conclusion.

4.3.2. Let us give an F-action on $\mathscr{W}_{\mathfrak{X}}$ by $\mathcal{F}_{t}\left(A_{i j}\right)=t A_{i j}, \mathcal{F}_{t}\left(\partial_{A_{i j}}\right)=t^{-1} \partial_{A_{i j}}, \mathcal{F}_{t}\left(z_{i}\right)=t z_{i}$, $\mathcal{F}_{t}\left(\partial_{z_{i}}\right)=t^{-1} \partial_{z_{i}}$ and $\mathcal{F}_{t}(\hbar)=t^{2} \hbar$ for $t \in \mathbb{G}_{\mathrm{m}}=\mathbb{C}^{\times}$. Since $B_{i j}=\sigma_{0}\left(\hbar \partial_{A_{j i}}\right)$ and $\zeta_{i}=$ $\sigma_{0}\left(\hbar \partial_{z_{i}}\right)$, the corresponding action of $\mathbb{G}_{\mathrm{m}}$ on $\mathfrak{X}$ is $T_{t}((A, B, z, \zeta))=(t A, t B, t z, t \zeta)$. Its induced $\mathbb{G}_{\mathrm{m}}$-action on Hilb coincides with the action induced by the scalar $\mathbb{G}_{\mathrm{m}}$-action on $\mathbb{C}^{2}$. We define the F-action on $\mathscr{L}_{c}$ by $\mathcal{F}_{t}\left(u_{c}\right)=u_{c}$.

Note that

$$
\begin{aligned}
\operatorname{End}_{\operatorname{Mod}_{F}\left(\mathscr{W}_{\mathfrak{X}}\left[\hbar^{1 / 2}\right]\right)}\left(\mathscr{W}_{\mathfrak{X}}\left[\hbar^{1 / 2}\right]\right) & \simeq \operatorname{End}_{\operatorname{Mod}_{F}\left(\mathscr{W}_{T^{*}(\mathfrak{g} \times V)}\left[\hbar^{1 / 2}\right]\right)}\left(\mathscr{W}_{T^{*}(\mathfrak{g} \times V)}\left[\hbar^{1 / 2}\right]\right) \\
& \simeq \mathbb{C}\left[\hbar^{-1 / 2} A_{i j}, \hbar^{1 / 2} \partial_{A_{i j}}, \hbar^{-1 / 2} z_{i}, \hbar^{1 / 2} \partial_{z_{i}}\right] \simeq \mathscr{D}(\mathfrak{g} \times V) .
\end{aligned}
$$

The F-action on $\mathscr{W}_{\mathfrak{X}}$ is compatible with the $G$-action on $\mathscr{W}$, and hence $\mathscr{A}_{c}$ is also a W-algebra on Hilb with F-action (cf. Proposition 2.8 (iv)). We define the F-action on $\mathscr{L}_{c-1} \otimes \operatorname{det}(V)$ by $\mathcal{F}_{t}\left(u_{c-1} \otimes l\right)=t^{-n} u_{c-1} \otimes l$. Hence $\mathscr{A}_{c, c-1}$ has a structure of $\mathscr{A}_{c}$-module with F-action.

4.3.3. The $\left(\left(e+e_{\mathrm{det}}\right) H_{c}\left(e+e_{\mathrm{det}}\right)\right)^{\mathrm{opp}}$-module structure on $\mathscr{L}_{c} \oplus\left(\mathscr{L}_{c-1} \otimes \operatorname{det}(V)\right)$ gives a ring homomorphism

$$
\left(e+e_{\mathrm{det}}\right) H_{c}\left(e+e_{\mathrm{det}}\right) \stackrel{\alpha}{\rightarrow} \operatorname{End}_{\mathscr{A}_{c}}\left(\mathscr{A}_{c} \oplus \mathscr{A}_{c, c-1}\right)^{\mathrm{opp}} .
$$

Since it is not compatible with the F-action, we shall modify $\alpha$.

Set

$$
\widetilde{\mathscr{A}_{c}}=\mathscr{A}_{c}\left[\hbar^{1 / 2}\right] \quad \text { and } \quad \widetilde{\mathscr{A}}_{c, c-1}=\mathscr{A}_{c, c-1}\left[\hbar^{1 / 2}\right]
$$

Let $H_{c} \stackrel{\beta}{\rightarrow} \mathbf{k}\left[\hbar^{1 / 2}\right] \otimes_{\mathbb{C}} H_{c}$ be the ring homomorphism given by $x_{i} \mapsto \hbar^{-1 / 2} \otimes x_{i}, y_{i} \mapsto \hbar^{1 / 2} \otimes y_{i}$, $w \mapsto 1 \otimes w(w \in W)$.

Lemma 4.6. The composition

$$
\begin{aligned}
& \Phi:\left(e+e_{\mathrm{det}}\right) H_{c}\left(e+e_{\mathrm{det}}\right) \stackrel{\beta}{\rightarrow} \mathbf{k}\left[\hbar^{1 / 2}\right] \otimes_{\mathbb{C}}\left(e+e_{\mathrm{det}}\right) H_{c}\left(e+e_{\mathrm{det}}\right) \stackrel{\alpha}{\rightarrow} \operatorname{End}_{\widetilde{\mathscr{A}}_{c}}\left(\widetilde{\mathscr{A}}_{c} \oplus \widetilde{\mathscr{A}}_{c, c-1}\right)^{\mathrm{opp}} \\
& \text { sends }\left(e+e_{\mathrm{det}}\right) H_{c}\left(e+e_{\mathrm{det}}\right) \text { to } \operatorname{End}_{\mathrm{Mod}_{F}\left(\widetilde{\mathscr{A}_{c}}\right)}\left(\widetilde{\mathscr{A}_{c}} \oplus \widetilde{\mathscr{A}}_{c, c-1}\right)^{\mathrm{opp}} .
\end{aligned}
$$

Proof. First let us show that $\Phi$ sends $e H_{c} e$ to $\operatorname{End}_{\operatorname{Mod}_{F}\left(\widetilde{\mathscr{A}_{c}}\right)}\left(\widetilde{\mathscr{A}_{c}}\right)^{\text {opp }}$. For a homogeneous element $a \in \mathbb{C}[\mathfrak{t}]^{W}$ of degree $k, \Phi(a e)\left(u_{c}\right)=\hbar^{-k / 2} \tilde{a}(A) u_{c}$, where $\tilde{a}(A)$ is the element of $\mathbb{C}[\mathfrak{g}]^{G}$ such that $\left.\tilde{a}\right|_{\mathfrak{t}}=a$. Since $\tilde{a}(A)$ is also homogeneous of degree $k, \hbar^{-k / 2} \tilde{a}(A)$ is $\mathcal{F}$-invariant, and $\Phi(a e)$ belongs to $\operatorname{Mod}_{F}\left(\widetilde{\mathscr{A}_{c}}\right)$. On the other hand, we have $\Phi\left(\mathbf{y}^{2} e\right)\left(u_{c}\right)=\hbar \Delta_{\mathfrak{g}} u_{c}$ and 
$\hbar \Delta_{\mathfrak{g}}$ is $\mathcal{F}$-invariant. Hence $\Phi\left(\mathbf{y}^{2} e\right)$ belongs to $\operatorname{Mod}_{F}\left(\widetilde{\mathscr{A}_{c}}\right)$. Since $e H_{c} e$ is generated by $\mathbb{C}[\mathfrak{t}]^{W} e$ and $\mathbf{y}^{2} e$, we have $\Phi\left(e H_{c} e\right) \subset \operatorname{End}_{\operatorname{Mod}_{F}\left(\widetilde{\mathscr{A}_{c}}\right)}\left(\widetilde{\mathscr{A}_{c}}\right)$.

Similarly, we have $\Phi\left(e_{\operatorname{det}} H_{c} e_{\mathrm{det}}\right) \subset \operatorname{End}_{\operatorname{Mod}_{F}\left(\widetilde{\mathscr{A}_{c}}\right)}\left(\widetilde{\mathscr{A}_{c, c-1}}\right)$.

Let us show that $\Phi(e \mathfrak{d}(x)) \in \operatorname{Hom}_{\operatorname{Mod}_{F}\left(\widetilde{\mathscr{A}}_{c}\right)}\left(\widetilde{\mathscr{A}}_{c}, \widetilde{\mathscr{A}}_{c, c-1}\right)$. This follows from $\Phi(e \mathfrak{d}(x))\left(u_{c}\right)=$ $\hbar^{-n(n-1) / 4} q(A, z) u_{c-1} \otimes l, \mathcal{F}_{t}(q(A, z))=t^{n+n(n-1) / 2} q(A, z)$ and $\mathcal{F}_{t}\left(u_{c-1} \otimes l\right)=t^{-n} u_{c-1} \otimes l$.

For $a \in e_{\text {det }} H_{c} e$, let us show that $\Phi(a): \widetilde{\mathscr{A}}_{c, c-1} \rightarrow \widetilde{\mathscr{A}_{c}}$ belongs to $\operatorname{Mod}_{F}\left(\widetilde{\mathscr{A}_{c}}\right)$. Since $\Phi($ aed $(x))$ belongs to $\operatorname{Mod}_{F}\left(\widetilde{\mathscr{A}_{C}}\right)$, and since $\left.\Phi(e \mathfrak{d}(x))\right|_{\{q(A, z) \neq 0\}}$ is an isomorphism in the category $\operatorname{Mod}_{F}\left(\left.\widetilde{\mathscr{A}_{c}}\right|_{\{q(A, z) \neq 0\}}\right)$, it follows that $\left.\Phi(a)\right|_{\{q(A, z) \neq 0\}}$ is in $\operatorname{Mod}_{F}\left(\left.\widetilde{\mathscr{A}}_{c}\right|_{\{q(A, z) \neq 0\}}\right)$. Hence we conclude that $\Phi(a)$ is in $\operatorname{Mod}_{F}\left(\widetilde{\mathscr{A}_{c}}\right)$. Similarly, one shows that $\Phi\left(e H_{c} e_{\text {det }}\right)$ is contained in $\operatorname{Hom}_{\operatorname{Mod}_{F}\left(\widetilde{\mathscr{A}}_{c}\right)}\left(\widetilde{\mathscr{A}}_{c}, \widetilde{\mathscr{A}}_{c, c-1}\right)$.

In particular we obtain a morphism of algebras

$$
e H_{c} e \rightarrow \operatorname{End}_{\operatorname{Mod}_{F}\left(\widetilde{\mathscr{A}_{c}}\right)}\left(\widetilde{\mathscr{A}_{c}}\right)^{\text {opp }} \text {. }
$$

We denote by $\tilde{\varphi}$ and $\tilde{\psi}$ the modified morphisms in $\operatorname{Mod}_{F}\left(\widetilde{\mathscr{A}_{c}}\right)$ given in Lemma 4.6:

$$
\begin{array}{rll}
\tilde{\varphi} & : & \widetilde{\mathscr{L}}_{c} \otimes_{e H_{c} e} e H_{c} e_{\mathrm{det}} \longrightarrow \widetilde{\mathscr{L}}_{c-1} \otimes \operatorname{det}(V), \\
\tilde{\psi}: & \left(\widetilde{\mathscr{L}}_{c-1} \otimes \operatorname{det}(V)\right) \otimes_{e_{\mathrm{det}} H_{c} e_{\mathrm{det}}} e_{\operatorname{det}} H_{c} e \longrightarrow \widetilde{\mathscr{L}}_{c} .
\end{array}
$$

We define the order filtration $F\left(e H_{c} e\right)$ on $e H_{c} e$ by assigning order $1 / 2$ to $x_{i}$ and $y_{i}$. Then the morphism $e H_{c} e \rightarrow \operatorname{End}_{\operatorname{Mod}_{F}\left(\widetilde{\mathscr{A}_{c}}\right)}\left(\widetilde{\mathscr{A}_{c}}\right)^{\text {opp }}$ is compatible with the order filtrations, and the symbol map $\mathbb{C}\left[\mathfrak{t} \times \mathfrak{t}^{*}\right]^{W} \simeq \operatorname{Gr}^{F}\left(e H_{c} e\right) \rightarrow \operatorname{Gr}^{F} \operatorname{End}_{\operatorname{Mod}_{F}\left(\tilde{\mathscr{A}}_{c}\right)}\left(\widetilde{\mathscr{A}_{c}}\right) \subset \Gamma\left(\right.$ Hilb, $\left.\mathscr{O}_{\text {Hilb }}\right)\left[\hbar^{ \pm 1 / 2}\right]$ coincides with $\mathbb{C}\left[\mathfrak{t} \times \mathfrak{t}^{*}\right]_{k}^{W} \stackrel{\hbar^{-k} i_{s}}{\longrightarrow} \hbar^{-k} \Gamma\left(\right.$ Hilb, $\left.\mathscr{O}_{\text {Hilb }}\right)$ by (4.5). Here $k \in \mathbb{Z} / 2$.

Lemma 4.7. The morphism $e H_{c} e \rightarrow \operatorname{End}_{\operatorname{Mod}_{F}\left(\widetilde{\mathscr{A}_{c}}\right)}\left(\widetilde{\mathscr{A}_{c}}\right)^{\mathrm{opp}}$ is an isomorphism.

Proof. Note that the subspace $\operatorname{Gr}^{F} \operatorname{End}_{\operatorname{Mod}_{F}\left(\widetilde{\mathscr{A}_{c}}\right)}\left(\widetilde{\mathscr{A}_{c}}\right) \subset \Gamma\left(\right.$ Hilb, $\left.\mathscr{O}_{\text {Hilb }}\right)\left[\hbar^{ \pm 1 / 2}\right]$ is contained in $\oplus_{k \in \mathbb{Z} / 2} \Gamma\left(\mathrm{Hilb}, \mathscr{O}_{\mathrm{Hilb}}\right)_{k} \hbar^{-k}$ where $\Gamma\left(\mathrm{Hilb}, \mathscr{O}_{\mathrm{Hilb}}\right)_{k}$ is the homogeneous part of weight $2 k$ with respect to the $\mathbb{G}_{\mathrm{m}}$-action. Hence we have a chain of morphisms

$$
\begin{aligned}
\mathbb{C}\left[\mathfrak{t} \times \mathfrak{t}^{*}\right]^{W} & \stackrel{\sim}{\sim} \operatorname{Gr}^{F}\left(e H_{c} e\right) \\
& \rightarrow \operatorname{Gr}^{F}\left(\operatorname{End}_{\operatorname{Mod}_{F}\left(\widetilde{\mathscr{A}_{c}}\right)}\left(\widetilde{\mathscr{A}_{c}}\right)^{\text {opp }}\right) \hookrightarrow \oplus_{k \in \mathbb{Z} / 2} \Gamma\left(\mathrm{Hilb}, \mathscr{O}_{\mathrm{Hilb}}\right)_{k} \hbar^{-k} \stackrel{\sim}{\longrightarrow}\left[\mathfrak{t} \times \mathfrak{t}^{*}\right]^{W}
\end{aligned}
$$

Since the composition is the identity, the $\operatorname{map} \operatorname{Gr}^{F}\left(e H_{c} e\right) \rightarrow \operatorname{Gr}^{F}\left(\operatorname{End}_{\operatorname{Mod}_{F}\left(\widetilde{\mathscr{A}_{c}}\right)}\left(\widetilde{\mathscr{A}_{c}}\right)^{\text {opp }}\right)$ is bijective. Hence the morphism $e H_{c} e \rightarrow \operatorname{End}_{\operatorname{Mod}_{F}\left(\widetilde{\mathscr{A}_{c}}\right)}\left(\widetilde{\mathscr{A}_{c}}\right)^{\text {opp }}$ is an isomorphism. Note that $\bigcap_{k} F_{k}\left(\operatorname{End}_{\operatorname{Mod}_{F}\left(\widetilde{\mathscr{A}_{c}}\right)}\left(\widetilde{\mathscr{A}_{c}}\right)\right)=0$.

Remark 4.8. A similar argument shows that there is an isomorphism

(See $\S 4.4$.

$$
e H_{c} e_{\mathrm{det}} \stackrel{\sim}{\longrightarrow} \operatorname{Hom}_{\operatorname{Mod}_{F}\left(\widetilde{\mathscr{A}}_{c}\right)}\left(\widetilde{\mathscr{A}_{c}}, \widetilde{\mathscr{A}}_{c, c-1}\right) \text {. }
$$

Let $\mathrm{o} \in\left(\mathfrak{t} \times \mathfrak{t}^{*}\right) / W$ be the image of the origin of $\mathfrak{t} \times \mathfrak{t}^{*}$. Then the Hilbert-Chow morphism $\pi:$ Hilb $\rightarrow\left(\mathfrak{t} \times \mathfrak{t}^{*}\right) / W$ is $\mathbb{C}^{\times}$-equivariant, and every point of $\left(\mathfrak{t} \times \mathfrak{t}^{*}\right) / W$ shrinks to o.

Now the following theorem is a consequence of Theorem 2.10. 
Theorem 4.9. Assume Condition (3.12) holds for $c+m$, for all $m \in \mathbb{Z}_{>0}$ (this will be the case if $\left.c \notin \frac{1}{n !} \mathbb{Z}_{<0}\right)$. We have quasi-inverse equivalences of categories between $\operatorname{Mod}_{F}^{\text {good }}\left(\widetilde{\mathscr{A}_{c}}\right)$ and $\operatorname{Mod}_{\mathrm{coh}}\left(e H_{c} e\right)$

$$
\begin{aligned}
& \operatorname{Mod}_{F}^{\text {good }}\left(\widetilde{\mathscr{A}_{c}}\right) \stackrel{\sim}{\longleftrightarrow} \operatorname{Mod}_{\mathrm{coh}}\left(e H_{c} e\right) \\
& \mathscr{M} \mapsto \operatorname{Hom}_{\operatorname{Mod}_{F}^{\text {good }}\left(\widetilde{\mathscr{A}_{c}}\right)}\left(\widetilde{\mathscr{A}_{c}}, \mathscr{M}\right) \\
& \widetilde{\mathscr{A}_{c}} \otimes_{e H_{c} e} M \leftarrow M .
\end{aligned}
$$

Under this equivalence, $\widetilde{\mathscr{A}_{c}}$ and $\widetilde{\mathscr{A}}_{c, c-1}$ correspond to $e H_{c} e$ and $e H_{c} e_{\mathrm{det}}$, respectively.

Theorem 4.10. Assume Condition (3.12) holds for $c+m$ (for all $\left.m \in \mathbb{Z}_{>0}\right)$. Assume also Condition (3.11) holds (these assumptions will be satisfied if $c \notin \frac{1}{n !} \mathbb{Z}_{<0}$ ). Let $\mathcal{B}_{c}=\mathscr{E}_{n} d_{\widetilde{A}_{c}}\left(\widetilde{\mathscr{A}_{c}} \otimes_{e H_{c} e} e H_{c}\right)^{\text {opp }}$. We have quasi-inverse equivalences of categories between $\operatorname{Mod}_{F}^{\text {good }}\left(\mathcal{B}_{c}\right)$ and $\operatorname{Mod}_{\text {coh }}\left(H_{c}\right)$

$$
\begin{aligned}
& \operatorname{Mod}_{F}^{\text {good }}\left(\mathcal{B}_{c}\right) \stackrel{\sim}{\sim} \operatorname{Mod}_{\mathrm{coh}}\left(H_{c}\right) \\
& \mathscr{M} \mapsto \operatorname{Hom}_{\operatorname{Mod}_{F}^{\text {good }}\left(\mathcal{B}_{c}\right)}\left(\mathcal{B}_{c}, \mathscr{M}\right) \\
& \mathcal{B}_{c} \otimes_{H_{c}} M \quad \longleftrightarrow \quad M .
\end{aligned}
$$

Remark 4.11. It would be very interesting to have a more direct construction of $\widetilde{\mathscr{A}_{c}} \otimes_{e H_{c} e}$ $e H_{c}$.

4.4. W-algebras as fractions of $e H_{c} e$. We explain how sections of $\widetilde{\mathscr{A}_{c}}$ over open subsets of Hilb can be obtained by inverting elements in the Cherednik algebra.

Let $\left\{F_{j}\left(H_{c}\right)\right\}_{j \in \mathbb{Z} / 2}$ be the filtration of $H_{c}$ consisting of elements of order $\leqslant j$, where we give order $1 / 2$ to $x_{i}, y_{i}$ and order 0 to $w \in W$. Then we have a canonical isomorphism $\sigma: \operatorname{Gr}^{F}\left(H_{c}\right) \stackrel{\sim}{\longrightarrow}\left[\mathfrak{t} \times \mathfrak{t}^{*}\right] \rtimes W$. We have induced filtrations on $e H_{c} e$ and $e H_{c} e_{\mathrm{det}}$, and $\sigma$ induces isomorphisms

$$
\begin{aligned}
& \mathrm{Gr}^{F}\left(e H_{c} e\right) \stackrel{\sim}{\longrightarrow}\left[\mathfrak{t} \times \mathfrak{t}^{*}\right]^{W}, \\
& \operatorname{Gr}^{F}\left(e H_{c} e_{\text {det }}\right) \stackrel{\sim}{\longrightarrow}\left[\mathfrak{t} \times \mathfrak{t}^{*}\right]^{W, \operatorname{det}} .
\end{aligned}
$$

Composing with the morphism $\mathbb{C}\left[\mathfrak{t} \times \mathfrak{t}^{*}\right] \rightarrow \mathbb{C}\left[\mathfrak{t} \times \mathfrak{t}^{*}\right]\left[\hbar^{-1 / 2}\right]$ given by $a(x, y) \mapsto a\left(\hbar^{-1 / 2} x, \hbar^{-1 / 2} y\right)$, we obtain homomorphisms

$$
\begin{aligned}
\operatorname{Gr}^{F}\left(e H_{c} e\right) & \longrightarrow \mathbb{C}\left[\mathfrak{t} \times \mathfrak{t}^{*}\right]^{W}\left[\hbar^{-1 / 2}\right], \\
\operatorname{Gr}^{F}\left(e H_{c} e_{\text {det }}\right) & \longrightarrow \mathbb{C}\left[\mathfrak{t} \times \mathfrak{t}^{*}\right]^{W, \operatorname{det}}\left[\hbar^{-1 / 2}\right] .
\end{aligned}
$$

We shall set $\widetilde{\mathscr{W}_{\mathfrak{X}}}=\mathscr{W}_{\mathfrak{x}}\left[\hbar^{1 / 2}\right]$ and $\widetilde{\mathscr{W}_{\mathfrak{X}}}(0)=\mathscr{W}_{\mathfrak{X}}(0)+\hbar^{1 / 2} \mathscr{W}_{\mathfrak{X}}(0)$. We set $\widetilde{\mathscr{L}_{c}}=\widetilde{\mathscr{W}_{\mathfrak{X}}} \otimes_{\mathscr{W}_{\mathfrak{x}}} \mathscr{L}_{c}$. Then $\widetilde{\mathscr{L}_{c}} \oplus \widetilde{\mathscr{L}_{c-1}} \otimes \operatorname{det}(V)$ has a structure of $\left(\widetilde{\mathscr{W}},\left(e+e_{\operatorname{det}}\right) H_{c}\left(e+e_{\operatorname{det}}\right)\right)$-bimodule. The action of $e H_{c} e_{\text {det }}$ is given by $\tilde{\varphi}: \widetilde{\mathscr{L}}_{c} \otimes_{e H_{c} e} e H_{c} e_{\operatorname{det}} \rightarrow \widetilde{\mathscr{L}}_{c-1} \otimes \operatorname{det}(V)$. On the other hand, we have canonical isomorphisms $\operatorname{Gr}^{F}\left(\widetilde{\mathscr{L}_{c}}\right) \simeq \operatorname{Gr}^{F}\left(\widetilde{\mathscr{L}}_{c-1}\right) \stackrel{\sim}{\longrightarrow} \mathscr{O}_{\mu_{\mathfrak{X}}^{-1}(0)}\left[\hbar^{ \pm 1 / 2}\right]$. Here $F\left(\widetilde{\mathscr{L}}_{c}\right)$ $\left(\operatorname{resp} . F\left(\widetilde{\mathscr{L}}_{c-1}\right)\right)$ is the order filtration given by $F_{k}\left(\widetilde{\mathscr{L}}_{c}\right)=\hbar^{-k \widetilde{\mathscr{W}_{\mathfrak{X}}}}(0) u_{c}\left(\operatorname{resp} . F_{k}\left(\widetilde{\mathscr{L}}_{c-1}\right)=\right.$ $\left.\hbar^{-k \widetilde{W}_{\mathfrak{X}}}(0) u_{c-1}\right)$ for $k \in \mathbb{Z} / 2$. 
We have a commutative diagram:

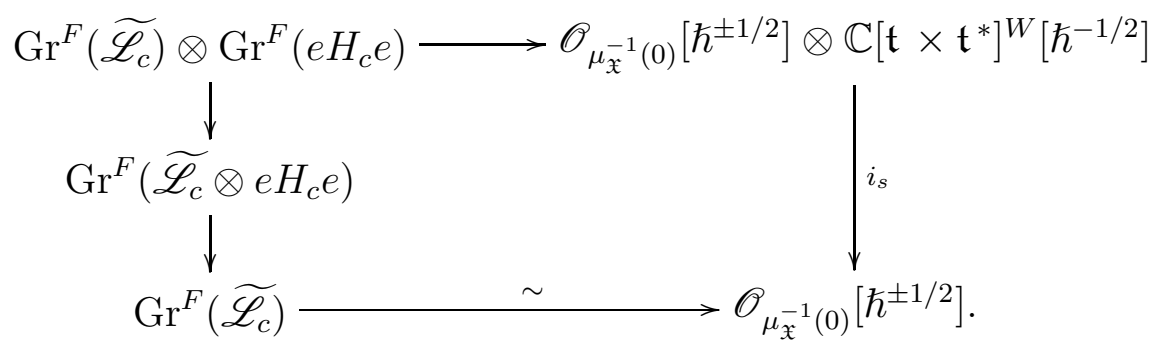

The morphism $\tilde{\varphi}$ is order-preserving and we obtain a commutative diagram

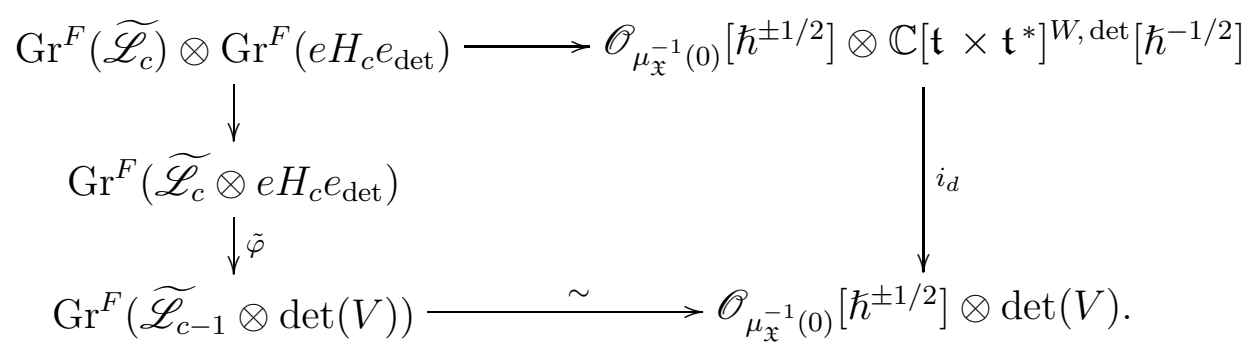

Hence, for any $a \in e H_{c} e_{\mathrm{det}}$, the morphism $a: \widetilde{\mathscr{L}_{c}} \rightarrow \widetilde{\mathscr{L}}_{c-1} \otimes \operatorname{det}(V)$ is an isomorphism on $\left\{i_{d}(\sigma(a)) \neq 0\right\}$. Then, for $b \in e H_{c} e_{\operatorname{det}}$, we can define

$$
b a^{-1} \in \operatorname{End}_{\operatorname{Mod}_{F, c \operatorname{tr}}^{G}\left(\left.\widetilde{\mathscr{W}}_{X}\right|_{\{i d}(\sigma(a)) \neq 0\right\}}\left(\left.\widetilde{\mathscr{L}}_{c}\right|_{\left\{i_{d}(\sigma(a)) \neq 0\right\}}\right)^{\text {opp }}
$$

as the composition

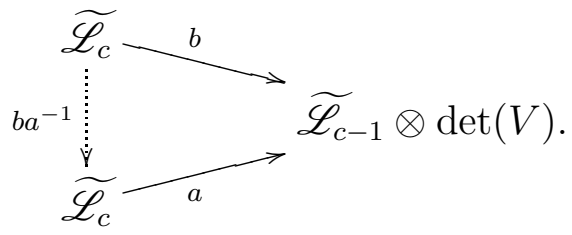

Thus we obtain $b a^{-1}$ as an F-invariant section of $\widetilde{\mathscr{A}}_{c}$ defined on $\left\{i_{d}(\sigma(a)) \neq 0\right\}$. Note that $b a^{-1}=b c(a c)^{-1}$ for a non-zero element $c \in e_{\operatorname{det}} H_{c} e$. Note also that the image of $a c \in e H_{c} e$ in $\Gamma\left(\right.$ Hilb; $\left.\widetilde{\mathscr{A}_{c}}\right)$ is invertible only on $\left\{i_{d}(\sigma(a)) \neq 0\right\} \cap\left\{i_{d}(\sigma(c)) \neq 0\right\} \cap$ (Hilb $\left.\backslash E\right)$.

Remark 4.12. The morphism $\tilde{\psi}:\left(\widetilde{\mathscr{L}}_{c-1} \otimes \operatorname{det}(V)\right) \otimes_{e_{\operatorname{det}} H_{c} e_{\operatorname{det}}} e_{\operatorname{det}} H_{c} e \rightarrow \widetilde{\mathscr{L}}_{c}$ is also orderpreserving, and it induces a commutative diagram

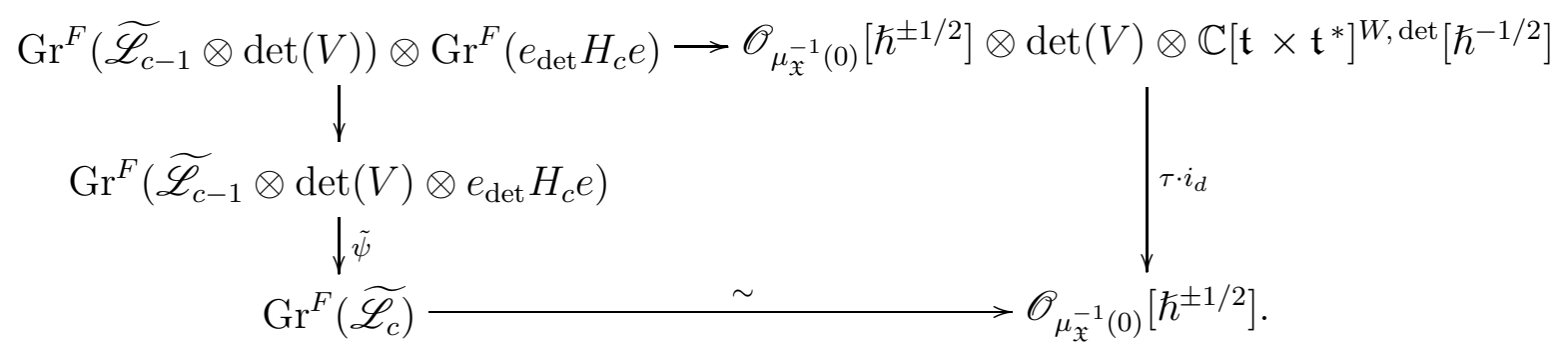

Hence, for any $b \in e_{\operatorname{det}} H_{c} e$, the morphism $b: \widetilde{\mathscr{L}}_{c-1} \otimes \operatorname{det}(V) \rightarrow \widetilde{\mathscr{L}}_{c}$ is never an isomorphism on the exceptional divisor $E$. 
4.5. Rank 2 case. Let us consider the case $n=2$. Let $x_{0}=x_{1}+x_{2}, x=x_{1}-x_{2}$, $y_{0}=\left(y_{1}+y_{2}\right) / 2$ and $y=\left(y_{1}-y_{2}\right) / 2 \in H_{c}$. Then $\left[y_{0}, x_{0}\right]=1,[y, x]=1-2 c s$ where $s=s_{12}$. Since $y, x$ and $s$ commute with $\mathbb{C}\left[x_{0}, y_{0}\right]$, we have an isomorphism of algebras $\mathbb{C}\left[x_{0}, y_{0}\right] \otimes H_{c}^{\prime} \stackrel{\sim}{\longrightarrow} H_{c}$, where $H_{c}^{\prime}$ is the subalgebra of $H_{c}$ generated by $x, y$ and $s$.

We have

$$
\begin{aligned}
& e H_{c} e_{\mathrm{det}} H_{c} e=e H_{c} e \Longleftrightarrow H_{c} e_{\mathrm{det}} H_{c}=H_{c} \Longleftrightarrow c \neq 1 / 2, \\
& e_{\mathrm{det}} H_{c} e H_{c} e_{\mathrm{det}}=e_{\mathrm{det}} H_{c} e_{\operatorname{det}} \Longleftrightarrow H_{c} e H_{c}=H_{c} \Longleftrightarrow c \neq-1 / 2 .
\end{aligned}
$$

Indeed, the first equivalences follow from the fact that $y e_{\operatorname{det}} x-x e_{\operatorname{det}} y=e[y, x]=(1-2 c) e$ and when $c=1 / 2$, there is a one-dimensional representation with $x, y \mapsto 0, s \mapsto 1$. The second follows from the first by the isomorphism $H_{c} \simeq H_{-c}$ given by $s \mapsto-s$. It follows that Condition (3.12) is satisfied for all $c+n\left(n \in \mathbb{Z}_{>0}\right)$ if and only if $c \neq-1 / 2,-3 / 2, \ldots$

Note that $x, y \in \mathbb{C}\left[\mathfrak{t} \times \mathfrak{t}^{*}\right]^{W, \text { det }}$ and Hilb $=\left\{i_{d}(x) \neq 0\right\} \cup\left\{i_{d}(y) \neq 0\right\}$, because $\mu_{\mathfrak{X}}^{-1}(0) \cap$ $\{q(A, z)=q(B, z)=0\} \subset\{(A, B, z, 0) \in \mathfrak{X} ; A z, B z \in \mathbb{C} z\}=\emptyset$. Quantized symplectic coordinates of $\widetilde{\mathscr{A}_{c}}$ are given by

$$
\left((e y)(e x)^{-1}, \hbar^{1 / 2} e x_{0} ;-\hbar e x^{2} / 2, \hbar^{1 / 2} e y_{0}\right) \quad \text { on }\left\{i_{d}(x) \neq 0\right\}
$$

and

$$
\left((e x)(e y)^{-1}, \hbar^{1 / 2} e x_{0} ; \hbar e y^{2} / 2, \hbar^{1 / 2} e y_{0}\right) \quad \text { on }\left\{i_{d}(y) \neq 0\right\} .
$$

Indeed, we have $\left[-e x^{2} / 2,(e y)(e x)^{-1}\right]=e$, because

$$
\begin{gathered}
(e y)(e x)^{-1}\left(e x^{2}\right)=(e y)(e x)^{-1}(e x)\left(e_{\operatorname{det}} x\right)=e y x \quad \text { and } \\
\left(e x^{2}\right)(e y)(e x)^{-1}=\left(e x^{2} y\right)(e x)^{-1}=\left(e y x^{2}-2 e x\right)(e x)^{-1}=(e y x)(e x)(e x)^{-1}-2 e=e y x-2 e .
\end{gathered}
$$

Note that this provides an isomorphism Hilb $\sim T^{*}\left(\mathbb{P}^{1} \times \mathbb{C}\right)$. The projection Hilb $\rightarrow$ $\mathbb{P}^{1}$ is given by $\left[i_{d}(x): i_{d}(y)\right]$ with the notation of homogeneous coordinates. By the isomorphism above, we have $E \simeq T_{\mathbb{P}^{1}}^{*} \mathbb{P}^{1} \times T^{*} \mathbb{C}$.

Note that $(x e)^{-1}(y e)$ is invertible only on $\left\{i_{s}\left(x^{2}\right) \neq 0\right\}=\left\{i_{d}(x) \neq 0\right\} \backslash E$ for $c \neq-1 / 2$, because $e x y x=e x(x y+1-2 c s)=e x^{2} y+(1+2 c) e x$ and $(x e)^{-1}(y e)=\left(x^{2} e\right)^{-1}(x y e)=$ $\left(e x^{2}\right)^{-1}(e x y x)(e x)^{-1}=(e y)(e x)^{-1}+(1+2 c)\left(e x^{2}\right)^{-1}$.

Set $\left(a, \partial_{a}\right)=\left((e y)(e x)^{-1},-e x^{2} / 2\right)$ and $\left(b, \partial_{b}\right)=\left(\left((e x)(e y)^{-1}, e y^{2} / 2\right)\right.$ and $\lambda=c-1 / 2$. Then we have

$$
b=a^{-1} \quad \text { and } \quad \partial_{b}=-a\left(a \partial_{a}-\lambda\right)
$$

Indeed, we have

$$
\begin{aligned}
-a\left(a \partial_{a}-\lambda\right) & =(e y)(e x)^{-1}\left((e y)(e x)^{-1}\left(e x^{2}\right) / 2+c-1 / 2\right) \\
& =(1 / 2)(e y)(e x)^{-1}(e y x+2 c-1)=(1 / 2)(e y)(e x)^{-1}(e x y)=e y^{2} / 2 .
\end{aligned}
$$

Recall that $o \in\left(\mathfrak{t} \times \mathfrak{t}^{*}\right) / W$ is the image of the origin of $\mathfrak{t} \times \mathfrak{t}^{*}$. The inverse image $\pi^{-1}(\mathrm{o})$ by the Hilbert-Chow morphism $\pi$ is $T_{\mathbb{P}^{1}}^{*} \mathbb{P}^{1} \times\{0\} \subset T^{*} \mathbb{P}^{1} \times T^{*} \mathbb{C}$. We identify it with $\mathbb{P}^{1}$. Then, (4.15) gives an isomorphism

$$
\left.\mathscr{E} n d_{F}\left(\widetilde{\mathscr{A}_{C}}\right)\right|_{\pi^{-1}(\mathrm{o})} \stackrel{\sim}{\sim} \mathscr{D}_{\mathbb{P}^{1}, \lambda} \otimes \mathbb{C}\left[x_{0}, y_{0}\right]
$$

with $\lambda=c-1 / 2$. Here, $\mathscr{D}_{\mathbb{P}^{1}, \lambda}$ is the twisted ring of differential operators (e.g. see $\left.[17, \S 2]\right)$. If $\lambda$ is an integer, then $\mathscr{D}_{\mathbb{P}^{1}, \lambda} \simeq \mathscr{O}_{\mathbb{P}^{1}}(\lambda) \otimes \mathscr{D}_{\mathbb{P}^{1}} \otimes \mathscr{O}_{\mathbb{P}^{1}}(-\lambda)$. Hence, we have a ring isomorphism $e H_{c}^{\prime} e \simeq \Gamma\left(\mathbb{P}^{1} ; \mathscr{D}_{\mathbb{P}^{1}, \lambda}\right)$ and an equivalence $\operatorname{Mod}_{F}^{\text {good }}\left(\widetilde{\mathscr{A}_{c}}\right) \simeq \operatorname{Mod}_{\text {good }}\left(\mathscr{D}_{\mathbb{P}^{1}, \lambda} \otimes \mathbb{C}\left[x_{0}, y_{0}\right]\right)$. It is 
well-known (cf. e.g. $[17, \S 7])$ that $\operatorname{Mod}_{\text {good }}\left(\mathscr{D}_{\mathbb{P}^{1}, \lambda}\right)$ is equivalent to $\operatorname{Mod}_{\text {coh }}\left(\Gamma\left(\mathbb{P}^{1} ; \mathscr{D}_{\mathbb{P}^{1}, \lambda}\right)\right)$ if and only if $\lambda \neq-1,-2, \ldots$ (i.e. $c \neq-1 / 2,-3 / 2, \ldots$ ).

\section{REFERENCES}

[1] Beilinson, Alexandre and Bernstein, Joseph, Localisation de g-modules, C. R. Acad. Sci. Paris Sr. I Math. 292 (1981), no. 1, 15-18.

[2] Berest, Yuri, Etingof, Pavel and Ginzburg, Victor, Cherednik algebras and differential operators on quasi-invariants, Duke Math. J. 118 (2003), 279-337.

[3] Bezrukavnikov, Roman and Etingof, Pavel, Parabolic induction and restriction functors for rational Cherednik algebras, preprint, 2007.

[4] Bezrukavnikov, Roman, Finkelberg, Michael and Ginzburg, Victor, Cherednik algebras and Hilbert schemes in characteristic p, with an appendix by Pavel Etingof, Represent. Theory 10 (2006), 254298 (electronic).

[5] Bourbaki, Nicolas, Lie groups and Lie algebras. Chapters 4, 5 and 6,Masson, Paris, 1981. 290 pp.

[6] Etingof, Pavel and Ginzburg, Victor, Symplectic reflection algebras, Calogero-Moser space, and deformed Harish-Chandra homomorphism, Invent. Math. 147 (2002), no. 2, 243-348.

[7] Gan, Wee Liang and Ginzburg, Victor, Almost-commuting variety, $\mathscr{D}$-modules, and Cherednik algebras, IMRP Int. Math. Res. Pap. 2006, 26439, 1-54.

[8] Gordon, Iain and Stafford, J. T., Rational Cherednik algebras and Hilbert schemes, Adv. Math. 198 (2005), no. 1, 222-274.

[9] _ Rational Cherednik algebras and Hilbert schemes. II. Representations and sheaves, Duke Math. J. 132 (2006), no. 1, 73-135.

[10] Guillemin, Victor and Sternberg, Shlomo, Symplectic techniques in physics, Cambridge University Press, 1984.

[11] Haiman, Mark, Vanishing theorems and character formulas for the Hilbert scheme of points in the plane, Invent. Math. 149 (2002), no. 2, 371-407.

[12] Hilbert schemes, polygraphs and the Macdonald positivity conjecture, J. Amer. Math. Soc. 14 (2001), no. 4, 941-1006 (electronic).

[13] Heckmann, Gerrit J., A remark on the Dunkl differential-difference operators, Harmonic analysis on reductive groups (Brunswick, ME, 1989), 181-191, Progr. Math., 101, Birkhäuser, 1991.

[14] Hotta, Ryoshi and Kashiwara, Masaki, The invariant holonomic system on a semisimple Lie algebra, Invent. Math. 75 (1984), no. 2, 327-358.

[15] Kaledin, Dmitry, Geometry and topology of symplectic resolutions, preprint math.AG/0608143.

[16] Kashiwara, Masaki, Quantization of contact manifolds, Publ. Res. Inst. Math. Sci. 32 (1996), no. 1, $1-7$.

[17] _ Representation theory and D-modules on flag varieties, Astérisque 173-174, Orbites Unipotentes et Représentations, Soc. Math. France (1989) 55-109.

[18] _ Equivariant derived categories and representations of real semisimple Lie groups in "Representation Theory and Complex Analysis", to appear in Lecture Notes in Mathematics, Springer Verlag.

[19] _ D-modules and microlocal calculus, Translated from the 2000 Japanese original by Mutsumi Saito, Translations of Mathematical Monographs 217, Iwanami Series in Modern Mathematics, American Mathematical Society, Providence, RI, 2003.

[20] Kashiwara, Masaki and Kawai, Takahiro, On holonomic systems of microdifferential equations. III. Systems with regular singularities, Publ. Res. Inst. Math. Sci. 17 (1981), no. 3, 813-979.

[21] Kazhdan, David, Kostant, Bertram and Sternberg, Shlomo, Hamiltonian group actions and dynamical systems of Calogero type, Comm. Pure Appl. Math. 31 (1978), no. 4, 481-507.

[22] Kontsevich, Maxim, Deformation quantization of algebraic varieties, Lett. Math. Phys. 56 (2001), no. 3, 271-294. 
[23] Nakajima, Hiraku, Lectures on Hilbert schemes of points on surfaces, University Lecture Series, 18, American Mathematical Society, Providence, RI, 1999.

[24] Polesello, Pietro and Schapira, Pierre, Stacks of quantization-deformation modules on complex symplectic manifolds, Int. Math. Res. Not. 2004, no. 49, 2637-2664.

[25] Schapira, Pierre, Microdifferential systems in the complex domain, Grundlehren der Mathematischen Wissenschaften, 269, Springer-Verlag, Berlin, 1985.

M.K.: Research Institute for Mathematical Sciences, Kyoto University, Kyoto 606, JAPAN

R.R.: Mathematical Institute, University of Oxford, 24-29 St Giles', Oxford, OX1 3LB, UK 